This PDF is a selection from a published volume from the National Bureau of Economic Research

Volume Title: NBER International Seminar on Macroeconomics 2006

Volume Author/Editor: Lucrezia Reichlin and Kenneth West, organizers

Volume Publisher: University of Chicago Press

Volume ISBN: 978-0-226-70742-6

Volume URL: http://www.nber.org/books/reic08-1

Conference Date: June 16-17, 2006

Publication Date: July 2008

Title: Pension Systems and the Allocation of Macroeconomic Risk

Author: Lans Bovenberg, Harald Uhlig

URL: http://www.nber.org/chapters/c7038 


\title{
Pension Systems and the Allocation of Macroeconomic Risk
}

\author{
Lans Bovenberg, CentER, Tilburg University, and CEPR \\ Harald Uhlig, Humboldt University Berlin, Deutsche Bundesbank, \\ CentER, and CEPR
}

\subsection{Introduction}

One can distinguish pension systems along three dimensions (see Lindbeck and Persson [2003]): defined benefit versus defined contribution, funded versus pay as you go, and actuarial versus nonactuarial. The first dimension involves intergenerational risk sharing: how are macroeconomic risks distributed over various generations? In particular, in defined-benefit systems, the retired generations are shielded from macroeconomic risks. In defined-contribution systems, in contrast, these generations are bearing some of the investment risk. The second dimension relates to intergenerational redistribution: are there predictable transfers from the young, working generations to the retired generations? The third dimension, finally, involves the link between contributions and benefits on an individual level. A system is actuarially fair if the individual premium paid corresponds to the actuarial value of the additional pension benefits that are being accumulated.

This paper explores the first two dimensions of an optimal pension system. In exploring optimal pension systems, we distinguish between predictable intergenerational transfers and optimal risk sharing. Hence, in contrast to Merton (1983) and Krueger and Kubler $(2002,2005)$, we allow the government to enhance intergenerational risk sharing without necessarily implementing a pay-as-you-go system. Indeed, defined benefit-funded pension systems that link benefits to wage rates and absorb financial market shocks by adjusting pension premia paid by the young can help to trade risks between the young, who are long on human capital and the older generation, who are long on financial capital. In contrast to defined-benefit pay-as-you-go systems, these funded systems enhance risk sharing without crowding out capital formation. We analyze the optimal mix between defined-benefit and defined-contribution 
pensions as part of the optimal risk-sharing scheme, while at the same time determining the optimal mix between funded and pay-as-you-go financing as part of the optimal redistribution between old, young, and future generations.

To investigate optimal intergenerational redistribution and optimal intergenerational risk sharing in a general equilibrium setting, we develop a stochastic overlapping-generations model of a closed economy featuring endogenous growth. Optimal risk sharing under an ex ante welfare criterion has been explored in a partial-equilibrium setting by Gordon and Varian (1988), Shiller (1999), and De Menil, Murtin, and Sheshinski (2005). Our analysis builds on the general equilibrium model of Bohn (1998, 1999, 2003, 2005), who investigates optimal risk sharing under an ex ante welfare criterion between overlapping generations in an exogenous growth model with capital accumulation. Regarding related contributions on this issue, see also Olovsson (2004) for optimal risk sharing under an ex post criterion, see Blanchard and Weil (2001), Demange (2002), and Barbie, Hagedorn, and $\operatorname{Kaul}(2003,2004)$, while for the role of agent heterogeneity, see, for example, Conesa and Krueger (1999). We extend Bohn's analysis by incorporating endogenous growth so that we can also explore the optimal response of long-term growth to various productivity and demographic shocks. Moreover, we integrate various elements that Bohn has studied in separate papers, such as endogenous labor supply (see Bohn [1998]), productivity shocks (in labor productivity, factor productivity, and depreciation, see Bohn [1998]) and demographic shocks (fertility and longevity, see Bohn [1999]). We also explore how government transfers between generations should optimally respond to various shocks and clearly distinguish between predictable intergenerational transfers and optimal risk sharing.

Key to studying issues of risk sharing is the issue of risk aversion. For that reason, we pay particular attention to the distinction between intertemporal substitution and risk aversion as formulated by Epstein and Zin (1989) or Weil (1990). In fact, as in Bohn (1998), we also allow for risk aversion of the young agents, which one may either read as a desire of the social planner toward insuring the yet-unborn, or life-risk aversion of young agents before they are able to participate in market activities. The life-risk aversion parameter will play a crucial role.

As in the classic overlapping-generations model of Diamond (1965), at any point in time two generations are alive. Only the older generation participates in the capital market and is thus subject to capital-market risks. The younger generation works and is subject to labor-income 
risks. These two overlapping generations cannot trade risks in the capital market because the young cannot participate in the capital market before the shocks occur and thus cannot insure against the realization of uncertainty at their birth. An alternative interpretation is that the young cannot borrow against their human capital to invest in the capital market. The young, who are long on human capital, and the old, who are long on physical capital, thus have to rely on government intervention to trade and diversify risks. To illustrate: through the pension system, the government, in effect can create new, nontradable assets that are not traded in financial markets and give the old a claim on labor income and the young a claim on capital income. By endowing the various generations with net positions in these assets, the government can, in principle, create an insurance equilibrium that would emerge if agents could freely trade ex ante. The government can trade not only risks between these two overlapping generations but also shift risks between current generations and future generations through capital accumulation. In this way, the government can also engineer implicit trades between nonoverlapping generations. We thus consider risk sharing both within periods between overlapping generations and across time between nonoverlapping generations.

We consider a social planning problem and characterize some necessary properties of optimal risk sharing arrangements across generations. Our economy features three imperfections calling for government intervention. First, the endogenous growth feature implies an externality in capital formation, which calls for an investment subsidy. Second, the intergenerational distribution may not be optimal from the point of view of a social planner, who thus may want to engage in intergenerational redistribution. Third, ex ante trading in risks between generations is not possible because generations can participate in capital markets only after they are born and most shocks have already materialized. Shifting risks to the groups who can best bear them can thus create an ex ante Pareto improvement and maintains incentives for risk-taking in general equilibrium. Improved risk sharing generates a Pareto improvement only in an ex ante, not an ex post sense. Indeed, thinking about risk sharing before shocks actually hit helps to avoid divisive battles about intergenerational redistribution after the shocks have in fact materialized.

The rest of the paper is structured as follows. After section 5.2 formulates the model, section 5.3 sets up the social planners' problem and interprets the conditions for optimal intergenerational risk sharing. Sec- 
tion 5.4 solves the social planners' problem. It investigates the solution to the steady state, showing the calculation of the equilibrium boils down to solving one equation in labor supply. For the dynamics, it relies on using log-linearization techniques rather than projection algorithms, as in Krueger and Kubler (2004), in order to derive insights into the linearized behavior around the steady state. In particular, it is shown that the dynamics of the model are characterized by a single endogenousstate vector, which combines ex ante utilities as well as the current capital stock. We thus provide a flexible framework that can be used and calibrated as a workhorse for exploring intergenerational risk sharing with endogenous growth. Section 5.5 explores how the social optimum can be decentralized through a pension system. Section 5.6 provides some quantitative explorations of the model and investigates the factors determining optimal intergenerational distribution and risk sharing. Section 5.7 concludes.

We obtain the following key results.

1. We provide a general characterization of the optimal risk-sharing condition; see, in particular, propositions 1,2, and 3. A detailed interpretation is provided subsequent to proposition 3 .

2. An endogenous growth model is provided, which is fairly tractable, despite featuring endogenous labor supply, a number of different shocks, and nonseparable preferences. In passing, we provide the conditions necessary for balanced growth under Epstein-Zin preferences (see section 5.3.2 and appendix A.2). In special cases, it is possible to characterize the steady state completely, while it generally requires solving a one-dimensional equation characterizing steady-state labor supply (see section 4 and in particular equation [62] and appendices A.4.5 and A.4.7).

3. We use log-linearization techniques to characterize the response to shocks (see propositions 2 and 3 as well as appendices 5.A.1 and 5.A.4.8), including implications for risk premia (see Appendix 5.A.3). It should be understood that this is a small-shock approximation, or an approximation to shocks with bounded support (see Samuelson [1970] and the discussion in Judd and Guu [2001]). Despite the limitations of loglinear approximations, this provides a useful starting point for further explorations of risk sharing with higher-order numerical techniquesor, alternatively, for searching for interesting special cases, which can be solved analytically. 
4. The endogenous dynamics can be shown to solve a third-degree polynomial in a single-state variable, with typically only one stationary solution. This is explicitly shown in Appendix 5.A.5. Thus, the model is suitable for exploring a rich set of features while keeping the amount of analytics moderate, providing a starting point and intuition for models featuring a larger number of periods of life.

5. A number of interesting insights emerge from the quantitative exploration of the model (see section 5.6). The usual caveat, that these statements are strictly valid only for the parameter ranges investigated (and should not be read as globally valid theorems) applies.

a. At a benchmark parameterization, the old bear a smaller burden of the risk in productivity surprises, if old-age risk aversion is larger than life risk aversion, but a larger one, if the old-age risk aversion is smaller (see equation [53] and section 5.6.5). Thus, whether young agents should insure the old agents against the random returns on stock markets due to the uncertainty in technological progress depends on how one views the risk aversion of old people versus the desire to insure the yet-unborn against their life risk. Note how a fully funded defined-contribution system, where the old save for retirement by holding equity, would lead to an equal sharing of the productivity risk between young and old in the case of full depreciation, while a defined-benefit system would impose the entire risk on the young. Neither solution is typically optimal from a social planner's perspective, although the defined-contribution private capital system seems to be optimal in some special circumstances with respect to productivity shocks that equally affect labor and capital income.

b. Consumption of the young and the old always moves in the same direction, even for a population growth shock, where the size of the new young generation is larger than expected (see section 5.6.6). This result is in contrast to the result of a fully funded decentralized system without risk sharing between generations: there, the old would receive higher consumption (due to the increase in return to capital), while the young would receive lower consumption (due to decreasing marginal returns in labor from the larger working population).

c. Persistent increases in longevity will lead to lower total consumption of the old (and thus certainly lower per-period consumption of the old) and the young as well as higher work effort of the young. The additional resources are used to increase growth and future output, 
resulting in higher consumption of future generations (see section 5.6.7). Thus, increases in life expectancy require old agents not only to get by with less per period but also with less in total, in contrast to what a defined-benefit system or standard insurance contracts might offer.

\subsection{The Model}

\subsubsection{Motivation and Overview}

We provide a model in which the key features can be studied while remaining tractable. As a starting point, we follow Yanagawa and Uhlig (1996) and construct an overlapping-generations model with capital and endogenous growth, effectively delivering an AK model. This eliminates capital as a state variable, thus simplifying the analysis. Since we want to study both risk aversion and intertemporal substitution, we keep them separate, using Epstein-Zin preferences. As sources of risks, we allow for variations in factor productivities, depreciation, longevity, and population growth.

\subsubsection{The Environment}

Consider a discrete time overlapping-generations model with $t=1,2$, ... in which all agents live for two periods and where random shocks occur at the beginning of each period. For convenience, tables 5.1 and 5.2 summarize the symbols. Let $c_{t, y}, c_{t+1, o}$ denote the per capita consumption of the representative agent born at the beginning of period $t$ when young and when old, respectively. Agents work only during the first period of their lives. The labor supply of the generation born in period $t$ is denoted by $n_{t}$. The fraction of the second period during which the old are actually alive to enjoy consumption is denoted by $\varpi_{t+1}$. An exogenous variable, $\boldsymbol{\omega}_{t+1}$, is known at the beginning of period $t+1$. It measures the remaining expected lifetime of the old, given the medical and demographic knowledge at the beginning of period $t+1$. We define $c_{t+1,0}$ as total consumption of the old in the second period, so that $c_{t+1,0} /$ $\varpi_{t+1}$ represents consumption of the old per unit of time. Given some utility $q(\cdot)$ for old-age consumption per unit of time, total utility when old is

$$
U_{t, t+1}=\varpi_{t+1} q\left(\frac{c_{t+1,0}}{\varpi_{t+1}}\right) \text {. }
$$


Table 5.1.

Summary table for the notation and symbols used in this paper, part 1.

\begin{tabular}{|c|c|c|}
\hline Notation & Meaning & First occurance \\
\hline$A_{t}$ & total factor productivity & (7) \\
\hline$a_{t}$ & argument of $z(\cdot)$. & (28) \\
\hline$C_{l, y}$ & aggregate cons. of young at $t$ & (9) \\
\hline$c_{t, y}$ & cons. of gen. born in $t$, when young & (2) \\
\hline$\tilde{c}_{t, y}$ & rescaled consumption & below definition 5 \\
\hline$C_{t, 0}$ & aggregate cons. of old at $t$ & (9) \\
\hline$c_{t+1,0}$ & cons. of gen. born in $t$, when old & (2) \\
\hline$\tilde{\boldsymbol{c}}_{t, 0}$ & rescaled consumption & below definition 5 \\
\hline$d_{t}$ & discounting correction & $(20)$ \\
\hline$f(\cdot)$ & production function & (7) \\
\hline $\mathscr{H}_{1}$ & set of all possib. histories at $t$ & below (11) \\
\hline $\mathscr{H}$ & set of all possible histories & below (11) \\
\hline$h_{t}$ & history up to $t$ & (11) \\
\hline$K_{t-1}$ & aggr. capital, used in prod. at $t$ & (7) \\
\hline$k_{t-1}$ & capital in prod. at $t$, per old & $(8)$ \\
\hline$\ell_{z, c, t}$ & logarithm. deriv. of $u$ w.r.t. $c$ & above (31) \\
\hline$\ell_{u, n, t}$ & logarithm. deriv. of $u$ w.r.t. $n$ & above (31) \\
\hline$\ell_{x, t}$ & logarithm. deriv. of $x$ & above (31) \\
\hline$\ell_{q, t}$ & logarithm. deriv. of $q$ & above (31) \\
\hline$\ell_{v}$ & logarithm. deriv. of $v$ & $(43)$ \\
\hline$m_{t+1}$ & discount factor of soc. plan. & (23) \\
\hline$n_{t}$ & labor supply of gen. born in $t$, when young & $(2)$ \\
\hline$q(\cdot)$ & utility function when old & $(2)$ \\
\hline$R_{r}$ & social rate of return & $(24)$ \\
\hline $\mathscr{S}$ & state space for date-t-variables & below $(10)$ \\
\hline $\mathbf{S R}_{t}$ & Sharpe ratio & (98) \\
\hline$U(\cdot)$ & overall utility & (2) \\
\hline$U_{t, t}$ & overall utility of generation $t$, cond. on $t$-info & $(2)$ \\
\hline$u(\cdot, \cdot)$ & instantaneous utility when young & $(2)$ \\
\hline$u_{0}$ & parameter for initially old & (5) \\
\hline$U_{t}\left(h_{t}\right)$ & ex post utility & below definition 1 \\
\hline$U_{t, 0}$ & ex ante expected utility at beginning of time & (12) \\
\hline$x(\cdot)$ & risk-aversion transformation & $(2)$ \\
\hline$Y$, & aggregate output at $t$ & (7) \\
\hline$y_{t}$ & output at $t$, per old & $(8)$ \\
\hline$z(\cdot)$ & overall ex ante risk-aversion function & $(2)$ \\
\hline$Z_{t}$ & labor-specific productivity $t$ & (7) \\
\hline
\end{tabular}


Table 5.2.

Summary table for the notation and symbols used in this paper, part 2.

\begin{tabular}{|c|c|c|}
\hline Notation & Meaning & First occurance \\
\hline$\alpha_{1}$ & argument share & above (31) \\
\hline$\beta$ & discount factor of agent & $(2)$ \\
\hline$x$ & pref. parameter for leisure & $(55)$ \\
\hline$\delta_{t}$ & depreciation rate & (9) \\
\hline$\mu_{z, i}$ & (negative) elasticity of $z^{\prime}(\cdot)$ & above (31) \\
\hline$\mu_{H e, c, t}$ & (negative) elasticity of $u_{c}(\cdot$,$) w.r.t. c$ & above (31) \\
\hline$\mu_{u t, n, t, t}$ & (negative) elasticity of $u_{c}(\cdot$,$) w.r.t. n$ & above (31) \\
\hline$\mu_{x, i}$ & (negative) elasticity of $x^{\prime}(\cdot)$ & above (31) \\
\hline$\mu_{t, t, t}$ & (negative) elasticity of $q^{\prime}(\cdot)$ & above (31) \\
\hline$\phi$ & scale factor & in definition 5 \\
\hline$\Phi(\cdot)$ & allocation & in definition 1 \\
\hline$\ddot{\Phi}$ & rescaled allocation & in definition 5 \\
\hline$\gamma_{t}$ & extra (policy) shock & below $(10)$ \\
\hline$\eta$ & inverse of intertemp. elast. of subst. & above (40), (42) \\
\hline$\iota$ & $x^{-1}$ & just below (78) \\
\hline$\kappa\left(n_{f}\right)$ & integration constant & $(40)$ \\
\hline к & integration constant & below $(40)$ \\
\hline$\lambda_{t}$ & Lagrange multiplicator on feasibility & (14) \\
\hline$v$ & risk avers. w.r.t. old-age risk & $(44)$ \\
\hline$\Pi_{t}$ & young population & (6) \\
\hline$\pi$ & young population growth factor & (6) \\
\hline$\sigma_{y}$ & income share & $(88)$ \\
\hline$\sigma_{t}$ & return subsidy & $(69)$ \\
\hline$\tau_{t, y}$ & lump-sum tax on young & $(68)$ \\
\hline$\tau_{t, o}$ & lump-sum tax on old & $(69)$ \\
\hline$\tau_{t \text {,priv }}$ & tax, if priv. cap. & $(70)$ \\
\hline$\tau_{t \text {,pub }}$ & tax, if publ. cap. & $(71)$ \\
\hline$\xi$ & life time risk-aversion parameter & $(44)$ \\
\hline$\omega_{i}$ & welfare weight & in definition 4 \\
\hline$\omega$ & welfare weight factor & below definition 4 \\
\hline$\rho$ & correlation & before (98) \\
\hline$\psi$ & production function param. & $(56)$ \\
\hline$\theta$ & production function param. & $(56)$ \\
\hline w, & expected lifetime of the old in period $t$ & $(2)$ \\
\hline s & exponent of $w$ & $(102)$ \\
\hline$\zeta$ & vector of exog. var. & $(103)$ \\
\hline
\end{tabular}


We wish to distinguish intertemporal elasticity of substitution from risk aversion. We therefore use some concave and differentiable function $x(\cdot)$ to increase the curvature of $U_{t, i+1}$ before taking expectations and undoing this curvature again. Furthermore, we allow for risk aversion to lifetime risk (or, alternatively, for a preference of the social planner toward equality across generations). Overall preferences of the generation born at the beginning of time $t$ (conditional on the information that is available during that period) are assumed to be given by

$$
\begin{aligned}
U_{t, t} & =U\left(c_{t, y^{\prime}} n_{t}, c_{t+1, o} ; \boldsymbol{\varpi}_{t+1}\right) \\
& =z\left[u\left(c_{t, y}, n_{t}\right)+\beta x^{-1}\left\{E_{t}\left[x\left(U_{t, t+1}\right)\right]\right\}\right] \\
& =z\left[u\left(c_{t, y^{\prime}} n_{t}\right)+\beta x^{-1}\left(E_{t}\left\{x\left[\varpi_{t+1} q\left(\frac{c_{t+1, o}}{\varpi_{t+1}}\right)\right]\right\}\right)\right],
\end{aligned}
$$

where $u(\cdot$,$) is an instantaneous utility function in consumption and$ work effort during the first period of life, and $z(\cdot), x(\cdot)$, and $q(\cdot)$ are strictly increasing and continuously differentiable functions such that $U(\cdot)$ is strictly concave in $c_{t, y}, n_{t}$ and $c_{t+1,0}$.

As an important special case, consider

$$
U_{t, t}=\frac{\left(\left[c_{t, y} v(n)\right]^{1-\eta}+\beta\left\{E_{t}\left[\left(\varpi_{t+1}^{\eta} c_{t+1, o}^{1-\eta}\right)^{(1-v) /(1-\eta)}\right]\right\}^{(1-\eta) /(1-v)}\right)^{(1-\xi) /(1-\eta)}}{1-\xi} .
$$

where $\xi \geq 0$ and $v \geq \eta \geq 0$ (see also equation [44]). This specification and its consequences are discussed in greater detail in section 5.3.3.

For the generation that is old at the beginning of the planning period, $U_{0,1}$ are the preferences at the start of period 1, (see equation [1]), and

$$
\begin{aligned}
U_{0,0} & =z\left(u_{0}+\beta x^{-1}\left\{E_{0}\left[x\left(U_{0,1}\right)\right]\right\}\right) \\
& =z\left[u_{0}+\beta x^{-1}\left(E_{0}\left\{x\left[\varpi_{1} q\left(\frac{c_{1, o}}{\varpi_{1}}\right)\right]\right\}\right)\right],
\end{aligned}
$$

for some parameter $u_{0}$ are the preferences of the initially old, when considering them ex ante, that is, behind the veil of ignorance before the uncertainty about the first period is resolved. We have incorporated $z(\cdot)$ and $u_{0}$ as a normalization in order to compare utilities of the initially old and agents living in two periods in the same units, when studying the social planner's problem.

Let the population of the young at date $t$ be $\Pi_{t}$, and denote the growth factor of the young population as 
$\pi_{t}=\frac{\Pi_{t}}{\Pi_{t-1}}$.

We use capital letters to denote aggregate variables, so that $C_{t, y}=$ $\Pi_{t} c_{t, y}$, and so on. With $k_{t-1}$, we denote the capital stock available for production per old person at date $t$. Thus, $K_{t-1}=\Pi_{t-1} k_{t-1}$ represents the aggregate capital stock in period $t$.

The aggregate production function is given by

$Y_{t}=A_{t} K_{t-1} f\left(Z_{t} n_{t}\right)$

for some positive, concave, strictly increasing, and strictly concave function $f(\cdot)$. Here, $A$, stands for a total factor productivity parameter and $Z_{t}$ represents a labor-specific productivity parameter. Aggregate production $Y_{f}$ is proportional to the aggregate capital stock. We are thus essentially assuming an AK model. Note that labor per young person, $n_{t}$, rather than aggregate labor, $\Pi_{t} n_{t}$, appears as an argument of the function $f(\cdot)$. This aggregate-AK production function can arise from a decentralized production economy with a production externality that is proportional to the capital stock per young person, $K_{t-1} / \Pi_{t}$ (see section 5.5).

With $y_{t}$, we denote aggregate production, divided by the population of the old (i.e., $y_{t}=Y_{t} / \Pi_{t-1}$ )

$y_{t}=A_{t} k_{t-1} f\left(Z_{t} n_{t}\right)$.

Aggregate feasibility requires that

$C_{t, y}+C_{i, 0}+K_{t}=Y_{i}+\left(1-\delta_{i}\right) K_{t-1}$

where we allow for time variation in the depreciation rate $\delta_{t}$. Expressed relative to the older population, this equation reads (after substituting [8] to eliminate $y_{t}$ )

$c_{t, y} \pi_{t}+c_{t, 0}+k_{t} \pi_{t}=\left(A_{t} f\left(Z_{t} n_{t}\right)+1-\delta_{t}\right) k_{t-1}$.

The vector $\left(Z_{t}, A_{t}, \delta_{t}, \pi_{t}, \varpi_{t}\right) \in \mathscr{Y}$ is stochastic and i.i.d., and where $\mathscr{S}$ $=\Re_{++}^{5} ; k_{0}$ and $\Pi_{0}$ are given and nonrandom, and we normalize $\Pi_{0}=1$.

$h_{t}=\left[\left(Z_{t}, A_{t}, \delta_{t}, \pi_{t}, \varpi_{t}\right), \ldots,\left(Z_{1}, A_{1}, \delta_{1}, \pi_{1}, \varpi_{1}\right)\right]$

denotes the history up to and including $t$. Let $\mathscr{H}_{t}=\left(h_{t} \mid\right.$ all $\left.h_{t}\right)=\mathscr{S}^{t} \subseteq \mathfrak{R}^{5 t}$ denote the set of all histories up to and including $t$ and let $\mathscr{H}=\cup_{t=1}^{\infty} \mathscr{H}_{t}$ denote their union.

Let some initial level of capital $k_{-1}^{*}$ be given. 
Definition 1 A feasible allocation is a mapping from the set of all histories into a vector of positive real numbers, $\Phi: \mathscr{H} \rightarrow \Re_{+}^{5}$ such that $k_{-1}=k_{-1}^{*}$ and the vector $\left(c_{t, y}, c_{t, o}, n_{t}, k_{t}\right)=\Phi\left(h_{t}\right)$ satisfies (10) for all histories $h_{t} \in \mathscr{H}$.

Associated with a feasible allocation are ex post utilities $U_{t, t}=U_{t}\left(h_{t}\right)$, $U_{0,1}=U_{0}\left(h_{1}\right)$.

\subsection{The Social Planner's Problem}

The social planner maximizes the utility of the agents (2) subject to the feasibility constraint $(10)$ given the exogenous stochastic process $\left(Z_{t}, A_{t}\right.$, $\left.\delta_{t}, \pi_{t}, \varpi_{t}\right)$. In this connection, one needs to take a stand on whether agents born at the same time but with different histories are different agents or not. If they are different, then insurance of young agents against shocks during their period of birth (i.e., redistributing from young agents born in good times to young agents born in bad times) will typically not constitute a Pareto improvement. Indeed, this would be a redistribution from good-state agents to bad-state agents.

Definition 2 [PO 1] A noninsurance Pareto optimum is a feasible allocation with associated ex post utilities $U_{t, k}$ (i.e., [2]) and $U_{0,1}$ see [1], such that no other feasible allocation exists that attains equally large utilities for all histories and generations and a strictly larger utility for at least one history and generation.

When contemplating insurance, the social planner compares states with different population sizes. In particular, the social planner treats all agents born at the same date and under the same history in the same way and thus assigns the same weight per person across different states of nature. Hence, the ex ante preferences of the social planner for generation $t>0$ at the beginning of time amount to

$U_{t, 0}=E_{0}\left(\Pi_{t} U_{t, t}\right)$.

The ex ante preferences of the social planner for the initial old are given by $U_{0,0}$, stated in (5) in view of our normalization $\Pi_{0}=1$.

Definition 3 [PO 2] A Pareto optimum with insurance is a feasible allocation with associated ex ante utilities $U_{0.0}$ (see [5] and $U_{t, 0}[12]$ ), such that no other feasible allocation exists attaining equally large ex ante utilities for all generations and strictly larger ex ante utility for at least one generation. 
Clearly, any Pareto optimum with insurance is also a noninsurance Pareto optimum, but not vice versa. As an example, suppose that in some allocation $A$, a particular generation receives consumption $c-\mu$ if times are bad and $c+\mu$ if times are good, for some $\mu>0$. Suppose that bad times and good times are equally likely. Suppose finally that some alternative allocation $B$ exists, equivalent in all aspects, except that this generation then receives consumption $c$ in both eventualities. In view of the concavity of the preferences, allocation $A$ could not be a Pareto optimum with insurance, since allocation $B$ provides insurance ex ante. However, allocation $A$ might well be a noninsurance Pareto optimum, since the generation born in good times is considered to be different from the generation born in bad times. Thus, conditions necessary for a noninsurance Pareto op timum are also necessary for a Pareto op timum with insurance. Voluntary schemes can implement noninsurance Pareto optima (see, e.g., Blanchard and Weil [2001] and Barbie, Hagedorn, and Kaul [2003, 2004]). Insurance optima, in contrast, require compulsory participation to commit generations to the ex post redistribution that is implied by ex ante insurance.

Pareto optima with insurance are more interesting for our purposes because the issue of intergenerational risk sharing among the yet unborn is at the heart of our analysis. One should bear in mind, however, that our analysis involves a choice regarding the welfare weights assigned to agents of the same period born under different histories: their relative weights correspond to the relative probabilities of their specific histories.

To provide a single objective for the social planner, we follow Bohn (2003) in formulating a weighted sum of the ex ante utilities of the various generations, with $\left(\omega_{t}\right)_{t=0}^{\infty}$ as a sequence of (nonstochastic) welfare weights.

Definition 4 (SP) A social optimum for welfare weights $\left(\omega_{t}\right)_{t=0}^{\infty}$ is a feasible allocation solving

$$
\max _{(\Phi \mid \Phi \text { feas. alloc. })} \sum_{t=0}^{\infty} \omega_{t} U_{t, 0}
$$

This social optimum implements a particular Pareto optimum with insurance. Using equation (12), we can write the social objective function as

$$
\max _{(\Phi|\Phi| \Phi \text { feas. alloc) })}\left[\omega_{0} U_{0,0}+E_{0}\left(\sum_{t=1}^{\infty} \omega_{t} \Pi_{t} U_{t, t}\right)\right] .
$$


To focus on stationary solutions, we implement exponential discounting so that $\omega_{t}=\omega^{t}$.

\subsubsection{First-order Conditions}

Consider the problem of finding a social optimum for welfare weights $\omega_{t}=\omega^{t}$. Let $\omega^{t} \lambda_{t}$ be the Lagrange multiplier on the aggregate feasibility equation (9) so that $\Pi_{t-1} \omega^{t} \lambda_{t}$ is the Lagrange multiplier on the feasibility constraint (10). For ease of notation, assume that random variables can only take one of finitely many values, each with positive probability given any history. Let $\mathscr{H}_{t}$ be the set of all histories up to and including $t$, and let $\operatorname{Prob}\left(h_{t}\right)$ be its unconditional probability. The Lagrangian then amounts to

$$
\begin{gathered}
\max _{(\Phi \mid \Phi \text { feas. alloc. })}\left[\omega_{0} U_{0,0}+\sum_{t=1}^{\infty} \sum_{h_{t} \in \mathscr{t}_{t}} \operatorname{Prob}\left(h_{t}\right) \boldsymbol{\omega}^{t}\left(\Pi_{t} U_{t, t}\left(h_{t}\right)-\lambda_{t}\left(h_{t}\right) \Pi_{t-1}\right.\right. \\
\left.\cdot\left\{c_{t, y} \pi_{t}+c_{t, o}+k_{t} \pi_{t}-\left[A_{t} f\left(Z_{t} n_{t}\right)+1-\delta_{t}\right] k_{t-1}\right\}\right],
\end{gathered}
$$

We have made the dependence of only $\lambda_{t}$ and $U_{t, t}$ on $h_{t}$ explicit. In fact, the population, their growth rates, and all economic choices at date $t$, like $c_{t, y}$ or $k_{t}$, are similarly functions of $h_{t}$.

Dropping the argument $h_{t}$, the first-order conditions are

$$
\begin{aligned}
& \left(\frac{\partial}{\partial c_{t, y}}:\right) \quad \lambda_{t}=\frac{\partial U_{t, t}}{\partial c_{t, y}}, \\
& \left(\frac{\partial}{\partial c_{t, 0}}:\right) \quad \lambda_{t} \operatorname{Prob}\left(h_{t} \mid h_{t-1}\right)=\frac{1}{\omega} \frac{\partial U_{t-1, t-1}}{\partial c_{t, o}}, \\
& \left(\frac{\partial}{\partial n_{t}}:\right) \quad \lambda_{t} A_{t} k_{t-1} f^{\prime}\left(Z_{t} n_{t}\right) Z_{t}=-\pi_{t} \frac{\partial U_{t, t}}{\partial n_{t}}, \\
& \left(\frac{\partial}{\partial k_{t}}:\right) \quad \lambda_{t}=\omega E_{t}\left\{\lambda_{t+1}\left[A_{t+1} f\left(Z_{t+1} n_{t+1}\right)+1-\delta_{t+1}\right]\right\} .
\end{aligned}
$$

In equation (16) we have been a bit more formal than usual. A more general treatment would involve treating this equation as an equality between measures or their Radon-Nikodym derivatives. The right-hand side of that equation involves taking the derivative of a conditional expectation with respect to one of its arguments. To be more explicit (and 
after cancellations of the conditional probability terms on both sides of the equation), (16) can be rewritten as

$\lambda_{t}=\frac{\beta}{\omega} d_{t} q^{\prime}\left(\frac{c_{t, o}}{\varpi_{t}}\right)$

where

$d_{t}=z^{\prime}\left(a_{t-1}\right)\left(x^{-1}\right)^{\prime}\left(E_{t-1}\left\{x\left[\varpi_{t} q\left(\frac{c_{t, o}}{\varpi_{t}}\right)\right]\right\}\right) x^{\prime}\left[\varpi_{t} q\left(\frac{c_{t, o}}{\varpi_{t}}\right)\right]$

is a discounting correction due to the separation of intertemporal substitution and risk aversion as well as allowing for life-risk aversion: for the definition of $z^{\prime}\left(a_{t-1}\right)$, see equation (28).

Define $w_{t}$ to be the marginal social productivity per unit of labor:

$w_{t} \equiv A_{t} k_{t-1} f^{\prime}\left(Z_{t} n_{t}\right) \frac{Z_{t}}{\pi_{t}}$.

The first and third first-order condition together imply

$\frac{\partial u\left(c_{t, y^{\prime}} n_{t}\right)}{\partial c_{t, y}} w_{t}=-\frac{\partial u\left(c_{t, y^{\prime}} n_{t}\right)}{\partial n_{t}}$

which is the familiar condition that the marginal rate of substitution between consumption and leisure should equal its social opportunity costs, that is, the aggregate marginal rate of transformation.

Define the stochastic discount factor of the social planner as

$m_{t+1}=\frac{\omega \lambda_{t+1}}{\lambda_{t}}$

and the social rate of return as

$R_{t}=A_{t} f\left(Z_{t} n_{t}\right)+1-\delta_{t}$.

We can then write the fourth first-order condition as a familiar assetprice equation

$1=E_{t}\left(m_{t+1} R_{t+1}\right)$.

Substitute $\lambda_{t}$ from (15), $\lambda_{t+1}$ from (19), and $A_{t} f\left(Z_{t} n_{t}\right)+1-\delta_{t}$ from (24) into (18) to obtain

$z^{\prime}\left(a_{t}\right) \frac{\partial u}{\partial c_{t, y}}=\beta E_{t}\left[d_{t+1} q^{\prime}\left(\frac{c_{t+1, o}}{\varpi_{t+1}}\right) R_{t+1}\right]$

or explicitly 


$$
\begin{aligned}
\frac{\partial u\left(c_{t, y^{\prime}} n_{t}\right)}{\partial c_{t, y}}= & \beta\left(x^{-1}\right)^{\prime}\left(E_{t}\left\{x\left[\varpi_{t+1} q\left(\frac{c_{t+1, o}}{\varpi_{t+1}}\right)\right]\right\}\right) \\
& \cdot E_{t}\left\{x^{\prime}\left[\varpi_{t+1} q\left(\frac{c_{t+1, o}}{\varpi_{t+1}}\right)\right] q^{\prime}\left(\frac{c_{t+1, o}}{\varpi_{t+1}}\right) R_{t+1}\right\} .
\end{aligned}
$$

Equation (25) is the familiar asset-pricing equation in terms of marginal utilities of generation $t$, adjusted with the discounting-correction term $d_{t+1}$ and longevity risk $\varpi_{t+1}$.

\subsubsection{Risk Sharing}

The first two first-order conditions imply

$\lambda_{t} \operatorname{Prob}\left(h_{t} \mid h_{t-1}\right)=\frac{\partial U_{t, t}}{\partial c_{t, y}} \operatorname{Prob}\left(h_{t} \mid h_{t-1}\right)=\frac{1}{\omega} \frac{\partial U_{t-1, t-1}}{\partial c_{t, o}}$,

which is a risk-sharing or complete markets condition: young and old agents alive at the same time should evaluate risk using the same stochastic discount factor. Let $a_{t}$ be the argument of the $z(\cdot)$-function in the specification of the utility function (2), that is,

$a_{t}=u\left(c_{t, y}, n_{t}\right)+\beta x^{-1}\left(E_{t}\left\{x\left[\boldsymbol{\varpi}_{t+1} q\left(\frac{c_{t+1,0}}{\boldsymbol{\varpi}_{t+1}}\right)\right]\right\}\right)$

One can then write the risk-sharing condition (27) as follows.

PROPOSITION 1 [The risk-sharing condition.] At the social planner optimum with constant discounting of the welfare of future generations,

$z^{\prime}\left(a_{t}\right) \frac{\partial u\left(c_{t, y^{\prime}} n_{t}\right)}{\partial c_{t, y}}=z^{\prime}\left(a_{t-1}\right) \frac{\beta}{\omega} d_{t} q^{\prime}\left(\frac{c_{t, o}}{\boldsymbol{w}_{t}}\right)$

where $d_{t}$ is defined in (20) and $a_{t}$ is defined in equation (28).

ProOF. Direct.

In the special case of a linear $z(\cdot)$, the terms $z^{\prime}\left(a_{t}\right)$ and $z^{\prime}\left(a_{t-1}\right)$ drop out and one obtains the familiar condition, equating marginal utility of consumption for the young to a constant factor times marginal utility of consumption for the old state by state, and adjusted with the riskcurvature term.

In the general case, log linearization helps to deliver further insights. 
For the variables in (29), denote with an upper bar some benchmark value for each parameter, which we assume to be known as of date $t-1$ or possibly earlier, and which together satisfy equation (29). We use an inverted hat to denote the logarithmic deviation of a variable from this benchmark. Thus, for example,

$c_{t, y}=\bar{c}_{t, y} \exp \left(\check{c}_{t, y}\right)$

For any twice-differentiable function $y=f(x)$, define the logarithmic derivative

$\ell_{f}=\frac{f^{\prime}(x) x}{f(x)}=\frac{\partial \log (f\{\exp [\log (x)]\})}{\partial \log (x)}$

with the latter equation valid only if $x>0$ and $y>0$. Further, define the negative logarithmic derivative of its first derivative per

$\mu_{f}=-\frac{f^{\prime \prime}(x) x}{f^{\prime}(x)}$

For example, if $f(x)=c x^{\alpha}$, then $\ell_{f}=\alpha$ and $\mu_{f}=1-\alpha$. Often, $\mu_{f}$ arises as an elasticity.

We shall keep a subindex $t$ to denote the period for which the approximation applies. By assumption, the approximation is around a point known at $t-1$ or earlier. For functions with two arguments, we shall also note the argument(s) with respect to which the derivative is taken. For example, for some given utility function $u$, the intertemporal elasticity of substitution is given by the inverse of $\mu_{u c, c, t}$, whereas the cross-partial is given by

$\mu_{u c, n, t}=-\frac{u_{n c}\left(\bar{c}_{t, y}, \bar{n}_{t}\right) \bar{n}_{t}}{u_{c}\left(\bar{c}_{t, y}, \bar{n}_{t}\right)}$.

Furthermore, define the argument share

$\alpha_{t}=\frac{u\left(\bar{c}_{t, y}, \bar{n}_{i}\right)}{\bar{a}_{i}}$.

A complete, explicit list of all logarithmic derivatives can be found at the beginning of Appendix subsection 5.A.1.

PROPOSITION 2 [The loglinear risk-sharing condition.] Optimal intergenerational risk sharing implies the following first-order approximation to (29) in log deviations around the chosen benchmark, 
$\mu_{z, t} \check{a}_{t}+\mu_{u c, c, t} \check{t}_{t, y}+\mu_{u c, n, t} \check{n}_{t}=\mu_{z, t-1} \check{a}_{t-1}+\check{d}_{t}+\mu_{q, t}\left(\check{c}_{t, 0}-\check{\boldsymbol{\varpi}}_{t}\right)$

where

$\check{a}_{t}=\alpha_{t}\left(\ell_{u, c t} \check{t}_{t, y}+\ell_{u, n, t} \check{n}_{t}\right)+\left(1-\alpha_{t}\right)\left[\left(1-\ell_{q, t+1}\right) E_{t}\left(\check{\varpi}_{t+1}\right)+\ell_{q, t+1} E_{t}\left(\check{c}_{t+1,0}\right)\right]$

$\check{d}_{i}=\mu_{x, t}\left\{\left(1-\ell_{q, t}\right)\left[\check{\boldsymbol{\omega}}_{t}-E_{t-1}\left(\check{\boldsymbol{\omega}}_{t}\right)\right]+\ell_{q, t}\left[\check{c}_{t, o}-E_{t-1}\left(\check{c}_{t, o}\right)\right]\right\}$

PROOF. Calculate or follow the calculations in appendix A.1.

Consider the standard case, in which both $z(\cdot)$ and $x(\cdot)$ are linear, (so that $\mu_{x, t}=\mu_{z, t}=\mu_{z, t-1}=0$ ) and in which old and young agents feature the same risk aversion in consumption, that is, $\mu_{q, t}=\mu_{u c, c, t}$ ). If additionally, labor is assumed to be constant (i.e., $\check{n}_{t}=0$ ) or $u(c, n)$ is assumed to be separable in $c$ and $n$ (i.e., $\mu_{u c, n, t}=0$ ), one obtains

$\check{c}_{t, y}=\check{c}_{t, 0}-\check{\boldsymbol{\varpi}}_{t}$.

In that case, the percentage changes of the consumption of the young as well as of the old per unit of time should be exactly the same. One may want to consider this as a natural benchmark for risk sharing between young and old.

If labor is not assumed to be constant and $u(c, n)$ is not separable, equation (31) becomes instead

$\check{c}_{t, y}+\frac{\boldsymbol{\mu}_{u c, n, t}}{\mu_{u c, c, t}} \check{n}_{t}=\check{c}_{t, o}-\check{\boldsymbol{w}}_{t \prime}$

where one should note that $\mu_{u c, n, t}$ can have either sign. Consider the case where consumption and leisure are substitutes and hence, $\mu_{u c, n, t}<0$. If circumstances are such that the social planner commands more work from the young, $\check{n}_{i}>0$, the associated decline in leisure enjoyed by the young will make a marginal unit of consumption for the young relatively more valuable. In order to keep the total increase in marginal utility from consumption the same for both generations, the relative consumption decrease of the young should be smaller than the relative consumption decrease of the old per unit of time (i.e., $\check{c}_{t, y}>\check{c}_{t, 0}-\check{\varpi}_{t}$ ). The direction reverses if leisure and consumption are complements, $\mu_{u, t, n c}>$ 0 . Then, changes in leisure of the young (i.e., $-\check{n}_{t}$ ) are associated with changes in relative consumption of the young vis-à-vis the old, $\check{c}_{t, y}-\left(\check{c}_{t, o}\right.$ - $\check{\boldsymbol{\varpi}}_{t}$ ), which are of the same sign. With complementarity between leisure and consumption, changes in leisure are associated with changes in con- 
sumption that further increase the impact of leisure on the overall utility level of the young. Intuitively, decreases in leisure reduce the marginal utility of the young so that the young not only obtain less leisure but also consume less relative to the old.

Scale Invariance and Balanced Growth We wish to avoid effects from rescaling the units in which, for example, capital is measured. An alternative interpretation is that we seek a solution delivering a balanced growth path. Thus,

\section{Definition 5 Let the allocation}

$\Phi\left(h_{t}\right)=\left(c_{t, y}, c_{t, o}, n_{t}, y_{t}, k_{t}\right)$

be a solution to the social planner's problem, given initial capital $k_{-1}^{*}$. Let $\alpha_{t}\left(h_{t}\right)$ be the implied argument share (see equation [30]). The preference specification ${ }^{1}$ is said to be scale invariant if for all scalars $\phi>0, \tilde{\Phi}$ is a solution to the planner's problem with initial capital $\phi k_{-1}^{*}$, where

$\tilde{\Phi}\left(h_{t}\right)=\left(\phi c_{t, y}, \phi c_{t, o}, n_{t}, \phi y_{t}, \phi k_{t}\right)$

and if additionally the implied argument share $\tilde{\alpha}_{t}$ is unchanged (i.e., $\tilde{\alpha}_{t}\left[h_{t}\right]=$ $\left.\alpha_{t}\left[h_{l}\right]\right)$ for all histories $h_{l}$.

Scale invariance yields a number of implications (see the Appendix):

$$
\begin{aligned}
\frac{\ell_{u, c, t}}{\ell_{u, n, t}} & =-\frac{c_{t, y}}{w_{t} n_{t}}, \\
0 & =-\mu_{u c, c, t}+\mu_{u c, n, t} \frac{\ell_{u, c, t}}{\ell_{u, n, t}}+1, \\
\mu_{u c, c, t} & =\mu_{q, t+1} \\
\ell_{u, c, t} & =\ell_{q, t+1} .
\end{aligned}
$$

If in addition we impose that $\mu_{u c, c, l}$ is constant over time (i.e., $\eta \equiv \mu_{u c, c, t}$ ), we can derive a semiclosed form for the utility function $u(c, n)$, as is well known from the literature (see e.g., King, Plosser, and Rebelo [1988]):

$u\left(c_{t, y}, n_{t}\right)=\frac{\left[v\left(n_{t}\right) c_{t, y}\right]^{1-\eta}}{1-\eta}$

up to a constant, if $\eta \neq 1$ and 
$u\left(c_{t, y^{\prime}} n_{t}\right)=\log c_{t, y}+v\left(n_{t}\right)$

for $\eta=1$.

\subsubsection{Epstein-Zin Preferences}

We now provide a particular parametric example, satisfying the general implications listed in the scale invariance and balanced growth subsection. This parametric example is therefore special only insofar the various elasticities and logarithmic derivatives have been assumed to be constant.

We use the preference specification of (40), that is,

$u(c, n)=\frac{[\operatorname{cv}(n)]^{1-\eta}}{1-\eta}$

with $\eta \neq 1$. We restrict $v(n)$ to be strictly positive and such that $u(c, n)$ satisfies strict concavity and strict monotonicity. Define its logarithmic derivative,

$\ell_{v}(n)=\frac{v^{\prime}(n) n}{v(n)}$.

Note that $\ell_{v}(n)<0$. As before, we let

$\ell_{v, t}=\ell_{v}\left(\bar{n}_{t}\right)$,

where $\bar{n}_{t}$ is some chosen benchmark.

The utility function is

$U_{t, t}=\frac{\left(\left[c_{t, y} v\left(n_{t}\right)\right]^{1-\eta}+\beta\left\{E_{t}\left[\left(\varpi_{t+1}^{\eta} c_{t+1, o}^{1-\eta}\right)^{1-v / 1-\eta}\right]\right\}^{1-\eta / 1-\nu}\right)^{1-\xi / 1-\eta}}{1-\xi}$.

In terms of our general specification,

$$
\begin{aligned}
& q(c)=\frac{c^{1-\eta}}{1-\eta}, \\
& x(q)=\frac{[(1-\eta) q]^{1-\nu / 1-\eta}}{1-\nu}, \\
& z(a)=\frac{[(1-\eta) q]^{1-\xi / 1-\eta}}{1-\xi} .
\end{aligned}
$$

The argument share $\alpha_{t}$ is given per 


$$
\frac{1}{\alpha_{t}}=1+\beta \bar{\varpi}_{t+1}^{\eta}\left[\frac{\bar{c}_{t+1, o}}{\bar{c}_{t, y} v\left(\bar{n}_{t}\right)}\right]^{1-\eta} .
$$

Preferences and the Length of Life If $\eta<1$ and given a total amount $c_{t+1,0}$ of consumption when old, agents prefer a longer life with a fairly small amount of consumption per unit of time to a shorter life with a fairly large amount of consumption per unit of time. This is not so, however, if $\eta>1$ with the preference specification above, and indeed, the utility function then even has the feature, that old agents strictly prefer a shorter life to a longer life. Put differently, committing suicide is optimal. This is obviously an undesirable feature. As a further consequence, note that with the previously defined preferences, the limit for $\eta \rightarrow 1$ is not well defined.

There are at least three ways to resolve this conundrum. One possibility is to modify preferences when old so as to generate strictly positive utility, whenever the agent is alive. This can be done, for example, by modifying

$$
q(c)=\frac{(c+\underline{c})^{1-\eta}-\underline{c}^{1-\eta}}{1-\eta}
$$

for some baseline level of consumption $\underline{c}>0$. With this, total utility while old is

$$
\begin{aligned}
\varpi q\left(\frac{c}{\varpi}\right)= & \varpi\left[\frac{\left(\frac{c}{\varpi}+\underline{c}\right)^{1-\eta}-\underline{\underline{c}}^{1-\eta}}{1-\eta}\right] \\
& \rightarrow \varpi\left[\log \left(\frac{c}{\underline{c} \omega}+1\right)\right](\eta \rightarrow 1),
\end{aligned}
$$

which exists and is always guaranteed to be positive, but has a nonconstant elasticity in $\underline{\underline{c}}$ as consumption grows (holding $\underline{\underline{c}}$ constant).

The second possibility is to let agents compare utility of consumption $c$ while alive to some benchmark level of consumption $c$ (possibly dependent on the date $t$ ), while alive, and otherwise assign zero utility,

$q(c)=\frac{c^{1-\eta}-\underline{c}^{1-\eta}}{1-\eta}$

so that the total utility for old agents is given by 


$$
\begin{aligned}
\varpi q\left(\frac{c}{\varpi}\right)= & {\left[\frac{\left(\frac{c}{\varpi}\right)^{1-\eta}-\underline{c}^{1-\eta}}{1-\eta}\right] } \\
& \rightarrow \varpi\left[\log \left(\frac{c}{c \varpi}\right)\right](\eta \rightarrow 1),
\end{aligned}
$$

which exists and has a constant elasticity as $c$ grows, but may no longer be guaranteed to be always positive.

The third possibility is to restrict $\eta<1$ for the original preference specification. At the expense of generality but at the gain of some simplification for the analytics, we shall proceed with this restriction.

In either of these scenarios, the social planner effectively contemplates insurance against the utility loss (or gain!), when dead. Put differently, if there is a longevity shock, the old are happier simply due to living longer. Hence, the social planner may seek to redistribute the gains in utility of the old to the young.

These insurance motives, while present in this paper, merit deeper philosophical thought. Suppose, for example, some medical treatment could be found that extends the life of the old but is rather costly. How much should it be worth to society, and who should pay for it? This depends rather crucially on the additional utility generated due to being alive. For the third possibility, that is, our original specification and with $\eta<1$, the additional utility generated is bounded.

With $\eta>1$ and the specification (46), the utility gain is unbounded, as $\underline{c} \rightarrow 0$; that is, death can be made to be arbitrarily unattractive. In that case, the cost limit ultimately is the entire GDP. To see this, suppose that total average consumption of the old is given by $c=c(\varpi)$, reflecting the cost of improving longevity and the opportunity costs of transferring consumption from the young to the old. The first-order condition with respect to $\varpi$ is given by

$$
0=\frac{\left[\frac{c(\boldsymbol{\omega})}{\boldsymbol{\omega}}\right]^{1-\eta}-\underline{c}^{1-\eta}}{1-\eta}-\left[\frac{c(\boldsymbol{\omega})}{\boldsymbol{\omega}}\right]^{1-\eta}\left(1-\ell_{c}\right),
$$

where

$\ell_{c}=\frac{c^{\prime}(\varpi) \varpi}{c(\varpi)}$. 
For any value $c(\varpi) / \varpi>0$ and $\ell_{c}$, there is some $\underline{c}>0$, so that this derivative is positive; that is, unless one obtains direction observations on choices involving the length of life, there is always some specification for the "fear of death," parameterized by $\underline{c}$, which would justify additional spending on life-prolonging measures, no matter how costly.

Ultimately, from an economic perspective, this raises the need for measuring the aversion of agents against death in data, and using it to calibrate these preferences. There are a number of activities where agents clearly trade off the risk of dying against some utility-enhancing activity. For example, driving at a higher speed entails a higher risk of dying, and smoking causes people to die at an earlier age. By carefully measuring the utility gain (and measuring marginal changes, e.g., due to the change in the price of cigarettes or due to the higher safety of cars), one may be able to calculate an economic value for death. This provides appropriate limits for the amount of resources that should be spent on extending lives. The difficult discussions-when economic reasoning of this sort, combined with ethical and moral judgments, provide guidelines for the share of GDP to be spent on health care or, more drastically, turning off life-support measures for a terminally ill patient-have only begun, and will surely intensify in the future, as the technological possibilities advance (see e.g., Murphy and Topel [2002] or Hall and Jones [2004]).

\section{Optimal Risk Sharing}

PROPOSITION 3 [Risk-Sharing with Epstein-Zin preferences.] With the preferences given by (42) and (44) and up to a first-order approximation in the $\log$ deviations around a chosen benchmark, optimal intergenerational risk sharing implies

$$
(\xi-\eta) \check{a}_{t}^{*}+\eta \check{c}_{t, y}-(1-\eta) \ell_{v, t} \check{n}_{t}=(\xi-\eta) \check{a}_{t-1}^{*}+\check{d}_{t}+\eta\left(\check{c}_{t, o}-\check{\varpi}_{t}\right),
$$

where

$$
\begin{aligned}
& \check{a}_{t}^{*}=\alpha_{t}\left(\check{c}_{t, y}+\ell_{v, t} \check{n}_{t}\right)+\left(1-\alpha_{t}\right)\left[\frac{\eta}{1-\eta} E_{t}\left(\check{\varpi}_{t+1}\right)+E_{t}\left(\check{c}_{t+1, o}\right)\right] . \\
& \check{d}_{t}=(v-\eta)\left\{\frac{\eta}{1-\eta}\left[\check{\varpi}_{t}-E_{t-1}\left(\check{\varpi}_{t}\right)\right]+\left[\check{c}_{t, o}-E_{t-1}\left(\check{c}_{t, o}\right)\right]\right\}
\end{aligned}
$$

where 
$\check{a}_{t}^{*}=\left(\frac{\check{a}_{t}}{1-\eta}\right)$.

PROOF. Note first, that for any function $f(x)=x^{\alpha}$, we have $\ell_{f}=\alpha$ and $\mu_{f}=$ $1-\alpha$. Leaving away the time subscript $t$ except for $\mu_{u c, n, t}, \ell_{u, n, t}$ and $\ell_{v, t}$, since everything else is now constant, calculate

$$
\begin{aligned}
\mu_{z} & =\frac{\xi-\eta}{1-\eta^{\prime}} \\
\mu_{u c, c} & =\eta, \\
\mu_{u c, n, t} & =-(1-\eta) \ell_{v, t^{\prime}} \\
\mu_{x} & =\frac{\nu-\eta}{1-\eta} \\
\mu_{q} & =\eta
\end{aligned}
$$

and

$$
\begin{aligned}
\ell_{u, c} & =1-\eta, \\
\ell_{u, n, t} & =(1-\eta) \ell_{v, t} \\
\ell_{x} & =\frac{1-v}{1-\eta}, \\
\ell_{q} & =1-\eta .
\end{aligned}
$$

Substitute into equation (31).

Note that we assume $\eta<1$, as discussed in the subsection on preferences and the length of life. Thus, leisure and first-period consumption are complements and $\mu_{u, m, t}<0$.

To provide some intution for the risk-sharing condition, it may be useful to consider the following six special cases.

1. Assume: no Epstein-Zin, constant labor. Result: perfect correlation of per-period consumption,

$\check{c}_{t, y}=\check{c}_{t, 0}-\check{\varpi}_{t}$.

2. Assume: additionally, endogenous labor. Result: since $\eta<1$, that is, since leisure and consumption of the young are complements, leisure moves in the same direction as consumption of the young relative to perperiod consumption of the old. To see this, rewrite 
$\check{c}_{t, y}-\frac{1-\eta}{\eta} \ell_{v, t} \check{n}_{t}=\check{c}_{t, o}-\check{\varpi}_{t}$

as

$\frac{1-\eta}{\eta}\left[(-1) * \ell_{v, t}\right]\left[(-1) * \check{n}_{t}\right]=\check{c}_{t, y}-\left(\check{c}_{t, o}-\check{\varpi}_{t}\right)$.

The intuition was discussed subsequent to proposition 2 .

3. Assume: the old are risk averse, $v>\eta$, while there is no additional life risk aversion, $\xi=\eta>0$. Benchmark uses information up to $t-1$.

Result: the old bear less of the risk. Longevity enters separately from old-age consumption.

$\check{c}_{t, y}-\frac{1-\eta}{\eta} \ell_{v, t} \check{n}_{t}=\frac{\frac{v}{\eta}-1}{1-\eta} \check{\varpi}_{t}+\frac{\nu}{\eta}\left(\check{c}_{t, o}-\check{\varpi}_{t}\right)$.

4. Assume: the old are risk averse, $\nu>0$. The young are risk neutral, $\xi=$ $v=0$. Assume constant labor. Result: the young bear all the risk,

$0=\check{c}_{t, o}-E_{t-1}\left(\check{c}_{t, o}\right)$.

5. Assume: infinite elasticity of intertemporal substitution, $\eta=0$, constant labor, equal risk aversion of young and old, $\nu=\xi>0$. Evaluate risk sharing based on information up to $t-1$. Result: the lifetime consumption of the young reacts as much as consumption of the old,

$\alpha_{t} \check{c}_{t, y}+\left(1-\alpha_{t}\right) E_{t}\left(\check{c}_{t+1, o}\right)=\check{c}_{t, o}-E_{t-1}\left(\check{c}_{t, o}\right)$.

6. Assume: infinite elasticity of intertemporal substitution, no risk aversion of the old $\eta=v=0$, constant labor, but risk aversion of young, $\xi>$ 0 . Evaluate the risk-sharing condition based on information up to and including $t-1$. Result: The old bear all the risk,

$0=\alpha_{t} \check{c}_{t, y}+\left(1-\alpha_{t}\right) E_{t}\left(\check{c}_{t+1, o}\right)$.

The parameter $\xi$ is most sensibly interpreted as life-risk aversion. Suppose $\check{c}_{t, o}=E_{t-1}\left(\check{c}_{t, 0}\right)$, that is, old-age consumption changes are predictable when these agents are young, and suppose that $\check{\varpi}_{t} \equiv 0$. Then, the right-hand side of equation (49) can be written as

$(\xi-\eta) \alpha_{t-1}\left(\check{c}_{t-1, y}+\ell_{v, t} \check{n}_{t-1}\right)+\left[(\xi-\eta)\left(1-\alpha_{t-1}\right)+\eta\right) \check{c}_{t, 0}$. 
The relevant scaling parameter on the rate of change for consumption of the old, $\check{c}_{t, o}$ is given by a combination of the curvature parameter $\eta$ of the utility of the young and life-risk aversion $\xi$. The curvature parameter of the young comes in because, with a foreseeable shock, the intertemporal elasticity of substitution between the two periods of life rather than the post-birth old-age risk aversion $\nu$ matters.

If relative consumption changes are the same, $\check{c}_{s, 0}=\check{c}_{s-1, y}$, for $s=t$ and $s=t-1$, and if labor stays constant, $\check{n}_{t}=\check{n}_{t-1}=0$. then $\check{a}_{s}^{*}=\check{c}_{s, y}=\check{c}_{s+1,0}$ for $s=t$ and $s=t-1$, and the risk-sharing condition (49) for a foreseeable shock becomes

$\check{c}_{t, y}=\check{c}_{t, o}$

as in (34). That is, young and old should share consumption risk equally (in proportion to their benchmark level), when faced with a foreseeable shock. Their relevant risk aversion is now the life-risk aversion $\xi$, which is the same for both generations.

With unanticipated changes, by contrast-that is, if $E_{t-1}\left(\check{c}_{t, 0}\right)=\check{c}_{t, y}=0$, and assuming fixed employment and longevity, as well as $\check{c}_{t, y}=E_{t}\left(\check{c}_{t+1,0}\right)$, the risk-sharing condition (49) becomes

$\xi \check{c}_{t, y}=\nu \check{c}_{t, o}$.

Whereas old-age risk aversion $v$ is relevant for consumption of the old in this case, life-risk aversion $\xi$ applies to the young. Indeed, in contrast to the young, who can change all the arguments of their utility function, the old can only adjust their old-age consumption in response to an unanticipated shock. Equation (53) is a version of one of the results announced in the introduction: the old bear a proportionally larger share of the consumption risk, if their risk aversion is lower than the corresponding life-risk aversion. In section 5.6 we shall see that this result continues to hold approximately for reasonable parameters, when preferences toward leisure are introduced.

\subsection{Solving the Social Planner's Problem and Parametric Choices}

Assume from now on that preferences are given by equation (44).

To turn the endogenous variables into a stationary system, define $\tilde{c}_{t, y}=\frac{c_{t, y}}{k_{t-1}}$ 


$$
\begin{gathered}
\tilde{c}_{t, o} \equiv \frac{c_{t, 0}}{k_{t-1}}, \\
\tilde{w}_{t} \equiv \frac{w_{t}}{k_{t-1}}, \\
\tilde{y}_{t} \equiv \frac{y_{t}}{k_{t-1}}, \\
\tilde{k}_{t} \equiv \frac{k_{t}}{k_{t-1}}, \\
\tilde{x}_{t} \equiv \frac{x_{t}}{k_{t-1}},
\end{gathered}
$$

except that we do not detrend $n_{f}$ and that we define

$$
\tilde{a}_{t} \equiv \frac{a_{t}}{k_{t-1}^{\mathrm{I}-\eta}}
$$

$\tilde{s}_{t} \equiv s_{t} k_{t}^{v-\xi}$,

where

$s_{t} \equiv\left[(1-\eta) a_{t}\right]^{\xi-\eta / 1-\eta} x_{t}^{\eta-v}$

The exponent of the normalization variable differs here in order to achieve the appropriate scaling for these utility variables. Moreover, note that we scale $s_{t}$ by $k_{t}^{\nu-\xi}$ rather than by $k_{t-1}^{\nu-\xi}$ because that way, $\tilde{s}_{t-1}$ (rather than $\tilde{s}_{t-1}$ and $\tilde{k}_{t-1}$ ) turns out to be the only remaining endogenousstate variable.

One can then solve for a steady state in these detrended variables as well as calculate the dynamics around this steady state using log linearization. The details are available in the appendix. Here we highlight only some key results.

\subsubsection{Parametric Choices}

To explicitely calculate the steady state and provide quantitative results, we introduce a number of parametric assumptions regarding preferences, technologies, and shocks.

For preferences regarding leisure, we assume, specifically, that 
$v(n)=\bar{v} \frac{(1-n)^{1-\chi}}{1-\chi}$

with $0 \leq \chi<1$. Consequently,

$\ell_{v}(n)=-(1-\chi) \frac{n}{1-n}$

$\mu_{v}(n)=-\chi \frac{n}{1-n}$

For the production function $f(x)$, we assume

$f(x)=\left[\theta+(1-\theta) x^{1-(1 / \psi)}\right]^{1 /[1-(1 / \psi)]}$,

with $0 \leq \theta<1$ and $\psi>0$ (where one should note that we usually use $x$ $=Z n$ as argument), and thus

$\ell_{f}(x)=\frac{(1-\theta) x^{1-(1 / \Downarrow)}}{\theta+(1-\theta) x^{1-(1 / \psi)}}$,

$\mu_{f}(x)=\frac{1}{\psi}\left[1-\ell_{f}(x)\right]$.

For $\psi \rightarrow 1$, this becomes ${ }^{2}$

$f(x)=x^{1-\theta}$

with

$\ell_{f}(x)=1-\theta$,

$\mu_{f}(x)=\theta$.

For the stochastic part, let

$\zeta_{t}=\left[\begin{array}{c}\boldsymbol{\sigma}_{t} \\ A_{t} \\ Z_{t} \\ \pi_{t} \\ \delta_{t}\end{array}\right]$

be the vector of exogenous parameters. We assume a steady state $\bar{\zeta}$ exists and that 
$\hat{\zeta}_{t}=\log \left(\zeta_{t}\right)-\log (\bar{\zeta})$

follows an AR(1) process,

$\hat{\zeta}_{t}=N \hat{\zeta}_{t-1}+\varepsilon_{t}$

for some $5 \times 5$ matrix $N$ with nonexplosive roots, where

$\varepsilon_{t}=\left[\begin{array}{l}\varepsilon_{\sigma, t} \\ \varepsilon_{A, t} \\ \varepsilon_{z, t} \\ \varepsilon_{\pi, t} \\ \varepsilon_{\delta, t}\end{array}\right]$

is the vector of innovations for each exogenous parameter, with $\varepsilon_{\xi} \sim \mathcal{N}(0, \Sigma)$.

\subsubsection{The Steady State}

For general production functions and preferences, the steady-state equations yield the following relationships between the detrended variables,

$$
\begin{aligned}
& \frac{\bar{\pi} \bar{c}_{y}}{\bar{y}}=\frac{\ell_{f}(\bar{Z} \bar{n})}{-\ell_{v}(\bar{n})}, \\
& \frac{\bar{c}_{o}}{\bar{\pi} \bar{c}_{y}}=\kappa_{3} \bar{k}^{(\xi-\eta) / \eta} v(\bar{n})^{(\eta-1) / \eta}, \\
& \bar{k}^{\xi}=\kappa_{4} \bar{R}=\kappa_{4}[\bar{A} f(\bar{Z} \bar{n})+1-\bar{\delta}],
\end{aligned}
$$

where

$\kappa_{3}=\frac{1}{\bar{\pi}}\left(\frac{\beta}{\omega}\right)^{1 / \eta} \overline{\bar{\sigma}} \exp \left[\frac{v-\eta}{\eta(1-v)} \frac{\sigma_{x}^{2}}{2}\right]$,

and

$\kappa_{4}=\omega \exp \left(\frac{\sigma_{R-v c}^{2}}{2}\right)$

where $\sigma_{R-v c}^{2}$ denotes the conditional variance of $\log R_{t+1}+\varsigma \log \left(\varpi_{t+1}\right)-$ $v \log \left(c_{t+1, o}\right)$ and $\sigma_{x}^{2}$ is the conditional variance of $\varsigma \log \varpi_{t+1}+(1-v) \log$ $c_{t+1, o}$ where $\varsigma=[\eta /(1-\eta)](1-v)$. These equations provide insight into the 
fraction of output spent on consumption of the young and the old, as well as the endogenous growth factor $\bar{k}$.

Substituting these equations into the equation on feasibility

$$
\frac{\bar{\pi} \bar{c}_{y}}{\bar{y}}\left(1+\frac{\bar{c}_{o}}{\bar{\pi} \bar{c}_{y}}\right)=1+\frac{1-\bar{\delta}-\bar{\pi} \bar{k}}{\bar{A} f(\bar{Z} \bar{n})}
$$

leads to a single nonlinear equation in $\bar{n}$,

$$
\begin{aligned}
& {\left[\frac{\ell_{f}(\bar{Z} \bar{n})}{-\ell_{v}(n)}\right]\left(1+\kappa_{3}\left\{\kappa_{4}[\bar{A} f(\bar{Z} \bar{n})+1-\bar{\delta}]\right\}^{\xi-\eta / \delta \eta} v(\bar{n})^{\eta-1 / \eta}\right)} \\
& =1+\frac{1-\bar{\delta}-\bar{\pi}\left\{\kappa_{4}[\bar{A} f(\bar{Z} \bar{n})+1-\bar{\delta}]\right\}^{1 / \xi}}{\bar{A} f(\bar{Z} \bar{n})} .
\end{aligned}
$$

With the parametric choice for preferences (i.e., $v(n)=\bar{v}\left[(1-n)^{1-x}\right] /$ $(1-\chi)$ ) and the production function (i.e., $\left.f(x)=\left[\theta+(1-\theta) x^{1-(1 / 4)}\right]^{1 /(1-(1 / \psi) 1}\right)$, the Appendix analyzes a special case in which the steady-state solution for employment can be calculated in closed form.

\subsubsection{The Dynamics}

After detrending with $k_{t-1}$, the model no longer contains a state variable, except

$\tilde{s}_{t}=s_{t} k_{t}^{v-\xi}$.

This variable arises solely from the intertemporal optimization of the social planner, and disappears if, for example, $\eta=\xi$, that is, if lifetime risk aversion equals the inverse of the intertemporal elasticity of substitution. One can think of $\tilde{s}_{t}$ as representing a utility promise to future generations, or as a device that allows the social planner to share a risk across many generations, if there is additional lifetime risk aversion.

Using log linearization and assuming a recursive law of motion in which $\hat{s}_{t-1}$ is the only-state variable, one can show that the dynamics can be reduced to solving a third-degree polynomial.

$0=\theta_{s}+\theta_{s, s} \varphi_{s, s}+\theta_{s, s} \varphi_{s, s}^{2}+\theta_{s, s s} \varphi_{s, s}^{3}$

for some coefficients $\theta_{s}, \theta_{s, s}, \theta_{s, s}$, and $\theta_{s, s s s}$. The explicit calculations can be found in Appendix subsection 5.A.5.

Equation (63) generally has three roots, $\varphi_{s, s, i}, i=1,2,3$, and for which closed-form solutions are available. If only one of these roots is stable, that is, less than 1 in absolute value, then this is the root we use. If more than one stable root exists, then an additional state variable is needed, as 
generally would be necessary to solve the system. If there are no stable roots, the system is explosive and our baseline assumption that there is a stationary solution to the social planner's problem in the detrended variables is unjustified.

When computing the following results, we use the toolkit implementation (see Uhlig [1999]), and allow for up to five rather than one endogenous-state variable, mostly for accounting reasons (see the Appendix).

\subsection{Decentralization and Generational Accounting}

The social planner's solution can be implemented in a decentralized economy using lump-sum taxes and transfers on young and old agents and possibly a return subsidy to old agents, when privately holding capital in order to equate the social and the private rate of return.

We assume that production occurs by a competitive sector of firms, renting capital and hiring labor from competitive households and experiencing an externality in production. More precisely, assume that production by firm $j$ is given by

$$
Y_{t, j}=A_{t} K_{t-1, j} f\left[Z_{t}\left(\frac{K_{t-1}}{\Pi_{t}}\right) \frac{N_{t, j}}{K_{t-1, j}}\right]
$$

where $N_{t, j}$ is the amount of labor hired by firm $j$ and $K_{t-1, j}$ is the amount of capital rented by firm $j$. The term $\left(K_{t-1} / \Pi_{t}\right)$ is an externality, enhancing labor productivity in proportion to the capital available per young person. Assuming that firms hire workers at their marginal product and rent capital at its marginal product on competitive markets, one can easily show that the capital-labor ratio across all firms is the same, and that the aggregate production function becomes (7).

The private rate of return to investing in capital is given by

$$
R_{t}^{\text {priv }}=R_{t}^{\text {soc }}-A_{t} f^{\prime}\left(Z_{t} n_{t}\right) Z_{t} n_{t}
$$

where we recall the social rate of return from equation (24) as

$$
R_{t}^{\text {soc }}-A_{t} f\left(Z_{t} n_{t}\right)+1-\delta_{t} \text {. }
$$

Thus, the private rate of return is diminished by the externality in accumulating capital, which is measured by the total wage payments per unit of capital,

$A_{t} f^{\prime}\left(Z_{t} n_{t}\right) Z_{t} n_{t}=\frac{w_{t} \Pi_{t} n_{t}}{K_{t-1}}=\frac{w_{t} \pi_{t} n_{t}}{k_{t-1}}$ 
Note that wages are given by (21), that is,

$w_{t}=A_{t} k_{t-1} f^{\prime}\left(Z_{t} n_{t}\right) \frac{Z_{t}}{\pi_{t}}$.

We assume that the budget constraint of the individual household is given by

$c_{t, y}+s_{t}+\tau_{t, y}=w_{t} n_{t}$

$c_{t+1,0}+\tau_{t+1,0}=\left(1+\sigma_{t+1}\right) R_{t+1}^{\text {priv }} s_{\sharp}$

where $s_{t}$ is private savings date $t, \tau_{t, y}$ is a lump-sum tax when young, $\tau_{t+1, o}$ is a lump-sum tax when old, and $\sigma_{t+1}$ is a return subsidy.

We shall focus on two extreme scenarios. In the first-call it the case of "private capital" - all capital is held privately and the government budget balances period by period,

$s_{\mathrm{t}}=k_{\mathrm{t}}$.

Define the tax share as

$\tau_{t, \text { priv }}=\frac{\tau_{t, y}}{w_{t} n_{t}}=1-\frac{c_{t, y}}{w_{t} n_{t}}-\frac{k_{t}}{w_{t} n_{t}}$

in order to express the magnitude of the lump-sum taxes more intuitively in proportion to the wage earnings of the young. One may want to view the average value of this share as reflecting the desire of the social planner toward redistribution, while its fluctuations may be viewed as fluctuating insurance payments of the young to the old in response to realizations of macroeconomic risks.

In this scenario, it is necessary to subsidize the returns to capital in proportion to capital held by the individual agent so that the total proportional subsidy amounts to giving the entire production, including the capital stock net of depreciation to the capital-holding old agents, and in turn lump-sum taxing the old agents so that the government budget constraint balances,

$$
\begin{aligned}
\sigma_{t+1, o} & =\frac{R_{t+1}^{\text {sox }}}{R_{t+1}^{\text {priv }}}-1, \\
& =\frac{w_{t+1} \pi_{t+1} n_{t+1}}{R_{f+1}^{\text {priv }} k_{t}}, \\
\tau_{t+1, o} & =\sigma_{t+1, o} R_{t+1}^{\text {priv }} k_{t}-\pi_{t+1} \tau_{t+1, y} \\
& =\pi_{t+1}\left(c_{t+1, y}+k_{t+1}\right) .
\end{aligned}
$$


One interpretation of this last equation is that the generational account balances-that is, the generational account of the old equals minus the generational account of the young.

In the second scenario-call it "the case of public capital"-all capital is held by pension funds,

$s_{t}=0$.

Thus, the lump-sum taxes to be paid by the young are payments to pension funds, which, in turn, finance old-age consumption. Note that this is a mixture of a pay-as-you-go system and a fully funded system. In this case, the payments by the young to the pension system are

$\tau_{t, \mathrm{pub}}=\frac{\tau_{t, y}}{w_{t} n_{t}}=1-\frac{c_{t, y}}{w_{t} n_{t}}$.

We examine both.

A defined benefit system can be viewed as a system where the old do not bear any of the risk (except perhaps longevity), whereas the old bear the entire risk of random returns in a defined contribution system. This analysis considers the degree of optimal risk sharing between young and old. The relationship between the solution to the social planner's problem, as investigated here, and these more-specific, "real world" pension systems, will be investigated in future research.

\subsection{Quantitative Results}

This section explores the quantitative properties of the model and numerically calculates the reaction of the various quantities to the relevant shocks. We use a hat on variables to denote the logarithmic deviation from the expected balanced growth path with $\tilde{s}_{t}$ constant (see Appendix subsection Loglinearization for details).

\subsubsection{Benchmark Parameterization}

As a benchmark parameterization, pick $\omega=\beta=0.4, \psi=1, \eta=0.5, \bar{\delta}=$ 1 , and $\xi=v=2$. Set $\chi=.5, \theta=1 / 3, \overline{\boldsymbol{\sigma}}=1, \bar{\pi}=1, \bar{Z}=1$, and let $\bar{Z}$ such that there is no growth in steady state, $\bar{k}=1$, requiring $\bar{A}=2.825$.

The resulting benchmark equilibrium has $\bar{n}=0.831, \overline{c_{y}} / \bar{y}=0.27, \bar{c}_{o} / \bar{c}_{y}$ $=1.22$, and $\bar{R}=2.5$, which corresponds to an annualized interest rate of 3.1 percent, assuming that one period lasts 30 years.

The lump-sum taxes relative to the wage bill of the young is $\tau_{\text {priv }}=$ 
-0.65 percent; that is, in the case of privately held capital, the young agent would receive a fairly neglible subsidy (financed out of a lumpsum tax on the "rich old," who also finance their own return subsidy). The lump-sum payment to the pension fund, in case of publicly held capital, is $\tau_{\text {pub }}=59$ percent; that is, the young would pay somewhat more than half of their wage earnings into the fund.

We have assumed the exogenous parameters to be i.i.d., except for longevity $\varpi_{t}$, which we assume to be a random walk. For the latter, it seems plausible that medical progress is permanent. For the other variables, note that, for example, total factor productivity (TFP) has a unit root due to the endogenous growth feature of our model, even though the TFP parameter $A_{t}$ (and the labor-productivity parameter $Z_{t}$ ) is i.i.d. Also, note that $\pi_{t}$ denotes population growth, so that the log of population follows a random walk if $\pi_{t}$ is i.i.d.

\subsubsection{Comparative Statics}

The sensitivity of these results to three parameters, $\xi, \delta$, and $\omega=\beta$ can be seen in Table 5.3. The last row in that table lists the feedback coefficient on the endogenous state $\hat{s}_{t}$ for the dynamic solution. While the first three columns have risk aversion of young and old the same, the last three columns set the life-risk aversion to the intertemporal elasticity of substitution of the young - that is, they do not modify the ex post utility of the young by a further risk transformation so that the socialintertemporal substitution elasticity coincides with the corresponding private elasticity. Note that the last three columns are fairly similar to the

Table 5.3

A comparison of steady states, when varying some parameters.

\begin{tabular}{|c|c|c|c|c|c|c|}
\hline$\xi=$ & 2.00 & 2.00 & 2.00 & 0.50 & 0.50 & 0.50 \\
\hline $\bar{\delta}=$ & 1.00 & 0.00 & 1.00 & 1.00 & 0.00 & 1.00 \\
\hline$\omega=\beta=$ & 0.40 & 0.40 & 0.80 & 0.40 & 0.40 & 0.80 \\
\hline $\bar{n}=$ & 0.83 & 0.80 & 0.83 & 0.83 & 0.83 & 0.83 \\
\hline $\bar{k}=$ & 1.00 & 1.17 & 1.00 & 1.00 & 1.94 & 1.00 \\
\hline $\bar{\pi} \bar{c}_{y} / \bar{y}=$ & 0.27 & 0.33 & 0.27 & 0.27 & 0.28 & 0.27 \\
\hline $\bar{c}_{o} / \bar{c}_{y}=$ & 1.22 & 1.81 & 1.22 & 1.22 & 1.20 & 1.22 \\
\hline$\tau_{\text {priv }}=$ & -0.65 & -21.83 & -0.65 & -0.56 & -59.68 & -0.56 \\
\hline$\tau_{\text {pub }}=$ & 59.38 & 50.35 & 59.38 & 59.37 & 57.62 & 59.37 \\
\hline$\varphi_{s, s}=$ & 0.15 & 0.19 & 0.12 & -0.00 & -0.00 & -0.00 \\
\hline
\end{tabular}


first three columns, except for this feedback coefficient and a substantial change for $\tau_{\text {priv }}$ in case of no depreciation.

Table 5.3 shows which factors determine optimal intergenerational distribution. In particular, the sign of the variable $\bar{\tau}_{\text {priv }}$ indicates to what extent the optimal pension system is funded. A positive value for $\bar{\tau}_{\text {priv }}$ indicates that the pension system is in part pay-as-you-go financed. In that case, the generational account for the young is negative (and for the old is positive) in the absence of shocks. There is systematic redistribution from the young to the old.

In the benchmark calculation, systematic redistribution is limited. The benchmark social optimum thus calls for a small amount of systematic redistribution from the old to the young in the decentralized economy.

The second column of table 5.3 shows that the systematic redistribution toward the young is increased if the current old become richer compared to the young on account of a lower depreciation rate (more capital income compared to labor income). Note also that the growth rate $\bar{k}$ increases. The higher return on capital (as a result of a lower depreciation rate) allows for a higher growth rate. The young save more because of two reasons: a higher return (substitution effect) and higher income (since the old transfer more income to the young). Another way of interpreting the increased systematic redistribution from the old to the young (an inverse pay-as-you-go [PAYG] system) is that the pension system is overfunded. Intuitively, funding increases because the return on capital increases and the older generation becomes richer.

A comparison of the second and fifth column of table 5.3 shows that the additional systematic redistribution in favor of the young due to a lower depreciation rate becomes more substantial if ex ante risk aversion $\xi$ decreases. The reason is that there is a lower social preference for similar utility levels across generations. Hence, making future generations better off compared to the current generation (as a result of higher return on capital and thus growth) becomes more attractive, and systematic redistribution from the old to the young is further increased. The old keep less of their additional income, as a result of higher capital income due to a lower depreciation rate.

Obviously, this systematic redistribution between young and old would be heavily affected by letting the discount factor of the social planner $\omega$ and the discount factor of the private agent $\beta$ differ. As an alternative possibility for comparing steady states, one could calibrate $\omega$ and $\beta$ in such a way that laisse $z$ faire in intergenerational distribution is 
optimal in the absence of shocks-that is, $\tau_{\text {priv }}=0$. Then, the decentralized economy with private capital yields the correct intergenerational distribution, assuming that the old pay their own investment subsidy.

\subsubsection{Endogenous Dynamics}

In table 5.3, and focusing on the last row in the last three columns, note that there is no feedback on the endogenous state (i.e., $\varphi_{s, s}$ ), if the lifetime risk aversion $\xi$ equals the intertemporal elasticity of substitution $\eta$; that is, if the social intertemporal substitution elasticity coincides with the private intertemporal substitution elasticity.

We are particularly interested in exploring the risk-sharing features as the risk aversion of the old, $v$, is varied vis-a-vis the life-risk aversion of the young, $\xi$. Figure 5.1 shows the dependence of the feedback coefficient $\varphi_{s, s}$ as these two risk-aversion parameters $v$ and $\xi$ are varied between $\eta=0.5$ and the value 4 at the upper end. As one can see, the en-

\section{Feedback coeff. on the state s}

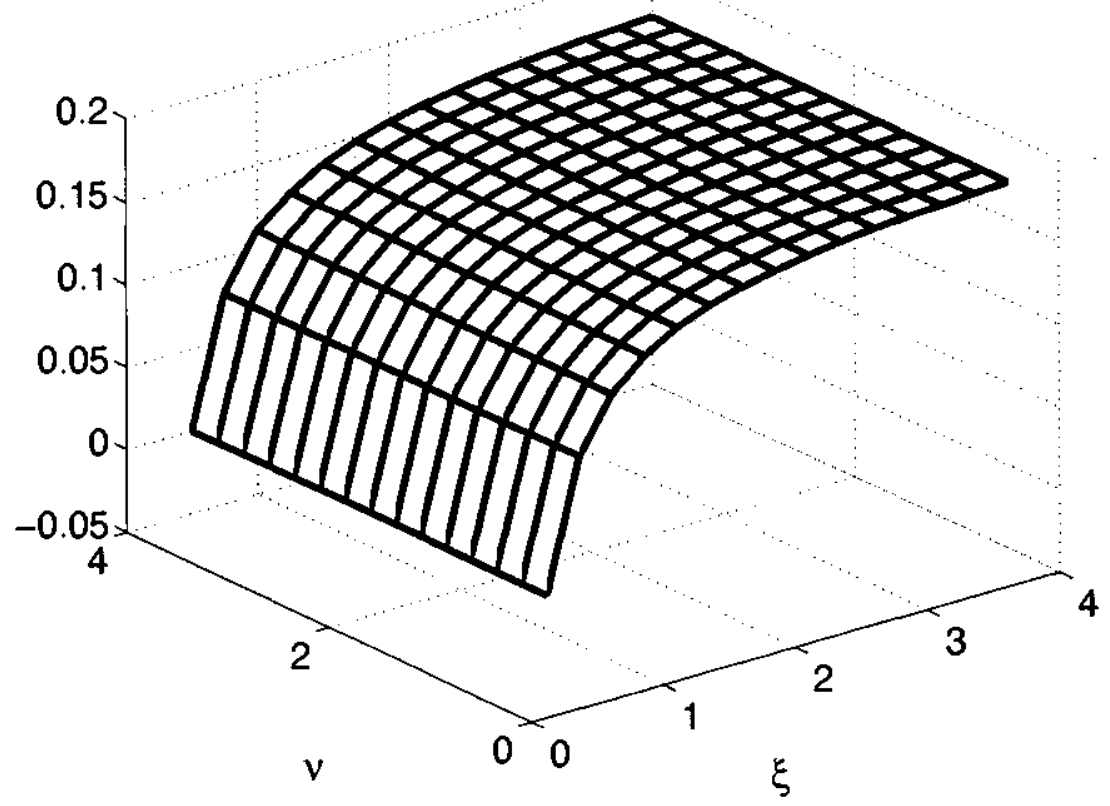

Figure 5.1.

Endogenous dynamics, that is, feedback coefficient $\varphi_{s s^{\prime}}$ on the endogenous state, as the risk-aversion parameters are varied. 
dogenous dynamics depend practically entirely on $\xi$ alone within this two-dimensional variation.

The impact of the state variable $\varphi_{s, s}$ is determined by the divergence between $\xi$ and $\eta$ and the importance of endogenous labor supply (i.e., the size of $\chi$; if $\chi=1$, labor supply is exogenous). The more difficult it becomes to substitute across generations compared to intertemporal substitution within generations as measured by $\eta$, the more shocks are spread out across various generations, as indicated by a larger feedback impact of the state variable.

Shifting risks between the old and the young when the shocks hit is sufficient if $\xi=\eta$. In that case, the young affect their saving behavior in the socially optimal way to redistribute between generations and the government does not have to perform any additional redistribution next period.

\subsubsection{Intergenerational Risk Sharing}

Table 5.4 contains the corresponding feedback coefficients to three shocks: total factor productivity $Z$, longevity $\varpi$, and population growth $\pi$. There, $\varphi_{\text {growth }}$, refers to the absolute change in the growth rate (rather than relative to the growth factor $\bar{k}$ ). If, for example, $\varphi_{\text {growth }, z}=1.28$, then this means that the capital stock will grow by an additional 1.28 percent, if TFP increases by 1 percent. Likewise, $\phi_{\tau, \text { priv },}=-0.35$ says that the lump-sum tax to be paid in case of privately held capital is lowered by 0.35 percent of the current wage bill, in case TFP increases by 1 percent.

To shed light on risk sharing, we have included three more quantities. First and second, we have calculated the reaction coefficient for the total tax collection from the young, normalized by the unchanged wage earnings, each for the case of private as well as public capital. In the case of private capital, this is

$$
\begin{aligned}
\tau_{\text {privtot }} & =\frac{\pi_{t} \tau_{t, y}}{\bar{\pi} \bar{w} \bar{n}} \\
& =\frac{\pi_{t}\left(w_{t} n_{t}-c_{t, y}-k_{t}\right)}{\bar{\pi} \bar{w} \bar{n}}
\end{aligned}
$$

and its percentage change is given by

$\hat{\tau}_{\text {privtot }}=\hat{\tau}_{\text {priv }}+\bar{\tau}_{\text {priv }}(\hat{n}+\hat{w}+\hat{\pi})$ 
Table 5.4.

Parameter variations and feedback coefficients.

\begin{tabular}{|c|c|c|c|c|c|c|}
\hline$\xi=$ & 2.00 & 2.00 & 2.00 & 0.50 & 0.50 & 0.50 \\
\hline $\bar{\delta}=$ & 1.00 & 0.00 & 1.00 & 1.00 & 0.00 & 1.00 \\
\hline$\omega=\beta=$ & 0.40 & 0.40 & 0.80 & 0.40 & 0.40 & 0.80 \\
\hline$\varphi_{c y} z$ & 1.00 & 0.71 & 1.00 & 1.38 & 0.83 & 1.38 \\
\hline$\varphi_{c o} z$ & 1.00 & 0.72 & 1.00 & 0.30 & 0.23 & 0.30 \\
\hline$\varphi_{12} z$ & 0.00 & 0.07 & -0.00 & -0.07 & 0.03 & -0.07 \\
\hline$\varphi_{y} z$ & 1.00 & 1.04 & 1.00 & 0.95 & 1.02 & 0.95 \\
\hline$\varphi_{\text {growth }} z$ & 1.00 & 0.91 & 1.00 & 1.20 & 1.77 & 1.20 \\
\hline$\varphi_{\text {t.priv. }} z$ & 0.00 & 0.36 & 0.00 & -0.32 & 0.21 & -0.32 \\
\hline$\tau_{\text {privtot }}$ for $Z$ & -0.01 & 0.13 & -0.01 & -0.33 & -0.40 & -0.33 \\
\hline$\varphi_{\text {T,pub }} z$ & 0.00 & 0.17 & -0.00 & -0.17 & 0.08 & -0.17 \\
\hline$\tau_{\text {pub,tot }}$ for $Z$ & 0.59 & 0.69 & 0.59 & 0.39 & 0.67 & 0.39 \\
\hline$c_{0} / c_{\text {tot }}$ for $Z$ & 0.00 & 0.00 & -0.00 & -0.49 & -0.27 & -0.49 \\
\hline$\varphi_{c y, \pi}$ & -0.69 & -0.58 & -0.67 & -0.87 & -0.90 & -0.87 \\
\hline$\varphi_{c 0, \pi}$ & -0.69 & -0.60 & -0.71 & -0.23 & -0.24 & -0.23 \\
\hline$\varphi_{n, \pi}$ & -0.06 & -0.10 & -0.06 & -0.02 & -0.02 & -0.02 \\
\hline$\varphi_{y, \pi}$ & -0.04 & -0.06 & -0.04 & -0.02 & -0.01 & -0.02 \\
\hline$\varphi_{\text {growth } \pi}$ & -0.74 & -0.80 & -0.75 & -0.93 & -1.84 & -0.93 \\
\hline$\varphi_{\tau, p r i v, \pi}$ & -0.32 & -0.52 & -0.33 & -0.11 & -0.12 & -0.11 \\
\hline$\tau_{\text {privtot }}$ for $\pi$ & -0.32 & -0.51 & -0.33 & -0.11 & -0.11 & -0.11 \\
\hline$\varphi_{\tau, \text { pub }, \pi}$ & -0.14 & -0.24 & -0.15 & -0.06 & -0.05 & -0.06 \\
\hline$\tau_{\text {pub,tot }}$ for $\pi$ & -0.17 & -0.27 & -0.18 & -0.07 & -0.05 & -0.07 \\
\hline$c_{v} / c_{\text {tot }}$ for $\pi$ & -0.45 & -0.36 & -0.47 & -0.16 & -0.15 & -0.16 \\
\hline$\varphi_{c y, m}$ & -0.93 & -0.85 & -1.02 & -3.49 & -7.95 & -3.49 \\
\hline$\varphi_{c 0, \pi}$ & -0.39 & -0.24 & -0.20 & -0.96 & -1.56 & -0.96 \\
\hline$\varphi_{n, w}$ & 0.18 & 0.19 & 0.19 & 0.66 & 1.57 & 0.66 \\
\hline$\varphi_{y, \pi}$ & 0.12 & 0.13 & 0.13 & 0.44 & 1.05 & 0.44 \\
\hline$\varphi_{\text {growth.w }}$ & 1.25 & 1.34 & 1.18 & 4.26 & 9.50 & 4.26 \\
\hline$\varphi_{\tau, p r i v, w}$ & -0.25 & -0.25 & -0.16 & -0.70 & -0.69 & -0.70 \\
\hline$\tau_{\text {privtot }}$ for $\pi$ & -0.25 & -0.28 & -0.17 & -0.70 & -1.31 & -0.70 \\
\hline$\varphi_{\text {T,pub,w }}$ & 0.43 & 0.48 & 0.47 & 1.60 & 3.81 & 1.60 \\
\hline$\tau_{\text {pub.tot }}$ for $\varpi$ & 0.50 & 0.55 & 0.54 & 1.86 & 4.42 & 1.86 \\
\hline$c_{0} / c_{\text {tot }}$ for $\varpi$ & 0.25 & 0.22 & 0.37 & 1.14 & 2.91 & 1.14 \\
\hline
\end{tabular}


Note that this coincides with the reaction coefficient for $\hat{\tau}_{\text {priv }}$ if $\bar{\tau}=0$. Likewise,

$\tau_{\text {pub tot }}=\frac{\pi_{t}\left(w_{t} n_{t}-c_{t, y}\right)}{\bar{\pi} \bar{w} \bar{n}}$

with

$\hat{\tau}_{\text {pub,tot }}=\hat{\tau}_{\text {pub }}+\bar{\tau}_{\text {pub }}(\hat{n}+\hat{w}+\hat{\pi})$

The total $\tau_{\text {privtot }}$ is the total contribution of the young to a pension system where capital is held privately. In particular, if it is identical to zero and unaffected by shocks, this means that the young do not contribute to insuring the old, and that the old have to bear the entire risk to the returns of their capital alone. Put differently, this number indicates additional intergenerational risk sharing compared to a decentralized equilibrium in which the old can save only through capital markets (and the old finance their own investment subsidy). The government's ability, in fact, to create new assets to allow trade of risks between generations is indicated by $\tau_{\text {priv }}$.

Third, we have also calculated the change in consumption of the old relative to the total change in consumption, that is, the feedback coefficient for the (log-linearized) quantity

rat $_{t}=\frac{c_{o, t}}{c_{t o t, t}}=\frac{c_{o, t}}{c_{o, t}+\pi_{t} c_{y, t}}$.

In log deviations,

$r a t_{t}=\frac{1}{1+\frac{\bar{c}_{o}}{\bar{\pi} \bar{c}_{y}}}\left(\hat{c}_{o, t}-\hat{c}_{y, t}-\hat{\pi}_{t}\right)$.

If this quantity is unchanged, that is, if the feedback is zero, then this means that consumption of the old increases in proportion to overall consumption resources. Generally, rât $>0$ in one of three cases. It happens if both consumptions rise, but $c_{t, 0}$ rises relatively more. It also happens if both consumptions fall, but $c_{t, o}$ falls relatively less. Finally, it happens if $c_{t, o}$ rises and $c_{t, y}$ falls. These three cases ought to be kept in mind when evaluating the results.

The dependence of the shock reaction of these three quantities, as well as labor supply on the two risk-aversion parameters $v$ and $\xi$, are shown in figures 5.2 through 5.5 , both as a three-dimensional mesh as well as a two-dimensional contour plot. 
Old oons. rel, to total, prod. shock

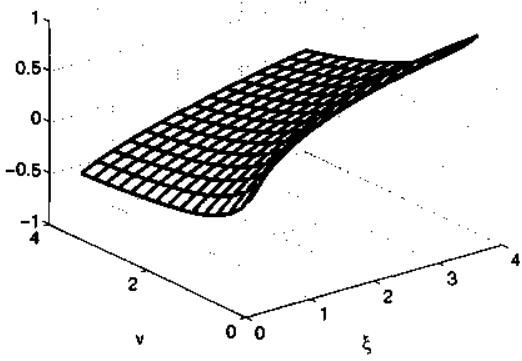

Ord cons. rel. to total, popul. shock

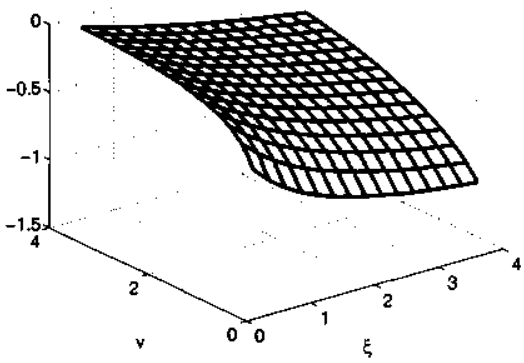

Old cons. rel. to total, longev. shock

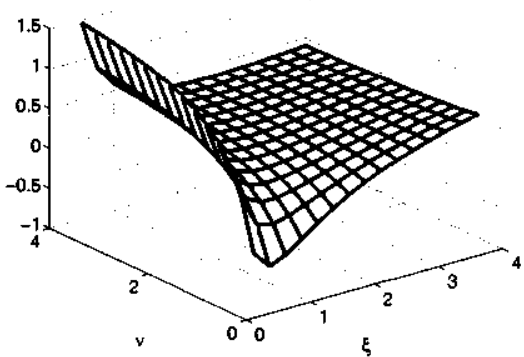

Labor, productivity shock

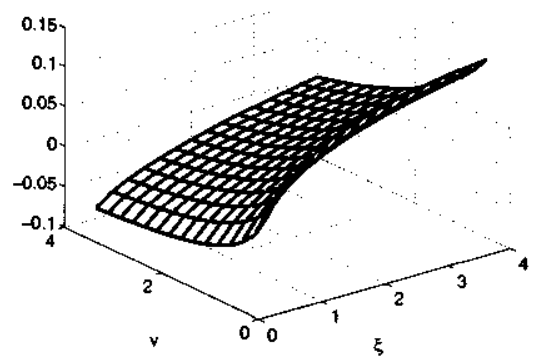

Labor, population shock

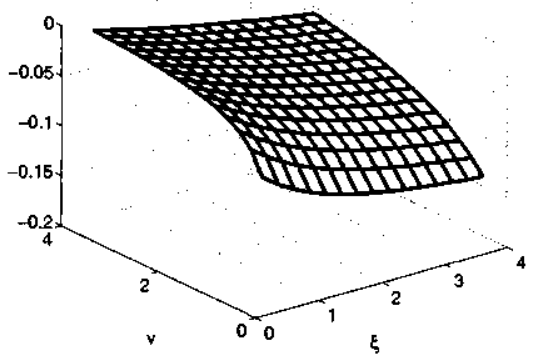

Labor, longevity shock

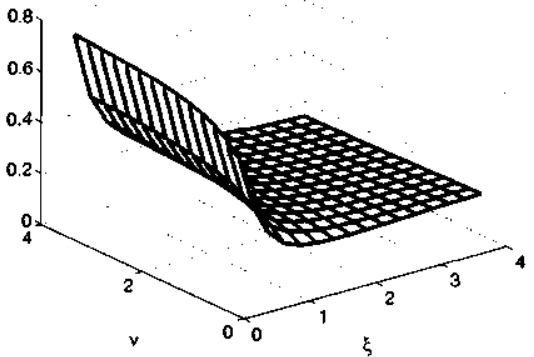

Figure 5.2.

Reaction of consumption of old relative to total consumption as well as reaction of labor, as the risk-aversion parameters are varied.

For the impulse responses, we have used the benchmark parameterization, except for setting $\xi=1$ rather than $\xi=2$. This does not change the steady state, but makes for a differential reaction of consumption of the young vis-a-vis consumption of the old (see the following discussion), and therefore for more differentiated impulse response figures. They are shown in figures 5.6 to 5.11 . The upper left corner shows the 

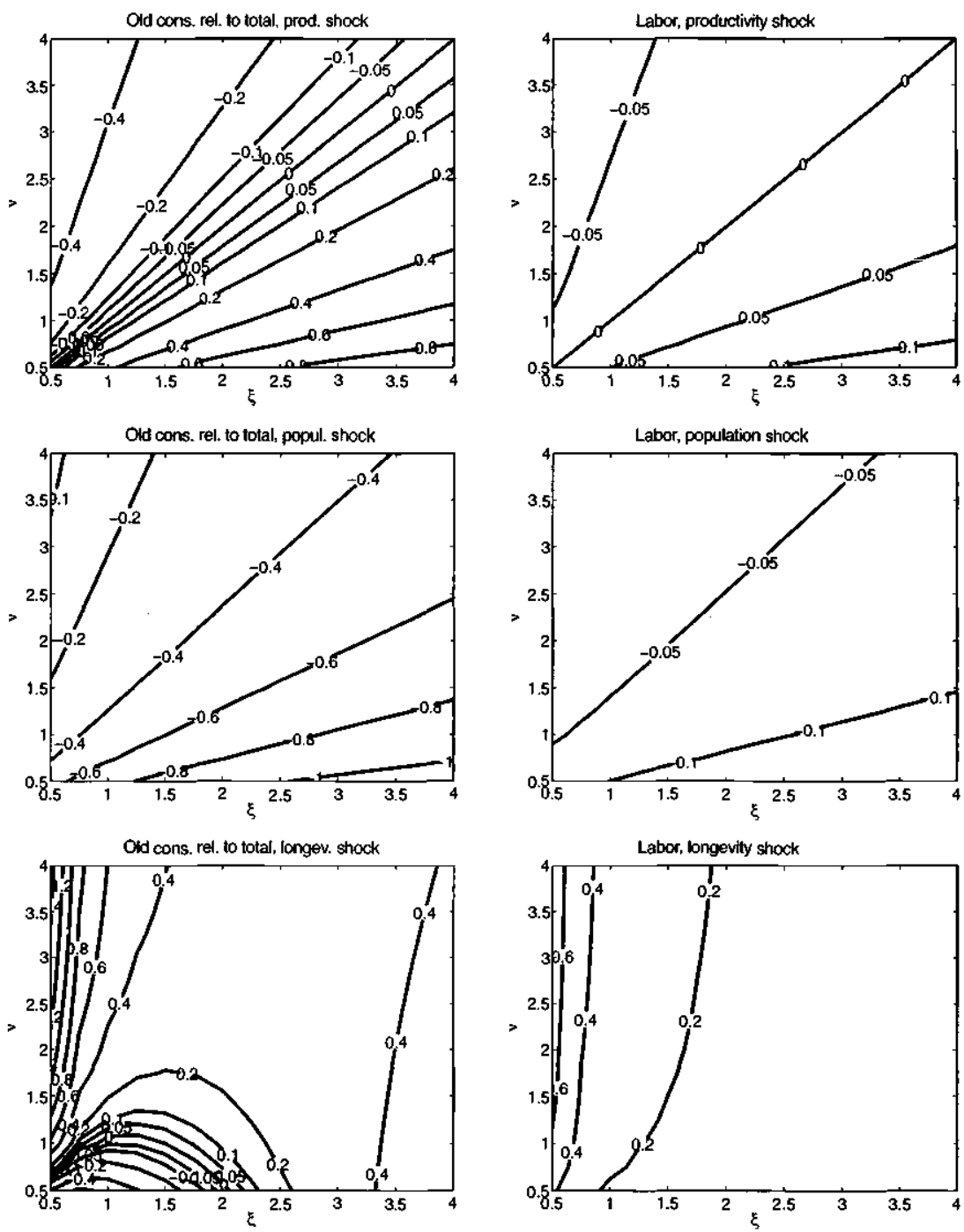

Figure 5.3.

Contour plots. Reaction of consumption of old relative to total consumption as well as reaction of labor, as the risk-aversion parameters are varied.

response of normalized consumption of the young, old, and the capital stock, whereas the lower left corner provides the corresponding level variables, that is, without dividing by aggregate capital. The upper right corner shows the responses of labor, output (normalized), and growth, while the lower right corner shows the responses of the lump- 
Tax ol young, priv. cap., prod. shock

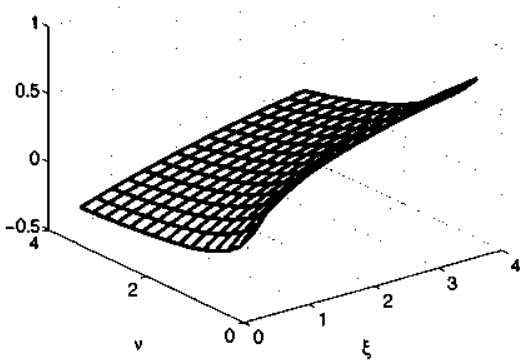

Tax of young, priv. cap., popul. shock

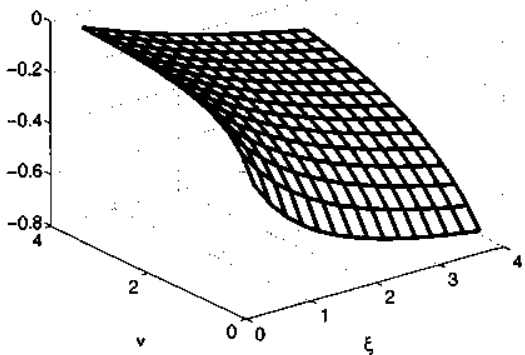

Tax of young, priv. cap., longev. shock

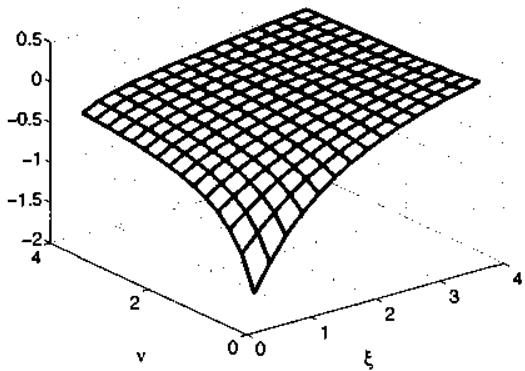

Tax of young, pub. cap., prod. shock

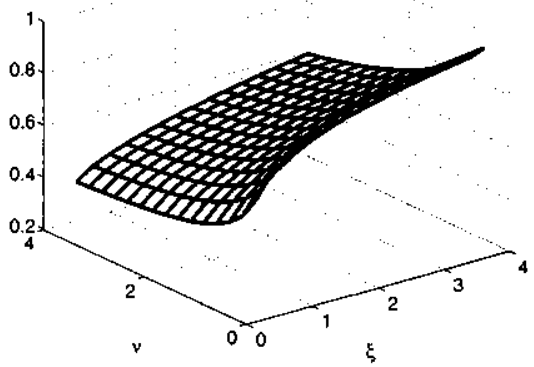

Tax of young, pub, cap., popul. shock

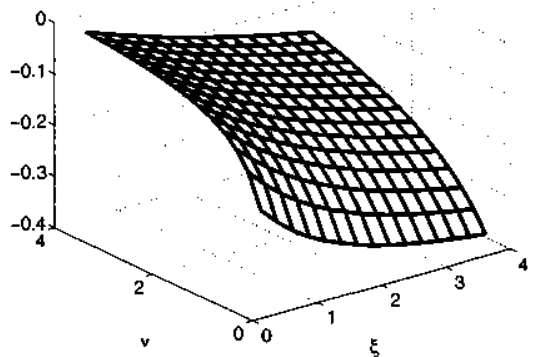

Tax of young. pub. cap. kngev. shock

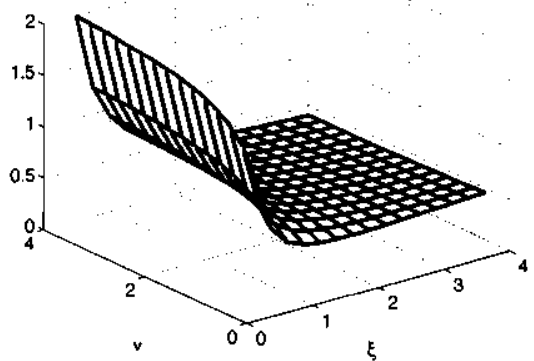

Figure 5.4.

Reaction of total tax payments to young relative to unchanged labor income, as the riskaversion parameters are varied.

sum taxes to be paid by the young, expressed as a percentage of the steady-state wage bill. Likewise, the response of depreciation is expressed as a percentage of the capital stock, not as a percentage of the steady-state depreciation rate (which is assumed to be zero, anyway). All figures also show the response of the shocked variable, that is, 

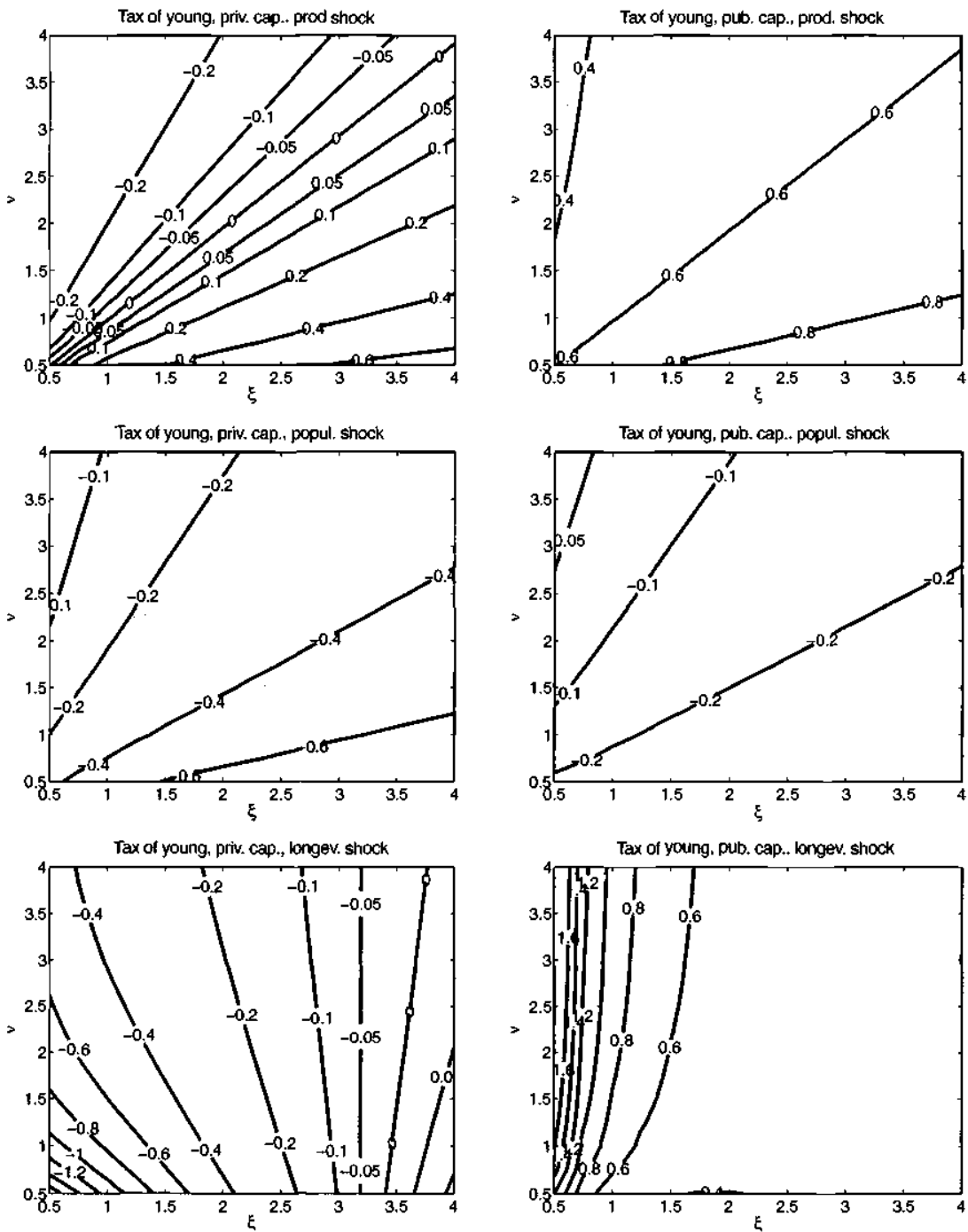

Figure 5.5.

Contour plots. Reaction of total tax payments by young relative to unchanged labor income, as the risk-aversion parameters are varied.

longevity, TFP, labor productivity, population, and depreciation as well as the response of the state variable $s$, as one proceeds from figure 5.6 to 5.11 .

The lower left corner pictures show the persistence effect of shocks due to the endogenous growth feature of the model. However, the ef- 

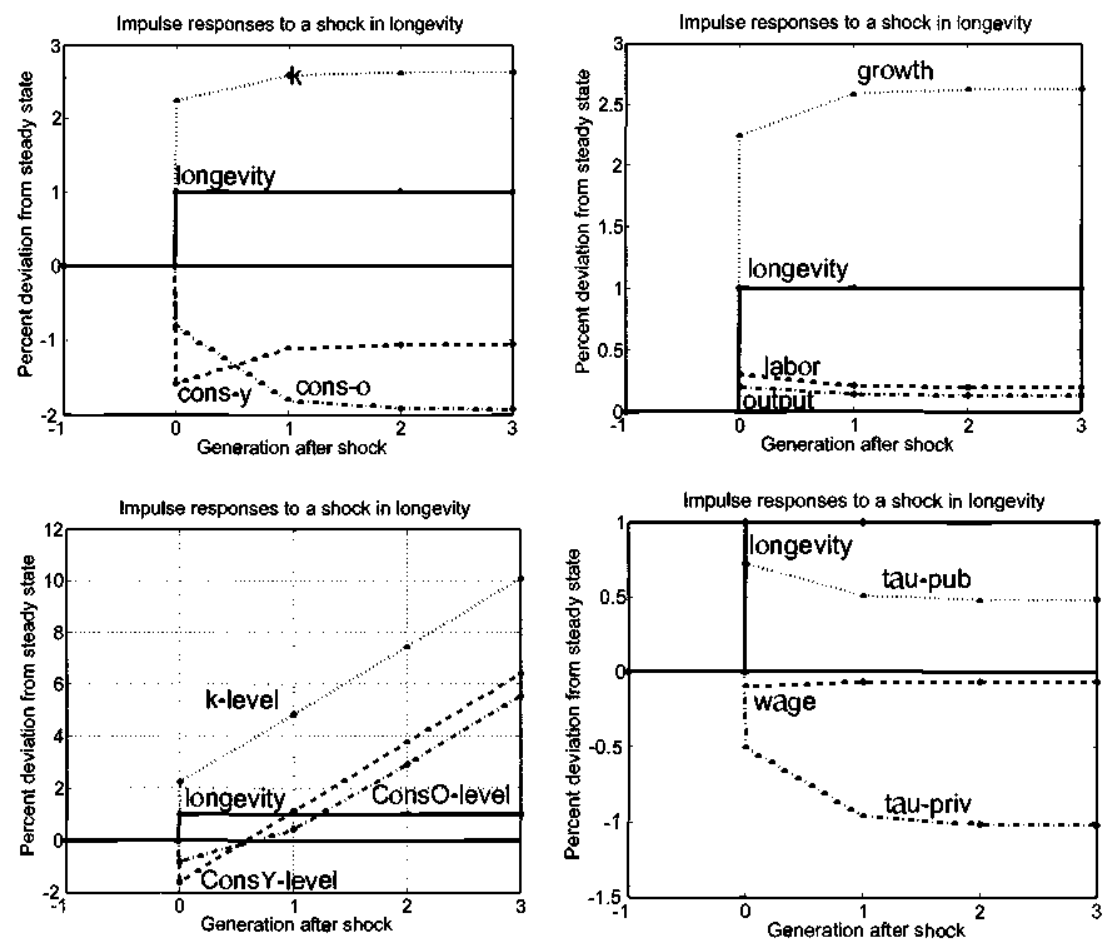

Figure 5.6.

Impulse responses for a 1 percent shock to longevity, given the benchmark parameterization.

fects are usually not an instantaneous adjustment to some new level, but the response is "smeared out" over a few generations, due to the endogenous dynamics of the state variable $s_{t}$. The endogenous dynamics itself is plotted in figure 5.11 .

The responses to a shock in TFP are practically the same (up to scaling) to a shock to labor productivity, because we have essentially assumed a Cobb-Douglas production function. For the benchmark calibration and in reaction, the consumption of the young rises somewhat more than the consumption of the old, which can also be seen by a reduction the young are supposed to make to the pension system, that is, a lowering of their lump-sum taxes. In response to a shock to population growth, consumption falls: essentially, the young now have to make do with less capital per capita than before. Due to the endogenous growth formulation of the model, this effect persists. 

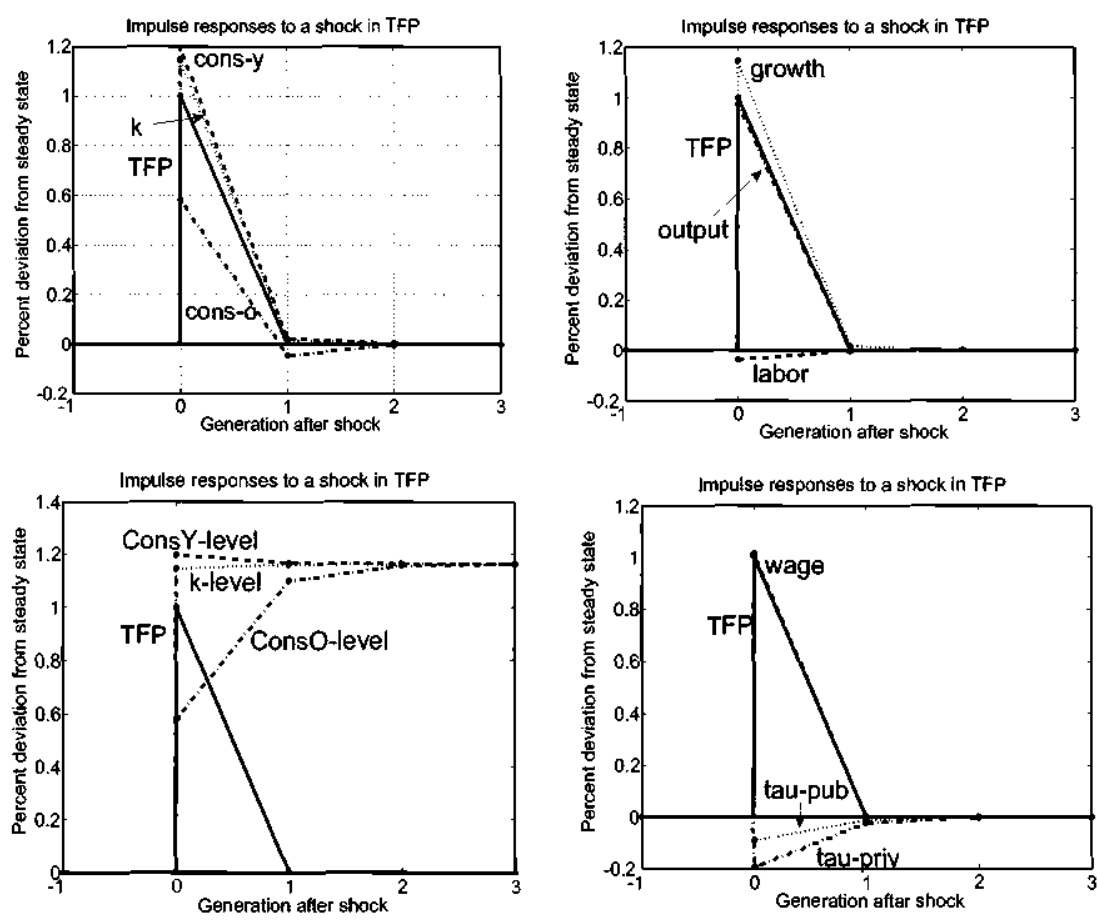

Figure 5.7.

Impulse responses for a 1 percent shock to total factor productivity, given the benchmark parameterization.

\subsubsection{Technology Shocks}

In the simplest risk-sharing case, consumption of the young and old rise by the same percentage (see the first case of the interpretation of the general risk-sharing equation and equation [34]). There, this case is obtained if there is no endogenous labor supply and risk aversion and intertemporal substitution are assumed to be the same for the young and the old, and where there are no life risks. The case of equal risk sharing can arise in the full version of the model as well. Indeed, for the case of a technology shock $Z$ and for the case that $\xi=v$, and with full depreciation $\bar{\delta}=1$, consumption of the young as well as the old rises one for one with productivity, and there is no shift in old-age consumption relative to total consumption, as the first block of table 5.4 shows. The tax payments both for the case of private capital as well as public capital do not react as both labor income (collected by the young) and capital income (collected by the old) rise proportionally with productivity. There is a reaction of the 

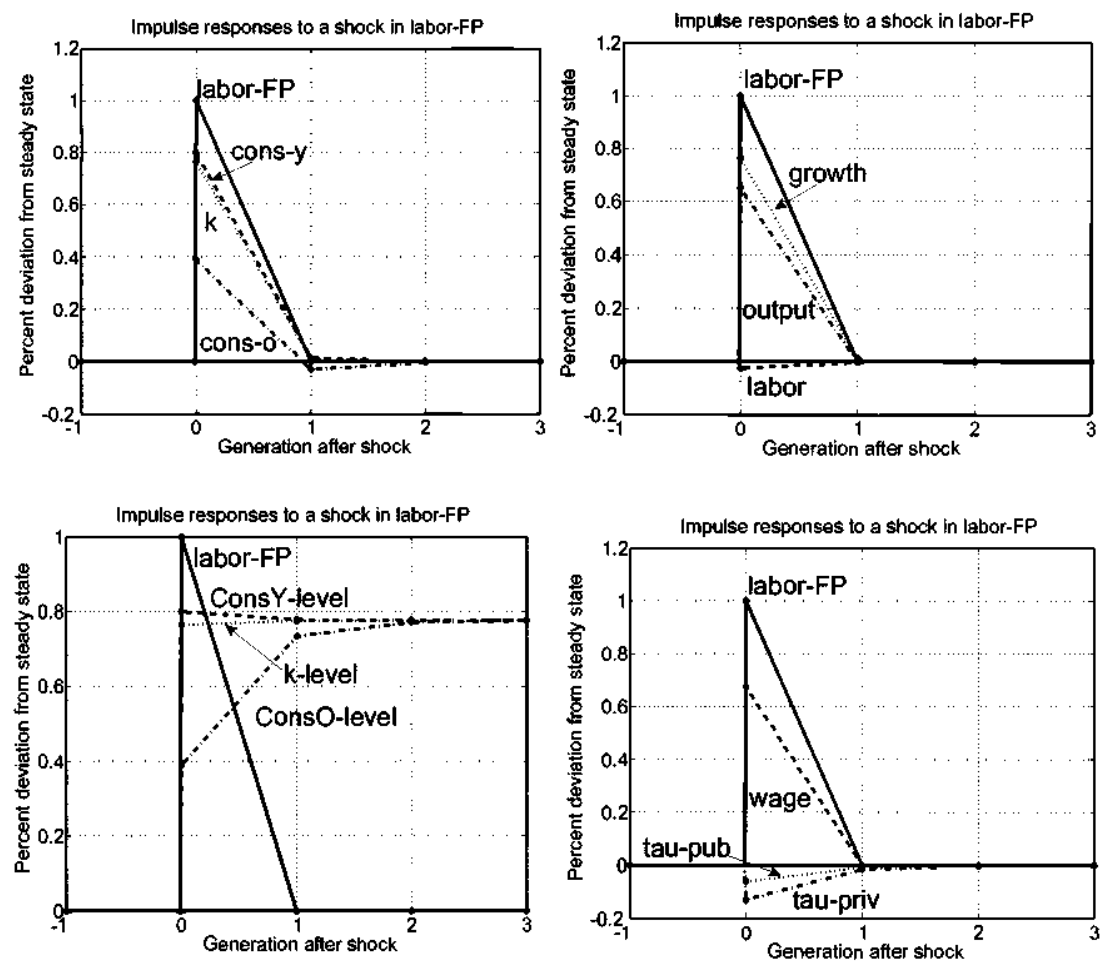

Figure 5.8.

Impulse responses for a 1 percent shock to labor productivity, given the benchmark parameterization.

total tax collection from the young in case of publicly held capital simply because their wages rise, and because the initial tax rate in case of publicly held capital is not zero.

Whether the consumption of the old or the consumption of the young reacts more strongly depends on the ratio between life risk aversion versus risk aversion when old. The upper left corner of the contour plot of figure 5.3 shows a straight line of no reaction in the old-consumption-tototal-consumption ratio for the case $v=\xi$. If $v$ is larger than $\xi$, that is, if the old are relatively more risk averse, then the consumption of the old reacts less strongly, and most of the risk is thus born by the young. Indeed, the young will then consume more goods and consume more leisure (as the income effect dominates the substitution effect), which is why labor declines in response (see the upper right corner of the same contour plot). 

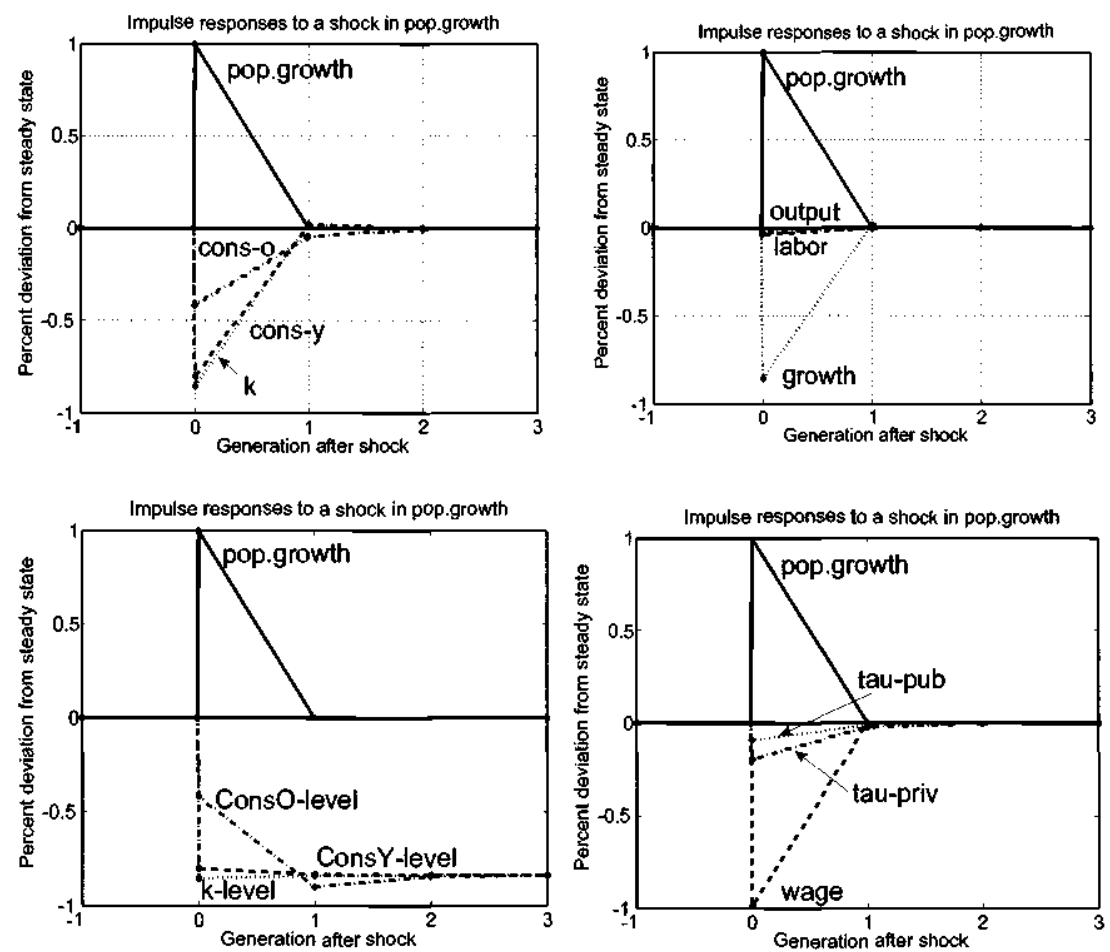

Figure 5.9.

Impulse responses for a 1 percent shock to population growth, given the benchmark parameterization.

To implement the higher sensitivity of the consumption of the young, when the risk aversion of the young is relatively low, actually requires an additional subsidy from the old to the young in the case of privately held capital or a lower total tax payment in the case of publicly held capital if a positive production shock materializes. This is paid out of the increased return to capital in the hands of the old, as, for example, the upper left hand comer of figure 5.4 shows. It is easier to see the intuition for a case of a less-than-expected growth in technology, resulting in lower capital and labor income. In that case, the young have to make up for the low returns on equity by working harder and giving up some of their wage income. In other words, the young bear most of the productivity risk by transferring additional resources to the old in bad times and by collecting additional resources from the old in bad times. Risks are traded in just the opposite way if the old are relatively less risk averse.

The reaction of total consumption is modified by the rate of deprecia- 

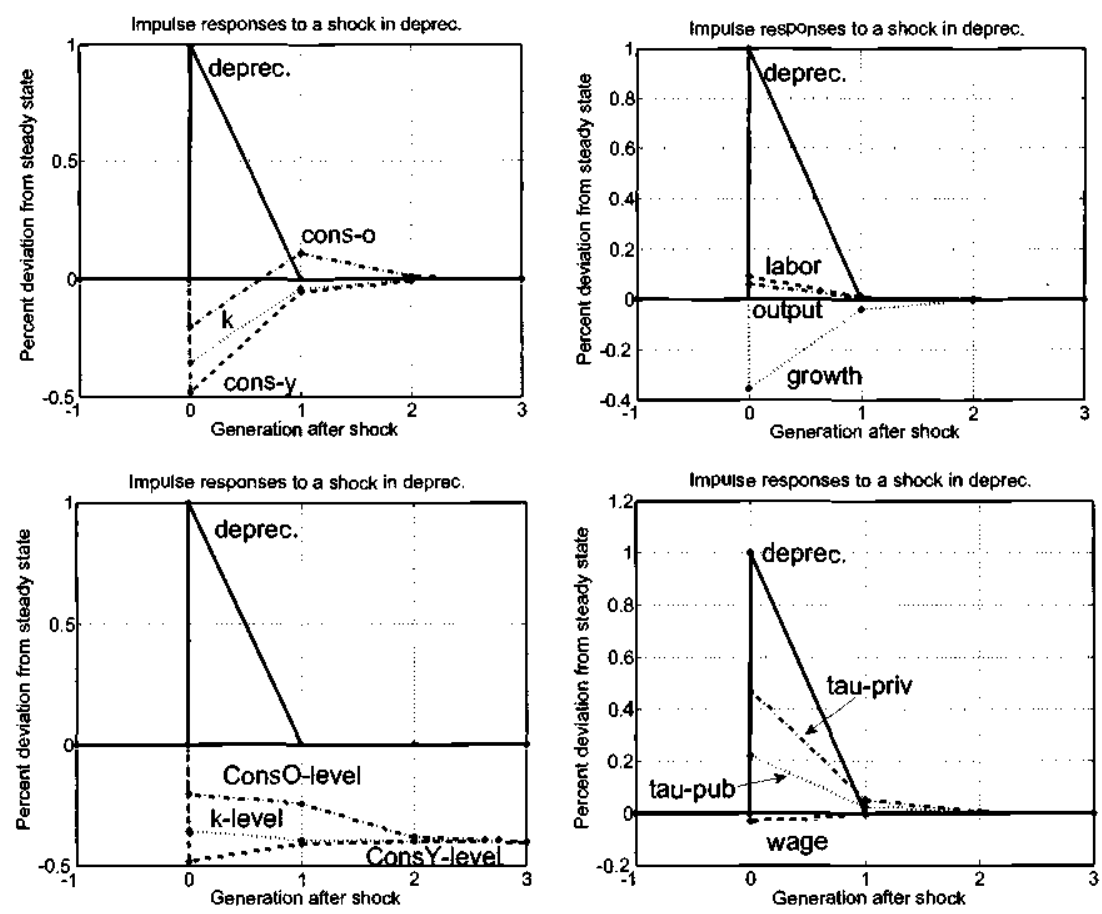

Figure 5.10.

Impulse responses for a 1 percent shock to depreciation, given the benchmark parameterization.

tion: with only partial depreciation, as in the second and fifth column of table 5.4, some consumption smoothing is possible out of capital so production shocks result in less volatile consumption. At the same time, a positive production results in less additional growth (compared to a full 1 percent advantage in growth due to the additional 1 percent growth in TFP).

\subsubsection{Population Growth Shocks}

Note that the consumption of the young and the old always move in the same direction for all shocks, as can be seen both from the impulse responses as well as from table 5.4. This is a key difference between optimal risk sharing and decentralized pension systems. For example, in the absence of insurance, a population growth shock will enhance the return to capital, due to the abundance of labor. At the same time, the 

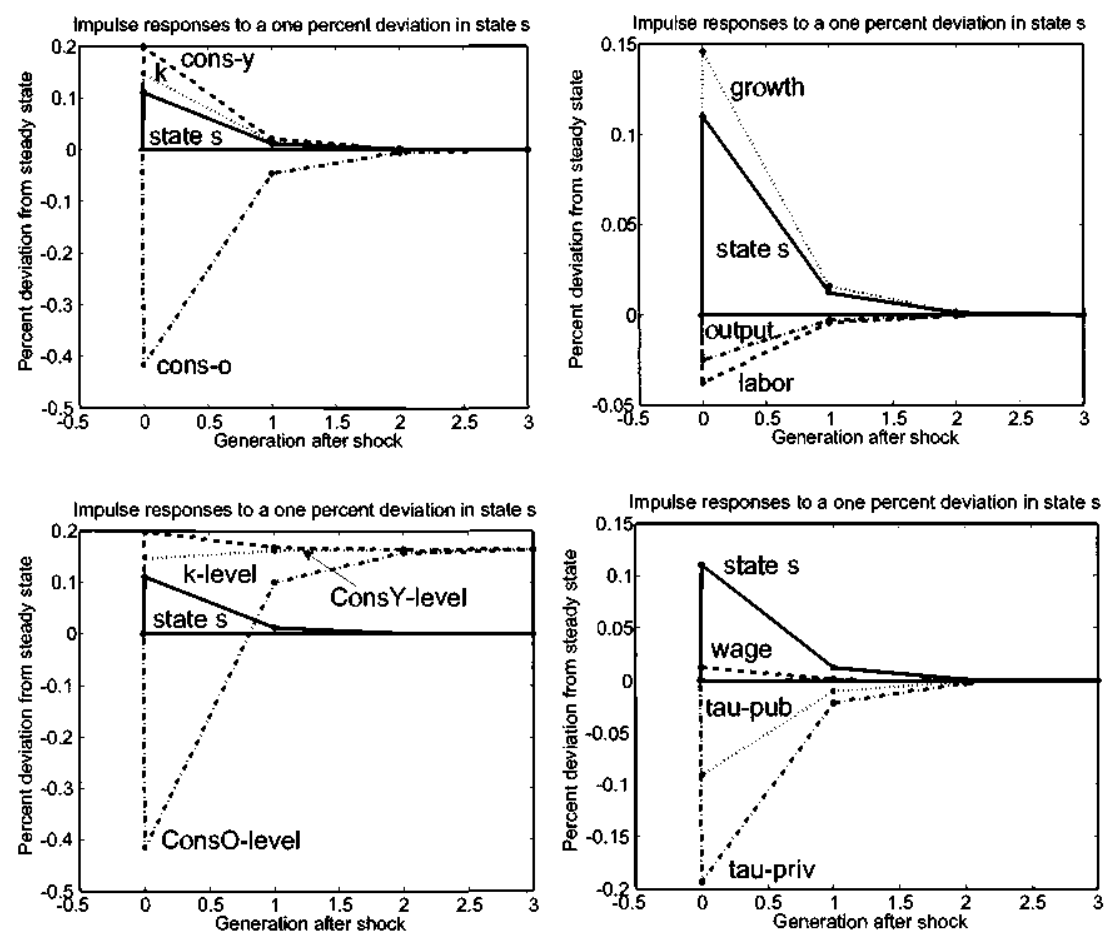

Figure 5.11.

Impulse responses for an initial 1 percent deviation of the state from its steady state.

shock induces lower wages, lower consumption of the young, and higher consumption of the old. This is not the case with optimal risk sharing: both the consumption of the young and the old decrease.

The response of old versus young consumption depends again on the relationship between old-age risk aversion versus life risk aversion. The young need to be actually slightly more risk averse for the relative consumption changes of the old and young to be the same (see the first three columns and the rows for $c_{o}$ and $c_{\text {tot }}$ in table 5.4).

Labor of the young declines with the population shock: this should be an unsurprising consequence of the substitution effect, as the marginal product of labor falls. Note, however, how the income effect (which may lead young agents to work harder) is offset here due to the risk-sharing arrangement with the currently old.

Note also that the difference between the tax rate $\tau_{\text {priv }}$, when not accounting for the changes in wages, population, and labor, and the total tax collection $\tau_{\text {priv,tot }}$ is fairly minor in all cases, that is, even if the steadystate values for these tax rates are nonzero. The same holds true for the 
case of publicly held capital. This result comes about because the additional population growth is almost nearly completely offset by the decline in wages and the change in hours.

\subsubsection{Shocks to Longevity}

The reaction to a longevity shock shows a number of interesting and possibly counterintuitive features. If the young are sufficiently risk neutral, they are asked to work harder individually, in order to somehow generate additional resources. However, as table 5.4 shows, these resources do not go to the currently old-total consumption for the currently old (and therefore, certainly per-period consumption of the currently old) actually falls! Instead, because the longevity shock is persistent, the additional resources are planted into higher capital for the future, and growth picks up. That is, the same generation that now is asked to work harder is also the first generation that gets to enjoy the fruits of this additional labor in the form of higher old-age consumption (see also the impulse response in figure 5.6). The drop in (total) consumption for the currently old is not quite as dramatic as the drop in consumption of the currently young, and thus, consumption is actually shifted in their favor in terms of their share of total consumption.

The intuition for the additional redistribution in favor of future generations in response to increased longevity is as follows. Longer longevity raises the marginal utility of old-age consumption and therefore increases the return on private saving. The higher implicit return on saving makes it more attractive to redistribute toward future generations, especially if the social intertemporal substitution elasticity $1 / \xi$ is large. The comparison between the first three columns and the last three columns of table 5.4 shows indeed that the growth response to longevity is larger if $\xi$ is smaller. We find a larger growth response to an increase in longevity than Bohn (2003) for two reasons. First, our calibration implies a relatively large intertemporal substitution elasticity. Second, in view of the endogenous growth feature of our model, the marginal product of capital does not decline with capital accumulation. Hence, shifting resources over time and between generations can be attractive in response to shocks.

Increased longevity raises not only saving but also work effort. Indeed, the higher marginal utility of consumption on account of a longer expected life makes work more attractive. Note also that the difference between the tax rate $\tau_{\text {priv }}$, when not accounting for the changes in wages, population, and labor, and the total tax collection $\tau_{\text {privtot }}$ is fairly sub- 
stantial if the steady-state values for the tax rates are nonzero, most notably in the fifth column of table 5.4. The same holds true for the case of publicly held capital. This result comes about because the fairly large change in labor supply by the young triggers additional tax collections in that scenario.

\subsection{Conclusion}

We have developed a stochastic endogenous growth with overlapping generations to explore optimal intergenerational risk sharing and redistribution to explore how the pension system can implement optimal intergenerational risk sharing and redistribution between old, young, and future generations. Our endogenous growth model is fairly tractable, despite featuring endogenous labor supply, a number of different shocks, and nonseparable preferences. Depending on the risk aversion of the various generations, the pension system can help to diversify the financial market risks faced by older generations and the labor market and human capital risks faced by younger generations.

A number of interesting insights emerge from the quantitative exploration of the model, as discussed in detail in section 5.6: Neither a defined benefit system or a defined contribution system is typically optimal from a social planner's perspective. In particular, per capita consumption of the young and the old always move in the same direction, even for positive population growth shocks. This result is in contrast to the response of a fully funded decentralized system to such shocks: there, the old would receive higher per capita consumption (due to the increase in return to capital), while the young would receive lower per capita consumption (due to decreasing marginal returns in labor from the larger working population). In contrast to what a defined benefit system offers, longer life expectancy optimally requires old agents to get by with less resources. Indeed, persistent increases in longevity will lead to lower total consumption of the old (and thus certainly lower per-period consumption of the old) and the young as well as to higher work effort of the young. The additional resources are used to increase growth and future output, resulting in higher consumption of future generations.

In future work, we plan to use the model to explore how the intergenerational risk-sharing properties of the pension system affect the equity premium. In particular, in a laissez faire equilibrium without intergenerational risk sharing, the equity premium may be quite high, as the riskaverse retired generations cannot share capital income risk with other 
generations. In the presence of a defined-benefit pension system, however, the old can shed some of this risk to younger generations. In effect, the young issue bonds to the older generations and invest the proceeds in the capital market. In this way, the young have become residual claimants of the pension funds and thus share in the investment risk. By thus spreading investment risk more widely over the population and allowing the young to in effect borrow against their human capital to invest in the stock market (see Constantinides, Donaldson, and Mehra [2002]), the equity premium can fall (and the risk-free rate can rise) in general equilibrium. Indeed, defined-benefit pension funds may help individuals to implement an optimal life investment plan, which typically involves individuals' borrowing heavily in the beginning of their life to invest in the stock market (see Bodie, Merton and Samuelson (1992), Davis, Kubler, and Willen (2002), Jagannathan and Kocherlakota (1996), and Teulings and de Vries [2006]). These pension plans thus in effect allow the young to participate in the stock market. If households can choose between low-risk (and low-return) and high-risk (and highreturn) investments, better intergenerational risk sharing can boost growth by maintaining incentives for risk taking in high-return investments (see Obstfeld [1994] in the context of international risk sharing). At the same time, however, better risk sharing may decrease precautionary saving, thereby reducing capital accumulation and growth. We plan to estimate the potential effects of the pension system in calibrated models.

In allowing intergenerational risk sharing through government intervention, we have assumed that the young cannot participate in capital markets at all to share risks (before the uncertainty during their life time has unfolded). One interpretation is that human capital is not tradable and that the young therefore cannot borrow at all against their human capital to invest in financial capital (see also Constantinides, Donaldson, and Mehra [2002]). In practice, however, the young may be able to participate in equity-market risk that materializes during their working career, either by borrowing, by investing all their saving in the riskbearing capital, or by buying call options. Indeed, capital markets allow in principle for risk sharing between overlapping generations, especially if the young can borrow. In this regard, our calculations, which assumed only two discrete periods of life, are likely to overstate the potential risk-sharing benefits from defined-benefit pension plans. In future work, we would like to explore how sensitive our results are with respect to alternative assumptions about the extent (including the frequency) to which the young can participate in capital markets. 
Our analysis has assumed that governments can implement optimal intergenerational risk sharing by committing future generations to a risk-sharing contract. For the question of implementability, additional aspects seem crucial. First, government intervention may not only help to share market risks but also give rise to new risks. These additional political risks must be traded off against the possible gains in sharing market risks. Second, the government may face serious problems in committing future generations to an optimal complete risk-sharing contract. For one, in a democracy in which current generations have the voting power, the government faces a serious commitment problem; future generations can always opt out. For another, the government faces substantial fundamental uncertainty, so that complete contracts are excessively costly. With substantial fundamental uncertainty, discretion rather than rules become optimal.

Optimal risk sharing is sensitive to the magnitude of ex ante risk aversion $\xi$ versus ex post risk aversion $\nu$. The latter type of risk aversion may exceed ex ante risk aversion in the presence of habit formation (see also Bohn [2003]). Habit formation, however, gives rise to new phenomena. Another explanation for high levels of risk aversion is the standard-ofliving utility, in which people are sensitive to their utility level compared to that of others. Exploring the sensitivity of our results to alternative specifications of preferences is an important subject for future research.

We have relied on numerical solutions. In some special cases, however, we can solve the model analytically. This may provide a useful benchmark for understanding the features of the optimal solution in other, more complicated cases. We plan to investigate the analytical solutions in the future.

\section{Acknowledgments}

This research was supported by the Deutsche Forschungsgemeinschaft through the SFB 649 "Economic Risk." We are grateful to Christian Stoltenberg for his research assistance.

\section{Notes}

1. Note that the production side is already made scale invariant by construction, that is, our assumption about AK-type endogenous growth.

2. This is shown in the Appendix. 


\section{References}

Barbie, Martin, Marcus Hagedorn, and Ashok Kaul. 2004. Assessing aggregate tests of efficiency for dynamic economies. Topics in Macroeconomics 4(1): Article 16, http: / www.bepress.com/bejm/topics/vol4/issl/artl6.

2004. On the feasibility of debt ponzi schemes-A bond portfolio approach: Theory and some evidence. Econometric Society Working Paper no. 541. New York: The Econometric Society.

Blanchard, Olivier, and Philippe Weil. 2001. Dynamic efficiency, the riskless rate and debt ponzi games under uncertainty. MIT Department of Economics Working Paper no. 01-41. Cambridge, MA: MIT.

Bodie, Zvi, Robert C. Merton, and William F. Samuelson. 1992. Labor supply flexibility and portfolio choice in a life cycle model. Journal of Economic Dynamics and Control 16(3-4): 427-49.

Bohn, Henning. 1998. Risk sharing in a stochastic overlapping generations economy. Department of Economics, Working Paper no. 3-98. Santa Barbara: University of California at Santa Barbara.

1999. Social Security and demographic uncertainty: The risk sharing properties of alternative policies. NBER Working Paper no. 7030. Cambridge, MA: National Bureau of Economic Research.

- 2003. Intergenerational risk sharing and fiscal policy. Working paper, Department of Economics, University of California at Santa Barbara.

2005. Who bears what risk? An intergenerational perspective. Pension Research Council Working Paper no. 2005-7. Philadelphia: University of Pennsylvania.

Canton, Erik. 1997. Economic growth and business cycles. PhD diss., Tilburg University.

. 2002. Business cycles in a two-sector model of endogenous growth. Economic Theory $19(3): 477-92$.

Conesa, J. C., and Dirk Krueger. 1999. Social security reform with heterogeneous agents. Review of Economic Dynamics 2(4): 757-95.

Constantinides, George M., John B. Donaldson, and Rajnish Mehra. 2002. Junior can't borrow: A new perspective on the equity premium puzzle. Quarterly Journal of Economics 117(1): 269-96.

Davis, Steven J., Felix Kubler, and Paul Willen. 2006. Borrowing costs and the demand for Equity over the life cycle. The Review of Economics and Statistics 88(2): 348-62.

Demange, Gabrielle. 2002. On optimality in intergenerational risk sharing. Economic Theory 20(1): 1-27.

De Menil, Georges, Fabrice Murtin, and Eytan Sheshinski. 2006. Planning for the optimal mix of pay-go tax and funded savings. Journal of Pension Economics and Finance 5(1): 1-25.

Diamond, Peter. 1965. National debt in a neoclassicial growth model. American Economic Review 55(5): 1126-50.

Epstein, Larry G., and Stanley E. Zin. 1989. Substitution, risk aversion and the temporal behavior of consumption and asset returns: A theoretical framework. Econometrica 57(4): $937-69$. 
Gordon, Roger H., and Hal R. Varian. 1988. Intergenerational risk sharing. Journal of Public Economics 37(2): 185-202.

Hall, Robert E., and Charles I. Jones. 2004. The value of life and the rise in health spending. NBER Working Paper no. 10737. Cambridge, MA. National Bureau of Economic Research.

Jagannathan, Ravi, and Narayana Kocherlakota. 1996. Why should older people invest less in stocks than younger people? Federal Reserve Bank of Minneapolis Quarterly Review 20(3): 11-23.

Judd, Kenneth L., and Sy-Ming Guu. 2001. Asymptotic methods for asset market equilibrium analysis. Economic Theory 18(1): 127-57.

King, Robert, Charles I. Plosser, and Sergio T. Rabelo. 1988. Production, growth, and business cycles: I. The basic neoclassical model. Journal of Monetary Economics 21:195-232.

2002. Production, growth and business cycles: technical Appendix. Computational Economics 20(1-2): 87-116.

Krueger, D., and F. Kubler. 2002. Intergenerational risk sharing via Social Security when financial markets are incomplete. American Economic Review 92(2): 407-10.

- 2004. Computing equilibrium in OLG models with stochastic production. Journal of Economic Dynamics and Control 28(7): 1411-36.

2006. Pareto improving Social Security when financial markets are incomplete. American Economic Review 96(3): 737-55.

Lindbeck, Assar, and Mats Persson. 2003. The gains from pension reform. Journal of Economic Literature 41(1): 74-112.

Merton, R. 1983. On the role of Social Security as a means for efficient risk sharing in an economy where human capital is not tradable. In Financial aspects of the United States pension system, ed. Z. Brodie and J. Shoven, 325-58. Chicago: University of Chicago Press.

Murphy, Kevin M., and Robert Topel. 2003. The economic value of medical research. In Kevin M. Murphy and Robert H. Topel, eds., Exceptional returns. Chicago: University of Chicago Press.

Obstfeld, Maurice. 1994. Risk-taking, global diversification and growth. American Economic Review 84(5): 1310-29.

Olovsson, Conny. 2004. The welfare gains of improved risk sharing in Social Security. Institute for International Economic Studies Seminar Paper no. 728. Stockholm: Institute for International Economic Studies.

Samuelson, Paul A. 1970. The fundamental approximation theorem of portfolio analysis in terms of means, variances and higher moments. Review of Economic Studies 37(4): 537-42.

Shiller, Robert J. 1999. Social Security and institutions for intergenerational, intragenerational, and international risksharing. Carnegie-Rochester Conference Series on Public Policy 50:165-204.

Teulings, Coen N., and Casper G. de Vries. 2003. Generational accounting and portfolio management for pension funds. Unpublished manuscript. The Netherlands. Erasmus Universiteit Rotterdam and Tinbergen Institute.

Uhlig, Harald. 1999. A toolkit for analysing nonlinear dynamic stochastic models easily. In Computational methods for the study of dynamic economies, ed. Ramon Marimon and Andrew Scott, 30-61. Oxford, UK: Oxford University Press. 
Weil, Philippe. 1990. Nonexpected utility in macroeconomics. Quarterly Journal of Economics 105(1): 29-42.

Yanagawa, Noriyuki, and Harald Uhlig. 1996. Increasing the capital income tax may lead to faster growth. European Economic Review 40(8): 1521-40.

\section{Appendix A Details and Additionally Useful Results}

\section{A.1 The Calculations for Proposition 2}

The explicit expressions for the logarithmic derivatives are

$$
\begin{aligned}
& \ell_{u, c, t}=\frac{u_{c}\left(\bar{c}_{t, y}, \bar{n}_{t}\right) \bar{c}_{t, y}}{u\left(\bar{c}_{t, y}, \bar{n}_{t}\right)}, \\
& \ell_{t, n, t}=\frac{u_{n t}\left(\bar{c}_{t, y}, \bar{n}_{t}\right) \bar{n}_{t}}{u\left(\bar{c}_{t, y}, \bar{n}_{t}\right)}, \\
& \ell_{x, t+1}=\frac{x^{\prime}\left[\overline{\boldsymbol{\varpi}}_{t+1} q\left(\frac{\bar{c}_{t+1, o}}{\overline{\boldsymbol{\varpi}}_{t+1}}\right)\right] \overline{\boldsymbol{\varpi}}_{t+1} q\left(\frac{\bar{c}_{t+1, o}}{\overline{\boldsymbol{\varpi}}_{t+1}}\right)}{x\left[\overline{\boldsymbol{\varpi}}_{t+1} q\left(\frac{\bar{c}_{t+1,0}}{\overline{\boldsymbol{\varpi}}_{t+1}}\right)\right]} \\
& \ell_{q, t+1}=\frac{q^{\prime}\left(\frac{\bar{c}_{t+1, o}}{\overline{\boldsymbol{\sigma}}_{t+1}}\right) \frac{\bar{c}_{t+1,0}}{\overline{\boldsymbol{\sigma}}_{t+1}}}{q\left(\frac{\bar{c}_{t+1,0}}{\overline{\boldsymbol{\sigma}}_{t+1}}\right)},
\end{aligned}
$$

Furthermore, define

$$
\begin{aligned}
\mu_{z, t} & =-\frac{z^{\prime \prime}\left(\bar{a}_{t}\right) \bar{a}_{t}}{z^{\prime}\left(\bar{a}_{t}\right)}, \\
\mu_{u c, c, t} & \left.=-\frac{u_{c c}\left(\bar{c}_{t, y^{\prime}} \bar{n}_{t}\right) \bar{c}_{t, y}}{u_{c}\left(\bar{c}_{t, y^{\prime}}\right.} \bar{n}_{t}\right) \\
\mu_{q, t} & =-\frac{q^{\prime \prime}\left(\frac{\left(\bar{c}_{t, o}\right.}{\overline{\boldsymbol{\sigma}}_{t}}\right) \frac{\bar{c}_{t, o}}{\overline{\boldsymbol{\sigma}}_{t}}}{q^{\prime}\left(\frac{\bar{c}_{t, o}}{\overline{\boldsymbol{\sigma}}_{t}}\right)},
\end{aligned}
$$




$$
\mu_{x, t}=-\frac{x^{\prime \prime}\left[\overline{\boldsymbol{\varpi}}_{i} q\left(\frac{\bar{c}_{t, o}}{\overline{\boldsymbol{\varpi}}_{t}}\right)\right] \overline{\boldsymbol{\varpi}}_{t} q\left(\frac{\bar{c}_{t, o}}{\overline{\boldsymbol{\varpi}}_{t}}\right)}{x^{\prime}\left[\overline{\boldsymbol{\varpi}}_{t} q\left(\frac{\bar{c}_{t, o}}{\overline{\boldsymbol{\varpi}}_{t}}\right)\right]}
$$

$\mu_{u c, n, t}=-\frac{u_{n c}\left(\bar{c}_{t, y}, \bar{n}_{t}\right) \bar{n}_{t}}{u_{c}\left(\bar{c}_{t, y}, \bar{n}_{t}\right)}$.

Recall (30),

$\alpha_{t}=\frac{u\left(\bar{c}_{t, y}, \bar{n}_{t}\right)}{\bar{a}_{t}}$.

Note that all these logarithmic derivatives are known as of $t-1$ by assumption. The time subscript therefore does not indicate "information", but rather indicates the argument.

To derive the equations in proposition 2, one needs to take a firstorder Taylor expansion in the logarithmic deviations. These calculations can often be performed rather speedily by combining several "rules", which are easily verified:

[Rule 1:] To take the Taylor expansion for a single variable, note $x_{t}=\bar{x}_{t} \exp \check{x}_{t} \approx \bar{x}_{t}\left(1+\hat{x}_{t}\right)$.

[Rule 2:] If $z_{t}=A x_{t} y_{t}$, where $A$ is some constant, and the same is true for the benchmark values, then

$\check{z}_{t}=\check{x}_{t}+\check{y}_{t}$.

[Rule 3:] If $z_{t}=x_{t}+y_{t}$ and the same is true for the benchmark values, then

$\bar{z}_{t} \check{z}_{t}=\bar{x}_{t} \check{x}_{t}+\bar{y}_{t} \check{y}_{t}$

[Rule 4:] Suppose some variable $z_{t}$ satisfies $z_{t}=f\left(x_{t}\right)$ as well as $\bar{z}_{t}=f\left(\bar{x}_{t}\right)$. Then,

$\check{z}_{t}=\ell_{f} \check{x}_{t}$.

where $\ell_{f}$ is the logarithmic derivative of $f(\cdot)$ at $\bar{x}_{t}$, $\ell_{f}=\frac{f^{\prime}\left(\bar{x}_{t}\right) \bar{x}_{t}}{f\left(\bar{x}_{t}\right)}$. 
The extension to two variables, $z_{t}=f\left(x_{t}, y_{t}\right)$ is

$\check{z}_{t}=\ell_{f, x} \check{x}_{t}+\ell_{f, y} \check{y}_{t}$

where

$\ell_{f, x}=\frac{f_{x}\left(\bar{x}_{t}, \bar{y}_{t}\right) \bar{x}_{t}}{f\left(\bar{x}_{t}, \bar{y}_{i}\right)}, \ell_{f, y}=\frac{f_{y}\left(\bar{x}_{t}, \bar{y}_{i}\right) \bar{y}_{t}}{f\left(\bar{x}_{t}, \bar{y}_{t}\right)}$.

Note furthermore, that

$\ell_{x^{-1, \ell}}=\ell_{x, t+1}^{-1}$

and that

$\mu_{x^{-1, t}}=-\frac{\mu_{x, t+1}}{\ell_{x, t+1}}$

Define the variables

$$
\begin{aligned}
z_{t}^{\prime} & \equiv z^{\prime}\left(a_{t}\right) \\
u_{t} & \equiv u\left(c_{t, y^{\prime}} n_{t}\right) \\
u_{c, t}^{\prime} & \equiv \frac{\partial u\left(c_{t, y^{\prime}} n_{t}\right)}{\partial c_{t, y}} \\
x_{t} & \equiv x\left[\varpi_{t} q\left(\frac{c_{t, o}}{\varpi_{t}}\right)\right] \\
x_{t}^{\prime} & \equiv x^{\prime}\left[\varpi_{t} q\left(\frac{c_{t, o}}{\varpi_{t}}\right)\right] \\
e_{t} & \equiv E_{t}\left[x_{t+1}\right] \\
\iota_{t} & \equiv x^{-1}\left(e_{t}\right) \\
\iota_{t-1}^{\prime} & \equiv\left(x^{-1}\right)^{\prime}\left(e_{t-1}\right) \\
q_{t}^{\prime} & \equiv q^{\prime}\left(\frac{c_{t, o}}{\varpi_{t}}\right)
\end{aligned}
$$

Note that $\vec{e}_{t}=x\left[\overline{\boldsymbol{\omega}}_{t+1} q\left(\bar{c}_{t+1, o} / \overline{\boldsymbol{\omega}}_{t+1}\right)\right]$ and $\bar{e}_{t-1}=x\left[\overline{\boldsymbol{\omega}}_{t} q\left(\bar{c}_{t, o} / \overline{\boldsymbol{\omega}}_{t}\right)\right]$, since we assume that the benchmark levels are known at date $t-1$ already.

With this, the risk-sharing condition (29),

$$
z^{\prime}\left(a_{t}\right) \frac{\partial u\left(c_{t, y} n_{t}\right)}{\partial c_{t, y}}=z^{\prime}\left(a_{t-1}\right) \frac{\beta}{\omega} d_{t} q^{\prime}\left(\frac{c_{t, o}}{\varpi_{t}}\right)
$$


can be written as

$z_{t}^{\prime} u_{c, t}^{\prime}=z_{t-1}^{\prime} \frac{\omega}{\beta} d_{t} q_{t}^{\prime}$

The argument definition

$a_{t}=u\left(c_{t, y}, n_{t}\right)+\beta x^{-1}\left(E_{t}\left\{x\left[\varpi_{t+1} q\left(\frac{c_{t+1, o}}{\varpi_{t+1}}\right)\right]\right\}\right)$

can likewise be written as

$a_{t}=u_{t}+\beta \iota_{t}$

Finally, the definition for the discounting correction (20),

$d_{t}=\left(x^{-1}\right)^{\prime}\left(E_{t-1}\left\{x\left[\varpi_{t} q\left(\frac{c_{t, o}}{\boldsymbol{\varpi}_{t}}\right)\right]\right\}\right) x^{\prime}\left[\varpi_{t} q\left(\frac{c_{t, o}}{\boldsymbol{\varpi}_{t}}\right)\right]$,

can be written as

$d_{t}=\iota_{t-1}^{\prime} x_{t}^{\prime}$.

1. Taking logarithms of equation (79) delivers

$\check{z}_{t}^{\prime}+\check{u}_{c, t}^{\prime}=\check{z}_{t-1}^{\prime}+\check{d}_{t}+\check{q}_{t}^{\prime}$.

Application of rule 4 to these items immediately delivers (84),

$\boldsymbol{\mu}_{z, t} \check{a}_{t}+\mu_{t c, c, t} \check{c}_{t, y}+\mu_{u c, n, t} \check{n}_{t}=\mu_{z, t-1} \check{a}_{t-1}+\check{d}_{t}+\mu_{q, t}\left(\check{c}_{t, o}-\check{\varpi}_{t}\right)$.

2. To loglinearize (81), use rule 3 to obtain

$\check{a}_{t}=\alpha_{t} \check{u}_{t}+\left(1-\alpha_{t}\right) \check{\mathrm{l}}_{t}$.

With rule 4, extended to two arguments, and applied to $u_{t}=u(c, n)$, $\check{u}_{t}=\ell_{u, c, t} \check{c}_{t, y}+\ell_{u, n, t} \check{n}_{t}$.

Also with rule 4 ,

$\check{\mathrm{i}}_{\mathrm{t}}=\ell_{i, t} \check{e}_{t}$.

Interchanging expectation and differentiation, rule 4 delivers

$\check{e}_{t}=\ell_{x, t+1}\left[\left(1-\ell_{q, t+1}\right) E_{t}\left(\check{\boldsymbol{w}}_{t+1}\right)+\ell_{q, t+1} E_{t}\left(\check{c}_{t+1, o}\right)\right]$.

Combining and exploiting (77), one obtains equation (33), i.e.

$$
\begin{aligned}
\check{a}_{t}= & \alpha_{t}\left(\ell_{u t, c t} \check{c}_{t, y}+\ell_{u, n_{t}} \check{n}_{t}\right) \\
& +\left(1-\alpha_{t}\right)\left[\left(1-\ell_{q, t+1}\right) E_{t}\left(\check{\varpi}_{t+1}\right)+\ell_{q, t+1} E_{t}\left(\check{c}_{t+1, o}\right)\right] .
\end{aligned}
$$


3. Taking logarithms of (83) delivers

$\check{d}_{t}=\check{\mathfrak{t}}_{t-1}^{\prime}+\check{x}_{t}^{\prime}$.

For $\check{x}_{r}^{\prime}$, rule 4 delivers

$\check{x}_{t}^{\prime}=-\mu_{x, t}\left[\left(1-\ell_{q, t}\right) \check{\varpi}_{t}+\ell_{q, t} \check{c}_{t, o}\right]$.

For $\check{c}_{t-1}^{\prime}$, repeated application of rule 4 delivers

$\check{\iota}_{t-1}^{\prime}=-\mu_{x^{-1, t-1}} \check{e}_{t}-\mu_{x^{-1, t-1}} E_{t-1}\left\{\ell_{x, t}\left[\left(1-\ell_{q, t}\right) \check{\varpi}_{t}+\ell_{q, t} \check{c}_{t, 0}\right]\right\}$.

Combining and exploiting (78) delivers equation (33), i.e.

$\check{d}_{t}=\mu_{x, t}\left\{\left(1-\ell_{q, t}\right)\left[\check{\varpi}_{t}-E_{t-1}\left(\check{\boldsymbol{v}}_{t}\right)\right]+\ell_{q, i}\left[\check{c}_{t, o}-E_{t-1}\left(\check{c}_{t, o}\right)\right]\right\}$

\section{A.2 Scale Invariance and Balanced Growth}

Scale invariance has a number of implications.

1. To save on notation, define $\check{c}_{t, y}=\phi c_{t, y}, \check{c}_{t, 0}=\phi c_{t, 0}$ and $\check{w}_{t}=\phi w_{t}$ : these are part of the allocation $\breve{\Phi}$. Thus, equation (22) can be rewritten as

$\frac{\ell_{u, c, t}}{\ell_{u, n, t}}=-\frac{c_{t, y}}{w_{t} n_{t}}$

Note that the right-hand side-which can be interpreted as the income share spend on consumption of the young-does not depend on $\phi$. Hence, the ratio of the two logarithmic derivatives $\ell_{u, c, t}=\ell_{u, c, t}\left(\breve{c}_{t, y}, n_{t}\right)$ and $\ell_{t, n, t}=\ell_{u, n, t}\left(\check{c}_{t, y}, n_{t}\right)$ is independent of the argument $\check{c}_{t, y}$.

2. Suppose that the income share spend on consumption of the young is constant

$\frac{c_{t, y}}{w_{t} n_{t}} \equiv \sigma_{y^{\prime}}$

then

$\ell_{u, c, t}=\sigma_{y} \ell_{u, n, t}$.

We note that this condition will hold along a balanced growth path, where indeed the income share should be constant.

3. Replace $c_{t, y}$ with $\phi c_{t, y}$ in (22), and take the logarithmic derivative with respect to $\phi$ to obtain

$0=-\mu_{u c, c, t}-\mu_{u c, n, t} \frac{c_{t, y}}{w_{t} n_{t}}+1$, 
or

$0=-\mu_{u c, c, t}+\mu_{u c, n, t} \frac{\ell_{u, c, t}}{\ell_{u, n, t}}+1$

across all values $\tilde{c}_{t, y}$.

4. Suppose additionally, that $\mu_{u c, c, t}$ is constant over time and $\eta \equiv \mu_{u c, c, t}$. We can derive a semiclosed form for the utility function $u(c, n)$, as is wellknown from the literature (see, e.g., King and Plosser [1989]). We provide the derivation here for the sake of completeness. Consider first the case $\eta \neq 1$. Since $\mu_{u c, c, t}$ is the (negative) logarithmic derivative of $u_{c}$ with respect to $c$, this implies $\log$-linearity for $u_{c}$,

$\log u_{c}\left(\breve{c}_{t, y}, n_{t}\right)=(1-\eta) \log v\left(n_{t}\right)-\eta \log \breve{c}_{t, y}$

where the "constant" term $(1-\eta) \log v\left(n_{t}\right)$, may depend on $n_{t}$ (and seems to have been written in a rather complicated fashion, as this will turn out to be convenient later). Rewrite as

$u_{c}\left(\breve{c}_{t, y}, n_{t}\right)=v\left(n_{t}\right)^{1-\eta} \breve{c}_{t, y}^{-\eta}$,

and integrate to obtain

$u\left(\breve{c}_{t, y}, n_{t}\right)=\frac{\left[v\left(n_{t}\right) \breve{c}_{t, y}\right]^{1-\eta}}{1-\eta}+\kappa\left(n_{t}\right)$

where $\kappa\left(n_{t}\right)$ is an additional constant, possibly depending on $n_{t}$. Differentiating with respect to $n_{t}$ and comparing to (22) shows that $\mathrm{\kappa}\left(n_{t}\right) \equiv \kappa$ independent of $n_{t}$ as follows. Rewrite (22) for the allocation $\breve{\Phi}$ as

$$
\begin{aligned}
\phi w_{t} & \left.=-\frac{u_{n}\left(\phi c_{t, y^{\prime}} n_{t}\right)}{u_{c}\left(\phi c_{t, y^{\prime}}\right.} n_{t}\right) \\
& =-\frac{v^{\prime}\left(n_{t}\right)}{v\left(n_{t}\right)} \phi c_{t, y}-\frac{\kappa^{\prime}\left(n_{t}\right)}{v\left(n_{t}\right)^{1-\eta}\left(\phi c_{t, y}\right)^{-\eta}} .
\end{aligned}
$$

Divide by $\phi$ to see that the left-hand side and the first term on the righthand side do not depend on $\phi$, whereas the last one does, unless either $\eta=1$ or $\kappa^{\prime}\left(n_{t}\right)=0$. Since we assumed $\eta \neq 1, \kappa\left(n_{t}\right)$ must be a constant, independent of $n_{i}$. The case $\eta=1$ similarly yields

$u\left(\breve{c}_{t, y}, n_{t}\right)=\log \breve{c}_{t, y}+v\left(n_{t}\right)$.

5. Consider a nonstochastic case. Equation (26) reads 


$$
\begin{aligned}
u_{c}\left(\phi c_{t, y^{\prime}} n_{t}\right)= & \beta\left(x^{-1}\right)^{\prime}\left\{x\left[\varpi_{t+1} q\left(\frac{\phi c_{t+1, o}}{\varpi_{t+1}}\right)\right]\right\} \\
x^{\prime} & {\left[\varpi_{t+1} q\left(\frac{\phi c_{t+\mathbf{1}, o}}{\varpi_{t+1}}\right)\right] q^{\prime}\left(\frac{\phi c_{t+\mathbf{1}, o}}{\varpi_{t+1}}\right) R_{t+1} . }
\end{aligned}
$$

Take the logarithmic derivative with respect to $\phi$ and exploit (78) to find $\mu_{u c, c, t}=\mu_{q, t+1}$.

6. Again for the nonstochastic case, the constant share condition $\tilde{\alpha}_{t}=\alpha_{t}$ can be rewritten as

$\left(\frac{1}{\alpha_{\imath}}-1\right) u\left(\phi c_{t, y}, n_{\imath}\right)=\beta q\left(\phi c_{t+1, o}\right)$

where $\alpha_{t}$ does not depend on $\phi$. Taking the logarithmic derivative with respect to $\phi$, we establish

$\ell_{u, \mathcal{L}, t}=\ell_{q, t+1}$.

\section{A.3 The Equity Premium}

To relate the risk-aversion parameters to observables, one possibility is that observed allocations are (nearly) optimal as far as intergenerational risk sharing is concerned, and to calibrate the risk-aversion parameters to observed market prices of risk. In our context, this means to observe market prices of risk from young agents trading assets that pay off when they are old.

Given an allocation which solves the social planner's problem, suppose that the social planner contemplates transferring resources between periods using some asset $a$ with return $R_{t+1}^{a}$. Since the allocation is already optimal, the social planner must wish not to execute this reallocation. Hence, it has to be the case that the asset pricing condition (26) not only holds for $R_{t+1}$, but for the return $R_{t+1}^{a}$ as well, which might, of course, coincide with $R_{t+1}$. Recall the definition of the stochastic discount factor $m_{t+1}$,

$$
\begin{gathered}
m_{t+1}=\beta\left(x^{-1}\right)^{\prime}\left(E_{t}\left\{x\left[\varpi_{t+1} q\left(\frac{c_{t+1, o}}{\varpi_{t+1}}\right)\right]\right\}\right) \\
x^{\prime}\left[\varpi_{t+1} q\left(\frac{c_{t+1, o}}{\varpi_{t+1}}\right)\right] q^{\prime}\left(\frac{c_{t+1, o}}{\varpi_{t+1}}\right)
\end{gathered}
$$


The asset pricing equation (26) then implies

$E_{t}\left(m_{t+1} R_{t+1}^{a}\right)=E_{t}\left(m_{t+1} R_{t+1}\right)$.

Decompose $R_{t+1}^{a}, c_{t+1, o}, \varpi_{t+1}, m_{s, t+1}$ into their predicted part and surprise part,

$\log \left(R_{t+1}^{a}\right)=\log \left(\bar{R}_{t+1}^{a}\right)+\check{R}_{t+1}^{a}$,

$\log \left(c_{t+1,0}\right)=\log \left(\bar{c}_{t+1,0}\right)+\check{c}_{t+1}$

$\log \left(\varpi_{t+1}\right)=\log \left(\bar{\varpi}_{t+1}\right)+\check{\varpi}_{t+1}$,

$\log \left(m_{t+1}\right)=\log \left(\bar{m}_{t+1}\right)+\check{m}_{t+1}$,

with

$0=E_{t}\left(\check{R}_{t+1}^{a}\right)=E_{t}\left(\check{c}_{t+1}\right)=E_{t}\left(\check{\varpi}_{t+1}\right)=E_{t}\left(\check{m}_{t+1}\right)$,

We assume that the surprise parts are jointly normally distributed, conditional on information up to and including $t$. Denote the standard deviations by, for example, $\sigma_{R, t+1}$ and $\sigma_{m, t+1}$ and correlations denoted by, for example, $\rho_{m, R, t+1}$. Define the Sharpe ratio, defined as the difference of the log expected returns divided by the standard deviation of the log return.

$\mathbf{S R}_{t}=\frac{\log \left[E_{t}\left(R_{t+1}\right)\right]-\log \left(R_{t}^{f}\right)}{\sigma_{R, t+1}}$.

Using a standard calculation (see Lettau and Uhlig [2002]), one can show that

$\mathrm{SR}_{t}=-\rho_{m, R, t+1} \sigma_{m, t+1}$

It is easy to see that, up to a log-linear approximation,

$$
\begin{aligned}
\check{m}_{t+1} & =-\mu_{x, t+1}\left[\check{\varpi}_{t+1}+\ell_{q, t+1}\left(\check{c}_{t+1, o}-\check{\varpi}_{t+1}\right)\right]-\mu_{q, t+1}\left(\check{c}_{t+1, o}-\check{\varpi}_{t+1}\right) \\
& =\left[\mu_{x, t+1}\left(\ell_{q, t+1}-1\right)+\mu_{q, t+1}\right] \check{\varpi}_{t+1}-\left(\mu_{x, t+1} \ell_{q, t+1}+\mu_{q, t+1}\right) \check{c}_{t+1, o} .
\end{aligned}
$$

Using the same calculation as for (99), one can then show that

$$
\begin{aligned}
\mathbf{S R}_{t}= & -\left[\mu_{x, t+1}\left(\ell_{q, t+1}-1\right)+\mu_{q, t+1}\right] \rho_{\mathrm{w}, R, t+1} \sigma_{\varpi, t+1} \\
& +\left(\mu_{x, t+1} \ell_{q, t+1}+\mu_{q, t+1}\right) \rho_{c, 0, R, t+1} \sigma_{c, 0, t+1} .
\end{aligned}
$$

For the specific funcational form of subsection 3.3, we thus obtain

$$
\mathbf{S R}_{t}=\frac{\eta-v}{1-\eta} \rho_{\varpi, R, t+1} \sigma_{\varpi, t+1}+v \rho_{c,, R, t+1} \sigma_{c, 0, t+1} .
$$


These equations are informative about measuring curvature parameters of the utility specification. In particular, we see that if there is no surprise longevity risk, $\check{\varpi}_{t+1} \equiv 0$, then the risk premium is proportional to the risk-aversion parameter $v$, the standard deviation $\sigma_{c, o, t+1}$ of old-age consumption $c_{o, t+1}$, and the correlation $\rho_{c, 0, R, t+1}$ of old-age consumption with the asset return.

In solving a social planner's problem and in characterizing the speed of capital accumulation, the appropriate risk premium and thus, the appropriate scarcity of capital to generate the required average return ought to be taken into account.

\section{A.4 Solving the Social Planner's Problem}

\section{A.4.1 Collecting the Equations}

For reference, the social planner's problem solves the following set of equations:

$$
\begin{aligned}
y_{t}= & A_{t} k_{t-1} f\left(Z_{t} n_{t}\right), \\
c_{t, y} \pi_{t}+c_{t, o}+k_{t} \pi_{t}= & {\left[A_{t} f\left(Z_{t} n_{t}\right)+1-\delta_{t}\right] k_{t-1^{\prime}} } \\
w_{t}= & A_{t} k_{t-1} f^{\prime}\left(Z_{t} n_{t}\right) \frac{Z_{t}}{\pi_{t}} \\
\frac{\partial u\left(c_{t, y}, n_{t}\right)}{\partial c_{t, y}} w_{t}= & -\frac{\partial u\left(c_{t, y^{\prime}} n_{t}\right)}{\partial n_{t}}, \\
R_{t}= & A_{t} f\left(Z_{t} n_{t}\right)+1-\delta_{t}, \\
\frac{\partial u\left(c_{t, y^{\prime}} n_{t}\right)}{\partial c_{t, y}}= & \beta\left(x^{-1}\right)^{\prime}\left(E_{t}\left\{x\left[\varpi_{t+1} q\left(\frac{c_{t+1, o}}{\varpi_{t+1}}\right)\right]\right\}\right) \\
z^{\prime}\left(a_{t}\right) \frac{\partial u\left(c_{t, y} n_{t}\right)}{\partial c_{t, y}}= & \frac{\beta}{\omega} z^{\prime}\left(a_{t-1}\right)\left(x^{\prime}\left[\varpi_{t+1} q\left(\frac{c_{t+1, o}}{\varpi_{t+1}}\right)\right] q^{\prime}\left(\frac{c_{t+1, o}}{\varpi_{t+1}}\right) R_{t+1}\right\} \\
& \cdot x_{t-1}^{\prime}\left\{x\left[\varpi_{t} q\left(\frac{c_{t, o}}{\varpi_{t}}\right)\right] q^{\prime}\left(\frac{c_{t, o}}{\varpi_{t}}\right)\right] \\
a_{t}= & u\left(c_{t, y}, n_{t}\right)+\beta x^{-1}\left(E_{t}\left\{x\left[\varpi_{t+1} q\left(\frac{c_{t+1, o}}{\varpi_{t+1}}\right)\right]\right\}\right) .
\end{aligned}
$$


We assume that preferences are given by (44). Define

$s=\frac{\eta}{1-\eta}(1-v)$

and note that

$\frac{1-\eta}{1-v} \varsigma=\eta$

which turns out to be useful in some calculations below. Note that

$$
\begin{gathered}
x\left[\boldsymbol{\varpi}_{t} q\left(\frac{c_{t, o}}{\boldsymbol{\varpi}_{t}}\right)\right]=\frac{1}{1-v} \boldsymbol{\varpi}_{t}^{5} c_{t, o}^{1-v} \\
x^{\prime}\left[\boldsymbol{\varpi}_{t} q\left(\frac{c_{t, o}}{\boldsymbol{\varpi}_{t}}\right)\right] q^{\prime}\left(\frac{c_{t, o}}{\boldsymbol{\varpi}_{t}}\right)=\boldsymbol{\varpi}_{t}^{5} c_{t, o}^{-v} .
\end{gathered}
$$

Thus the equations above become

$$
\begin{aligned}
y_{t} & =A_{t} k_{t-1} f\left(Z_{t} n_{t}\right), \\
c_{t, y} \pi_{t}+c_{t, o}+k_{t} \pi_{t} & =\left[A_{t} f\left(Z_{t} n_{t}\right)+1-\delta_{t}\right] k_{t-1}, \\
w_{t} & =A_{t} k_{t-1} f^{\prime}\left(Z_{t} n_{t}\right) \frac{Z_{t}}{\pi_{t}}, \\
\frac{w_{t}}{c_{t, y}} & =-\frac{v^{\prime}\left(n_{t}\right)}{v\left(n_{t}\right)}, \\
R_{t} & =A_{t} f\left(Z_{t} n_{t}\right)+1-\delta_{t \prime} \\
\frac{\beta}{\varpi} s_{t-1}^{-1} \varpi_{t}^{s} c_{t, o}^{-\nu} & =\left[(1-\eta) a_{t}\right]^{(\eta-\xi) /(1-\eta)} c_{t, y}^{-\eta} v\left(n_{t}\right)^{1-\eta}, \\
(1-\eta) a_{t} & =\left[c_{t, y} v\left(n_{t}\right)\right]^{1-\eta}+\beta x_{t}^{1-\eta}, \\
x_{t} & =\left[E_{t}\left(\varpi_{t+1}^{s} c_{t+1, o}^{1-\eta}\right)\right]^{1 /(1-v)}, \\
s_{t} & =\left[(1-\eta) a_{t}\right]^{(\xi-\eta) /(1-\eta)} x_{t}^{\eta-\nu},
\end{aligned}
$$

where the last two lines define $x_{t}$ and $s_{t}$.

\section{A.4.2 Normalization}

To turn this into a stationary system, the growing variables need to be divided by the beginning-of-period capital level $k_{t-1}$. Thus, let 


$$
\begin{aligned}
& \tilde{c}_{t, y} \equiv \frac{c_{t, y}}{k_{t-1}}, \\
& \tilde{c}_{t, o} \equiv \frac{c_{t, 0}}{k_{t-1}}, \\
& \tilde{w}_{t} \equiv \frac{w_{t}}{k_{t-1}}, \\
& \tilde{y}_{t} \equiv \frac{y_{t}}{k_{t-1}}, \\
& \tilde{k}_{t} \equiv \frac{k_{t}}{k_{t-1}}, \\
& \tilde{x}_{t} \equiv \frac{x_{t}}{k_{t-1}},
\end{aligned}
$$

except that we define

$$
\begin{aligned}
& \tilde{a}_{t} \equiv \frac{a_{t}}{k_{t-1}^{1-\eta}}, \\
& \tilde{s}_{t} \equiv s_{t} k_{t}^{\nu-\xi} .
\end{aligned}
$$

The exponent differs here in order to achieve the appropriate scaling for these utility variables. Moreover, we scale $s_{t}$ by $k_{t}^{\nu-\xi}$ rather than by $k_{t-1}^{v-\xi}$ because that way, $\tilde{s}_{t-1}$ (rather than $\tilde{s}_{t-1}$ and $\tilde{k}_{t-1}$ ) turns out to be the only remaining endogenous-state variable.

Rewrite the system as

$$
\begin{aligned}
\tilde{y}_{t} & =A_{t} f\left(Z_{t} n_{t}\right), \\
\tilde{c}_{t, y} \pi_{t}+\tilde{c}_{t, o}+\tilde{k}_{t} \pi_{t} & =A_{t} f\left(Z_{t} n_{t}\right)+1-\delta_{t}, \\
\tilde{w}_{t} & =A_{t} f^{\prime}\left(Z_{t} n_{t}\right) \frac{Z_{t}}{\pi_{t},} \\
\frac{\tilde{w}_{t}}{\tilde{c}_{t, y}} & =-\frac{v^{\prime}\left(n_{t}\right)}{v(n)}, \\
R_{t} & =A_{t} f\left(Z_{t} n_{t}\right)+1-\delta_{t}, \\
\tilde{c}_{t, y}^{-\eta} v\left(n_{t}\right)^{1-\eta} & =\beta \tilde{x}_{t}^{v-\eta} \tilde{k}_{t}^{-v} E_{t}\left(\varpi_{t+1}^{\mathrm{s}} \tilde{c}_{t+1, o}^{-v} R_{t+1}\right),
\end{aligned}
$$




$$
\begin{aligned}
\frac{\beta}{\varpi} \tilde{s}_{t-1}^{-1} \varpi \tilde{c}_{t, o}^{-v} & =\left[(1-\eta) \tilde{a}_{t}\right]^{(\eta-\xi) /(1-\eta)} \tilde{c}_{t, y}^{-\eta} v\left(n_{t}\right)^{1-\eta}, \\
(1-\eta) \tilde{a}_{t} & =\left[\tilde{c}_{t, y} v\left(n_{t}\right)\right]^{1-\eta}+\beta \tilde{x}_{t}^{1-\eta}, \\
\tilde{x}_{t} & =\tilde{k}_{t}\left[E_{t}\left(\varpi_{t+1}^{\varsigma} \tilde{c}_{t+1, o}^{1-v}\right)\right]^{1 /(1-v)}, \\
\tilde{s}_{t} & =\left[(1-\eta) \tilde{a}_{t}\right]^{(\xi-\eta) /(1-\eta)} \tilde{x}_{t}^{\eta-v} \tilde{k}_{t}^{v-\xi}
\end{aligned}
$$

Note that indeed the only endogenous state variable remaining is $\tilde{s}_{t-1}$.

\section{A.4.3 Stochastic Assumptions}

To make further progress, we need some additional, tractable assumptions. Let

$$
\begin{array}{r}
\varpi_{t} \\
A_{t} \\
\zeta_{t}=Z_{t} \\
\pi_{t} \\
\delta_{t}
\end{array}
$$

be the vector of exogenous parameters. We assume that a steady state $\bar{\zeta}$ exists and that

$\hat{\zeta}_{t}=\log \left(\zeta_{t}\right)-\log (\bar{\zeta})$

follows an $\mathrm{AR}(1)$ process,

$\hat{\zeta}_{t}=N \hat{\zeta}_{t-1}+\epsilon_{t}$

for some $5 \times 5$ matrix $N$ with nonexplosive roots, where

$$
\epsilon_{t}=\left(\begin{array}{c}
\epsilon_{\tilde{w}, t} \\
\epsilon_{A, t} \\
\epsilon_{Z, t} \\
\epsilon_{\pi, t} \\
\epsilon_{\delta, t}
\end{array}\right)
$$

is the vector of innovations for each exogenous parameter and $\epsilon_{t} \sim \mathcal{N}(0, \Sigma)$. 


\section{A.4.4 Steady State}

The steady state for the tilde variables-which we shall now also use as our benchmark values-is given by the equations

$$
\begin{aligned}
& \bar{y}=\bar{A} f(\bar{Z} \bar{n}), \\
& \bar{c}_{y} \bar{\pi}+\bar{c}_{o}+\bar{k} \bar{\pi}=\bar{A} f(\bar{Z} \bar{n})+1-\bar{\delta}, \\
& \bar{w}=\bar{A} f^{\prime}(\bar{Z} \bar{n}) \frac{\bar{Z}}{\bar{\pi}}, \\
& \frac{\bar{w}}{\bar{c}_{y}}=-\frac{v^{\prime}(\bar{n})}{v(\bar{n})}, \\
& \bar{R}=\bar{A} f(\bar{Z} \bar{n})+1-\bar{\delta}, \\
& \bar{c}_{y}^{-\eta} v(\bar{n})^{1-\eta}=\beta \bar{x}^{\nu-\eta} \bar{k}^{-\nu} \overline{\bar{\omega}}^{\varsigma} \bar{c}_{o}^{-\nu} \bar{R} \exp \left(\frac{\sigma_{R-\nu c}^{2}}{2}\right) \text {, } \\
& \frac{\beta}{\omega} \bar{s}^{-1} \overline{\boldsymbol{\omega}}^{s} \bar{c}_{o}^{-\nu}=[(1-\eta) \bar{a}]^{(\eta-\xi) /(1-\eta)} \bar{c}_{y}^{-\eta} v(\bar{n})^{1-\eta}, \\
& (1-\eta) \bar{a}=\left[\bar{c}_{y} v(\bar{n})\right]^{1-\eta}+\beta \bar{x}^{1-\eta}, \\
& \bar{x}=\bar{k}\left[\exp \left(\frac{\sigma_{x}^{2}}{2}\right) \bar{\omega}^{\varsigma} \bar{c}_{o}^{1-\nu}\right]^{1 /(1-v)}, \\
& \overline{\mathrm{s}}=[(1-\eta) \bar{a}]^{(\xi-\eta) /(1-\eta)} \bar{x}^{\eta-v} \bar{k}^{\nu-\xi},
\end{aligned}
$$

where $\sigma_{R-v c}^{2}$ denotes the conditional variance of $\log R_{t+1}+\varsigma \log \left(\varpi_{t+1}\right)-$ $v \log \left(c_{t+1,0}\right)$ and $\sigma_{x}^{2}$ is the conditional variance of $\mathrm{s} \log \varpi_{t+1}+(1-v) \log$ $c_{t+1, o}$.

We have included the risk terms in the intertemporal equations, thus including a precautionary motive for saving. We implicitly assumed (as remains to be shown in the log-linearized version), that the variances are constant over time. This will be justified below, when solving for the linear recursive law of motion for the loglinearized system (see subsection A.4.10).

Finally, define the argument share

$\alpha=\frac{\left[\bar{c}_{y} v(\bar{n})\right]^{1-\eta}}{(1-\eta) \bar{a}}$

for $\eta \neq 1$ and 


$$
\begin{aligned}
& \alpha=\frac{1}{1+\beta} \\
& \text { for } \eta=1
\end{aligned}
$$

\section{A.4.5 Solving for the Steady State}

Labor supply (108) can be written as

$$
\frac{\bar{w} \bar{n}}{\bar{c}_{y}}=-\ell_{v}(\bar{n}) \text {, }
$$

which says that the inverse of the share of wage income spent on consumption when young is tied to $\ell_{v}(\bar{n})$. Labor demand (107) together with (105) implies

$$
\frac{\bar{w} \bar{n} \bar{\pi}}{\bar{y}}=\ell_{f}(\bar{Z} \bar{n})
$$

The labor share in production is thus closely related to the productivity weighted labor input $\bar{Z} \bar{n}$. Combining equations (115) and (116), one can eliminate the wage rate $\bar{w}$

$$
\frac{\bar{\pi} \bar{c}_{y}}{\bar{y}}=\frac{\ell_{f}(\bar{Z} \bar{n})}{-\ell_{v}(\bar{n})} \text {. }
$$

We also assume that variances and covariances are known: the underlying fixed-point problem will be discussed below. Equation (113) implies $\bar{x}=\kappa_{1} \bar{k} \bar{c}_{o}$,

where

$\kappa_{1}=\exp \left(\frac{\sigma_{x}^{2} / 2}{1-v}\right) \overline{\boldsymbol{\omega}}^{\varsigma /(1-v)}$.

The asset pricing equation (110), which can be viewed as describing optimal saving, can be rewritten with (118) as

$\bar{c}_{y}^{-\eta} v(\bar{n})^{1-\eta}=\kappa_{2} \bar{c}_{0}^{-\eta} \bar{k}^{-\eta} \bar{R}$,

where

$$
\begin{aligned}
& \kappa_{2}=\beta \kappa_{1}^{\nu-\eta} \varpi^{\zeta} \exp \left(\frac{\sigma_{R-v c}^{2}}{2}\right) \\
& =\beta \bar{\varpi}^{\eta} \exp \left(\frac{\nu-\eta}{1-\nu} \frac{\sigma_{x}^{2}}{2}\right) \exp \left(\frac{\sigma_{R-v c}^{2}}{2}\right) .
\end{aligned}
$$


The risk-sharing condition (111) together with the definitions (114) and (118) implies

$$
\vec{c}_{y}^{-\eta} \mathcal{V}(\bar{n})^{1-\eta}=\left(\frac{\beta}{\omega} \kappa_{1}^{\nu-\eta} \overline{\sigma^{\sigma}} \zeta\right) \bar{c}_{o}^{-\eta} \bar{k}^{\xi-\eta},
$$

which can be rewritten as

$$
\frac{\bar{c}_{o}}{\bar{\pi} \bar{c}_{y}}=\kappa_{3} \bar{k}^{(\xi-\eta) / \eta} v(\bar{n})^{(\eta-1) / \eta},
$$

where

$$
\begin{aligned}
\kappa_{3} & =\frac{1}{\bar{\pi}}\left(\frac{\beta}{\omega} \kappa_{1}^{\nu-\eta} \overline{\sigma^{\zeta}}\right)^{1 / \eta} \\
& =\frac{1}{\bar{\pi}}\left(\frac{\beta}{\omega}\right)^{1 / \eta} \bar{\varpi} \exp \left[\frac{\nu-\eta}{\eta(1-\nu)} \frac{\sigma_{x}^{2}}{2}\right] .
\end{aligned}
$$

While (119) arises from an intertemporal savings decision, equation (120) arises from the risk-sharing condition. The difference between these two equations thus partially stems from the different weights given to an agent currently alive or alive in the future due to population growth and social planner discounting. Equations (119) and (120) with (109) together imply

$$
\bar{k}^{\xi}=\kappa_{4} \bar{R}=\kappa_{4}[\bar{A} f(\bar{Z} \bar{n})+1-\bar{\delta}] \text {, }
$$

where

$$
\kappa_{4}=\frac{\omega \kappa_{2}}{\beta \kappa_{1}^{\nu-\eta} \varpi^{\zeta}}=\omega \exp \left(\frac{\sigma_{R-v c}^{2}}{2}\right),
$$

which sheds light on the relationship between growth of the economy $\bar{k}$ and required labor $\bar{n}$ versus the discount factor of the social planner $\omega$ and a term $\exp \left(\sigma_{R-v c}^{2}\right)$ related to the risk premium. Note also that

$$
\kappa_{3}=\frac{1}{\bar{\pi}}\left(\frac{\kappa_{2}}{\kappa_{4}}\right)^{1 / \eta} \text {. }
$$

The right-hand side of (106) equals $\bar{R}$. Use (105) to rewrite (106) as

$$
\frac{\bar{\pi} \bar{c}_{y}}{\bar{y}}\left(1+\frac{\bar{c}_{o}}{\bar{\pi} \bar{c}_{y}}\right)=1+\frac{1-\bar{\delta}-\bar{\pi} \bar{k}}{\bar{A} f(\bar{Z} \bar{n})} .
$$

Combining equations in $\bar{\pi} \bar{c}_{y} / \bar{y}, \bar{c}_{\mathrm{o}} / \bar{\pi} \bar{c}_{y^{\prime}}, \bar{k}$, and $\bar{n}$, we have 


$$
\begin{aligned}
\frac{\bar{\pi} \bar{c}_{y}}{\bar{y}}\left(1+\frac{\bar{c}_{o}}{\bar{\pi} \bar{c}_{y}}\right) & =1+\frac{1-\bar{\delta}-\bar{\pi} \bar{k}}{\bar{A} f(\bar{Z} \bar{n})}, \\
\frac{\bar{\pi} \bar{c}_{y}}{\bar{y}} & =\frac{\ell_{f}(\bar{Z} \bar{n})}{-\ell_{v}(\bar{n})}, \\
\frac{\bar{c}_{o}}{\bar{\pi} \bar{c}_{y}} & =\kappa_{3} \bar{k}^{(\xi-\eta \eta \eta) / \eta} v(\bar{n})^{(\eta-1) / \eta}, \\
\bar{k}^{\xi} & =\kappa_{4} \bar{R}=\kappa_{4}[\bar{A} f(\bar{Z} \bar{n})+1-\bar{\delta}] .
\end{aligned}
$$

Substituting the second and third equation into the first to eliminate $\bar{\pi} \bar{c}_{y} / \bar{y}$ and $\bar{c}_{o} / \bar{\pi} \bar{c}_{y}$, one obtains

$\left[\frac{\ell_{f}(\bar{Z} \bar{n})}{-\ell_{v}(\bar{n})}\right]\left[1+\kappa_{3} \bar{k}^{(\xi-\eta) / \eta} v(\bar{n})^{(\eta-1) / \eta}\right]=1+\frac{1-\bar{\delta}-\bar{\pi} \bar{k}}{\bar{A} f(\bar{Z} \bar{n})}$.

Finally, use the fourth equation (127) to eliminate $\bar{k}$ to obtain

$$
\begin{aligned}
& {\left[\frac{\ell_{f}(\bar{Z} \bar{n})}{-\ell_{v}(n)}\right]\left(1+\kappa_{3}\left\{\kappa_{4}[\bar{A} f(\bar{Z} \bar{n})+1-\bar{\delta}]\right\}^{(\xi-\eta) / \bar{\xi} \eta} v(\bar{n})^{(\eta-1) / \eta}\right)} \\
& =1+\frac{1-\bar{\delta}-\bar{\pi}\left\{\kappa_{4}[\overline{A f}(\bar{Z} \bar{n})+1-\bar{\delta}]\right\}^{1 / \xi}}{\bar{A} f(\bar{Z} \bar{n})} .
\end{aligned}
$$

This is a single and nonlinear equation in $\bar{n}$. Solving it requires a specification for $v(\cdot)$ and $f(\cdot)$. A solution may perhaps be given in special cases, but numerical methods must be used generally. There may be multiple solutions $\bar{n}>0$, indicating a multiplicity of steady states. Given a solution to this equation, all other steady state variables can then be calculated.

\section{A.4.6 A Parameterization}

We shall assume that

$$
\begin{aligned}
& v(n)=\bar{v} \frac{(1-n)^{1-\chi}}{1-\chi} \\
& \text { with } 0 \leq \chi<1, \text { so that } \\
& \ell_{v}(n)=-(1-\chi) \frac{n}{1-n}, \\
& \mu_{v}(n)=-\chi \frac{n}{1-n} .
\end{aligned}
$$


Thus, (115) can be rewritten as

$$
\frac{\bar{w}(1-\bar{n})}{\bar{c}_{y}}=1-\chi .
$$

The expenditure ratio of leisure over consumption when young is thus given by $1-\chi$.

Further, we assume that

$f(x)=\left[\theta+(1-\theta) x^{1-(1 / \psi)}\right]^{1 /[1-(1 / \psi)]}$

with $0 \leq \theta<1$ and $\psi>0$ (where one should note that we usually use $x=$ $\mathrm{Zn}$ as argument), and thus

$\ell_{f}(x)=\frac{(1-\theta) x^{1-(1 / \psi)}}{\theta+(1-\theta) x^{1-(1 / \psi)^{\prime}}}$

$\mu_{f}(x)=\frac{1}{\psi}\left[1-\ell_{f}(x)\right]$.

For $\psi \rightarrow 1$, this becomes

$f(x)=x^{1-\theta}$

Proof. Let $\varepsilon=1-(1 / \psi)$. Note that

$$
\begin{aligned}
\log f(x ; \varepsilon) & =\frac{1}{\varepsilon} \log \{1+(1-\theta)[\exp (\varepsilon \log x)-1]\} \\
& \approx \frac{1}{\varepsilon}(1-\theta)[\exp (\varepsilon \log x)-1] \\
& \approx \frac{1}{\varepsilon}(1-\theta)(\varepsilon \log x) \\
& =(1-\theta) \log x,
\end{aligned}
$$

which delivers the claim.

Either directly or per $\psi \rightarrow 1$, one then gets

$$
\ell_{f}(x)=1-\theta,
$$

$\mu_{f}(x)=\theta$. 


\section{A.4.7 A Special Case}

We now analyze the case $\psi=1, \xi=\eta \rightarrow 1, \bar{\delta}=1$ per successively investigating the implications of each additional restriction.

For the special case $\psi=1$, replace in equation (125) to get

$$
\frac{\bar{\pi} \bar{c}_{y}}{\bar{y}}=\left(\frac{1}{\bar{n}}-1\right) \frac{(1-\theta)}{(1-\chi)^{\prime}}
$$

and in equation (128) to find

$$
\begin{aligned}
& \left(\frac{1}{\bar{n}}-1\right) \frac{(1-\theta)}{(1-\chi)} \\
& \cdot\left(1+\kappa_{3}\left\{\kappa_{4}\left[\bar{A}(\bar{Z} \bar{n})^{1-\theta}+1-\bar{\delta}\right]\right\}^{(\xi-\eta) / \xi \eta}\left[\frac{\bar{v}(1-\bar{n})^{1-\chi}}{1-\chi}\right]^{(\eta-1) / \eta}\right) \\
& =1+\frac{1-\bar{\delta}-\bar{\pi}\left\{\kappa_{4}\left[\bar{A}(\bar{Z} \bar{n})^{1-\theta}+1-\bar{\delta}\right]\right\}^{1 / \chi}}{\bar{A}(\bar{Z} \bar{n})^{1-\theta}} .
\end{aligned}
$$

For $\xi=\eta \rightarrow 1$, the ratio of old-age consumption to young-age consumption is given by (126) as

$$
\frac{\bar{c}_{o}}{\bar{c}_{y}}=\bar{\pi} \kappa_{3}=\frac{\beta}{\omega} \exp \left(\frac{-\sigma_{x}^{2}}{2}\right) \text {. }
$$

Furthermore, for $\xi=\eta \rightarrow 1$, one obtains

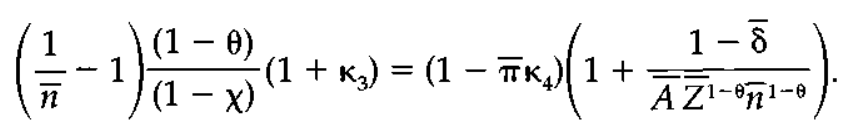

With complete depreciation (i.e., $\bar{\delta}=1$ ), this becomes

$\bar{n}=\left[1+\kappa_{5} \frac{(1-\chi)}{(1-\theta)}\right]^{-1}$,

where

$\frac{\bar{\pi} \bar{c}_{y}}{\bar{y}}=\kappa_{5}=\frac{1-\bar{\pi} \kappa_{4}}{1+\kappa_{3}}$.

A closed-form solution is thus available in this case. Ignoring the variance and risk premium terms or assuming a nonstochastic steady state, we can write $\kappa_{5}$ as

$\frac{\bar{\pi} \bar{c}_{y}}{\bar{y}}=\kappa_{5}=\frac{1-\bar{\pi} \omega}{1+\beta(\bar{\pi} \omega)^{-1}}$ 
so that (by substitution into [136]),

$\bar{n}=\left\{1+\frac{(1-\bar{\pi} \omega)}{\left[1+\beta(\bar{\pi} \omega)^{-1}\right]} \frac{(1-\chi)}{(1-\theta)}\right\}^{-1}$,

where one can now investigate the impact of a variety of parameters on the steady state.

A particularly simple case is $\beta=\omega$ and $\bar{\pi}=1$. Ignoring the variance and risk premium terms, one has

$$
\begin{aligned}
& \frac{\bar{c}_{y}}{\bar{y}}=\frac{\bar{c}_{o}}{\bar{y}}=\frac{1-\beta}{2} \\
& \bar{n}=\left[1+\frac{(1-\beta)}{2} \frac{(1-\chi)}{(1-\theta)}\right]^{-1}
\end{aligned}
$$

with the gross-investment-to-GDP ratio $(\bar{\pi} k) / \bar{y}$ given by $\beta$.

Given a value of $\bar{n}$, equation (127) shows that the growth factor $\bar{k}$ of the economy and the level of total factor productivity $\bar{A}$ are closely related. Varying the latter will not affect $\bar{n}$ under full depreciation in case $\xi=\eta$ $\rightarrow 1$, as shown above. Thus, to obtain an economy with a growth factor $\vec{k}$, we can set

$\bar{A}=\frac{\bar{k}}{\omega(\bar{Z} \bar{n})^{\theta}}$,

where we once again ignore the variance and risk premium terms.

These calculations also help in a somewhat more general case. For suppose, alternatively, that $\bar{\delta}=1$, but that $\xi \neq \eta \neq 1$. Use the solution $\bar{n}$ of equation (135), but without imposing $\eta=1$ for calculating $\kappa_{5}$, that is, using (136) to back out the level parameter $\bar{v}$ in the disutility of the young consistent with this steady state

$\bar{v}=\left\{\kappa_{4}\left[\bar{A}(\bar{Z} \bar{n})^{1-\theta}+1-\bar{\delta}\right]\right\}^{(\xi-\eta) /[\xi(1-\eta)]}\left[\frac{(1-\bar{n})^{1-\chi}}{1-\chi}\right]^{-1}$.

This may be useful in order to investigate local comparative statics around a known steady state or to find an initial point for numerically calculating the steady state.

\section{A.4.8 Loglinearization}

We now use hats on variables to denote the loglinearization of the detrended variables around the detrended steady state, for example, $\hat{c}_{t, y}=\log \left(\tilde{c}_{t, y}\right)-\log \left(\bar{c}_{t, y}\right)$. 
Furthermore, and from here onward and in slight abuse of notation, let $\ell_{f}$ and $\ell_{v}$ be the logarithmic derivatives of $f(\cdot)$ and $v(\cdot)$, and $\mu_{f}$ and $\mu_{v}$, the (negative) elasticity of $f^{\prime}(\cdot)$ and $v^{\prime}(\cdot)$, all evaluated at steady-state employment $\bar{n}$ for $v(\cdot)$, respectively $\bar{Z} \bar{n}$ for $f(\cdot)$. The loglinearization is given by

$$
\begin{aligned}
\hat{y}_{t}= & \hat{A}_{t}+\ell_{f}\left(\hat{Z}_{t}+\hat{n}_{t}\right), \\
& \overline{c_{y}} \bar{\pi}\left(\hat{c}_{t, y}+\hat{\pi}_{t}\right)+\bar{c}_{o} \hat{c}_{t, o}+\bar{k} \bar{\pi}\left(\hat{k}_{t}+\hat{\pi}_{t}\right) \\
= & \bar{y}\left[\hat{A}_{t}+\ell_{f}\left(\hat{Z}_{t}+\hat{n}_{t}\right)\right]-\bar{\delta} \hat{\delta}_{t \prime} \\
\hat{w}_{t}= & \hat{A}_{t}-\mu_{f}\left(\hat{Z}_{t}+\hat{n}_{t}\right)+\hat{Z}_{t}-\hat{\pi}_{t \prime} \\
\hat{w}_{t}-\hat{c}_{t, y}= & -\left(\mu_{v}+\ell_{v}\right) \hat{n}_{t \prime} \\
\bar{R} \hat{R}_{t}= & \bar{y}\left[\hat{A}_{t}+\ell_{f}\left(\hat{Z}_{t}+\hat{n}_{t}\right)\right]-\bar{\delta} \hat{\delta}_{t \prime} \\
-\eta \hat{c}_{t, y}+(1-\eta) \hat{\ell}_{v} \hat{n}_{t}= & -\eta \hat{k}_{t}+E_{t}\left(\eta \hat{\varpi}_{t+1}-\eta \hat{c}_{t+1, o}+\hat{R}_{t+1}\right), \\
\hat{s}_{t-1}+v \hat{c}_{t, o}-s \hat{\varpi}_{t}= & (\xi-\eta)\left(\frac{\hat{a}_{t}}{1-\eta}\right)+\eta \hat{c}_{t, y}-(1-\eta) \ell_{v} \hat{n}_{t \prime} \\
\left(\frac{\hat{a}_{t}}{1-\eta}\right)= & \alpha\left(\hat{c}_{t, y}+\ell_{v} \hat{n}_{t}\right) \\
& +(1-\alpha)\left[\hat{k}_{t}+E_{t}\left(\frac{\eta}{1-\eta} \hat{\varpi}_{t+1}+\hat{c}_{t, 1, o}\right)\right], \\
\hat{s}_{t}= & (\xi-\eta)\left[\left(\frac{\hat{a}_{t}}{1-\eta}\right)-\hat{k}_{t}\right] \\
& +(\eta-v) E_{t}\left(\frac{\eta}{1-\eta} \hat{\varpi}_{t+1}+\hat{c}_{t+1, o}\right),
\end{aligned}
$$

where we replaced $\hat{x}_{t}$ everywhere with

$\hat{x}_{t}=\hat{k}_{t}+E_{t}\left(\frac{\eta}{1-\eta} \hat{\varpi}_{t+1}+\hat{c}_{t+1, o}\right)$.

\section{A.4.9 Preparation for MATLAB Implementation}

In order to implement this system of equations, it is more convenient to use $\hat{\delta}_{t}^{*}=\bar{\delta} \hat{\delta}_{t}$ - since this is already in percent anyways and to handle $\bar{\delta}=$ 0 as limit - as well as $\hat{a}_{t}^{*}=\left[\left(\hat{a}_{t} /(1-\eta)\right]\right.$. Write the system as 


$$
\begin{aligned}
& 0=\hat{A}_{t}+\ell_{f}\left(\hat{Z}_{t}+\hat{n}_{t}\right)-\hat{y}_{t \prime} \\
& 0=\bar{R} \hat{R}_{t}-\bar{c}_{y} \bar{\pi}\left(\hat{c}_{t, y}+\hat{\pi}_{t}\right)-\bar{c}_{\mathrm{o}} \hat{c}_{t, o}-\bar{k} \bar{\pi}\left(\hat{k}_{t}+\hat{\pi}_{t}\right), \\
& 0=\hat{A}_{t}-\mu_{f}\left(\hat{Z}_{t}+\hat{n}_{t}\right)+\hat{Z}_{t}-\hat{\pi}_{t}-\hat{w}_{t \prime} \\
& 0=-\left(\mu_{v}+\ell_{v}\right) \hat{n}_{t}-\hat{w}_{t}+\hat{c}_{t, y} \\
& 0=\bar{y} \hat{y}_{t}-\hat{\delta}_{t}^{*}-\bar{R} \hat{R}_{t \prime} \\
& \left.0=(\xi-\eta) \hat{a}_{t}^{*}+\eta \hat{c}_{t, y}-(1-\eta) \ell_{v} \hat{n}_{t}-\hat{s}_{t-1}-v \hat{c}_{t, o}+s \hat{\pi}_{t}\right), \\
& 0=-\eta \hat{k}_{t}+E_{t}\left(\eta \hat{\varpi}_{t+1}-\eta \hat{c}_{t+1, o}+\hat{R}_{t+1}\right)+\eta \hat{c}_{t, y}-(1-\eta) \ell_{v} \hat{n}_{t \prime} \\
& 0=\alpha\left(\hat{c}_{t, y}+\ell_{v} \hat{n}_{t}\right)-\hat{a}_{t}^{*}+(1-\alpha)\left[\hat{k}_{t}+E_{t}\left(\frac{\eta}{1-\eta} \hat{\boldsymbol{\sigma}}_{t+1}+\hat{c}_{t+1, o}\right)\right] \\
& 0=(\xi-\eta)\left(\hat{a}_{t}^{*}-\hat{k}_{t}\right)-\hat{s}_{t}+(\eta-v) E_{t}\left(\frac{\eta}{1-\eta} \hat{\varpi}_{t+1}+\hat{c}_{t+1, o}\right) .
\end{aligned}
$$

In order to incorporate the generational account perspective, we also add the equations

$$
\begin{aligned}
& 0=\hat{\tau}_{t, \text { priv }}+\frac{\bar{c}_{y}}{\bar{w} \bar{n}}\left(\hat{c}_{y, t}-\hat{w}_{t}-\hat{n}_{t}\right)+\frac{\bar{k}}{\bar{w} \bar{n}}\left(\hat{k}-\hat{w}_{t}-\hat{n}_{t}\right) \\
& 0=\hat{\tau}_{t, \text { pub }}+\frac{\bar{c}_{y}}{\bar{w} \bar{n}}\left(\hat{c}_{y, t}-\hat{w}_{t}-\hat{n}_{t}\right)
\end{aligned}
$$

to capture the movements in the ratios of lump-sum taxes to wage earnings to be paid by the young, expressed in percentage (rather than in percentage deviation from the steady state of this ratio).

As state variables we choose $\hat{s}_{t}, \hat{c}_{t, y}, \hat{c}_{t, o}, \hat{a}_{t}^{*}$, and $k$. The first three come in due to the insights from some analytic calculations toward deriving the law of motion in closed form, available as a technical appendix in a working paper version, and are to have three rather than one equation containing expectations. The variable $\hat{a}_{t}^{*}$ has been added in order to avoid potential difficulties of a purely algebraic nature in the special case $\xi=\eta$. Finally, $k$ has been added as a state to recalculate the impulse responses for the non-normalized variables, if so desired.

\section{A.4.10 The Recursive Law of Motion}

We wish to solve for the linear recursive law of motion, 


$$
\begin{aligned}
& \hat{s}_{t}=\varphi_{s, s} \hat{s}_{t-1}+\varphi_{s, \zeta} \zeta_{t} \\
& \hat{n}_{t}=\varphi_{n, s} \hat{s}_{t-1}+\varphi_{n, \zeta} \zeta_{t} \\
& \hat{c}_{t, y}=\varphi_{c y, s} \hat{s}_{t-1}+\varphi_{c y, \zeta} \zeta_{t} \\
& \hat{c}_{t, o}=\varphi_{c o, s} \hat{s}_{t-1}+\varphi_{c o, \zeta} \zeta_{t} \\
& \hat{R}_{t}=\varphi_{R, s} \hat{s}_{t-1}+\varphi_{R, \zeta} \zeta_{t}
\end{aligned}
$$

and so forth: that is, we wish to solve for the coefficients $\varphi_{(\cdot, s)} \in \mathfrak{M}$ and $\varphi_{(\cdot, 5)}$ $\in \mathfrak{R}^{5}$ such that the linear recursive law of motion satisfies the loglinearized equations. Note that the linear recursive law of motion implies that the conditional variances and covariances are constant and given by, for example,

$$
\begin{aligned}
\sigma_{c, o}^{2} & =\varphi_{c o, \zeta} \Sigma \varphi_{c o, \zeta}^{\prime} \\
\rho_{c, o, R} \sigma_{c, o} \sigma_{R} & =\varphi_{c o, \zeta} \Sigma \varphi_{R, \zeta}^{\prime} .
\end{aligned}
$$

In particular, the Sharpe ratio (100) can now be calculated.

In principle, this involves the calculation of a fixed point: the steady state requires knowledge of these variances and covariances, which can be calculated, given the linear recursive law of motion. But the latter is a solution to system of equations whose coefficients depend on the steady state. An iterative procedure typically works well. As a first step, assume these variances, covariances, and the Sharpe ratio to be zero, in which case one obtains the nonstochastic steady state. Use it to generate the loglinear approximation and solve it for the recursive law of motion. Calculate the implied variances and covariances, and use them to recalculate the steady state, and so on.

This procedure was used, for example, Canton $(1997,2002)$ in a different context. The procedure typically converges fast. In fact, a single step typically often suffices for all practical purposes. For that, use the nonstochastic steady state to generate a linear recursive law of motion and use the latter to calculate variances and covariances.

\section{A.5 A Closed-Form Solution}

We shall now provide a closed-form solution for the recursive law of motion, given the loglinearized system. The procedure follows the methodology explained in Uhlig (1999). We proceed in three steps. The first two steps concentrate entirely on calculating the deterministic law of motion. In the first step, we reduce the loglinearized equations to a 
system of three equations in $\hat{s}_{t}, \hat{c}_{t, y}$, and $\hat{c}_{t, o}$, and their leads and lags. Plugging in the recursive law of motion, we obtain a system of three equations in the three coefficients $\varphi_{c y, s}, \varphi_{c o, s}$, and $\varphi_{s, s}$, which we reduce to a quadratic equation in $\varphi_{s, s}$ and solve in the second step. With the solution to the deterministic part at hand, we proceed to calculate the coefficients on the stochastic part by solving a linear system of equations in the third step.

\section{A.5.1 Step 1: Reducing the System}

Due to linearity, the solution to the deterministic part is obtained by solving the loglinearized system under the assumption that all shocks are equal to zero, that is,

$$
\begin{aligned}
\hat{y}_{t} & =\ell_{f} \hat{n}_{t} \\
\bar{c}_{y} \bar{\pi} \hat{c}_{t, y}+\bar{c}_{o} \hat{c}_{t, o}+\bar{k} \bar{\pi} \hat{k}_{t} & =\bar{A} f(\bar{Z} \bar{n}) \ell_{f} \hat{n}_{t} \\
\hat{w}_{t} & =-\mu_{f} \hat{n}_{t} \\
\hat{w}_{t}-\hat{c}_{t, y} & =-\left(\mu_{v}+\ell_{v}\right) \hat{n}_{t} \\
\bar{R} \hat{R}_{t} & =\bar{A} f(\bar{Z} \bar{n}) \ell_{f} \hat{n}_{t} \\
-\eta \hat{c}_{t, y}+(1-\eta) \ell_{v} \hat{n}_{t} & =-\eta \hat{k}_{t}-\eta \hat{c}_{t+1, o}+\hat{R}_{t+1} \\
\hat{s}_{t-1}+\nu \hat{c}_{t, o} & =(\xi-\eta)\left(\frac{\hat{a}_{t}}{1-\eta}\right)+\eta \hat{c}_{t, y}-(1-\eta) \ell_{v} \hat{n}_{t} \\
\left(\frac{\hat{a}_{t}}{1-\eta}\right) & =\alpha\left(\hat{c}_{t, y}+\ell_{v} \hat{n}_{t}\right)+(1-\alpha)\left(\hat{k}_{t}+\hat{c}_{t+1, o}\right) \\
\hat{s}_{t} & =(\xi-\eta)\left[\left(\frac{\hat{a}_{t}}{1-\eta}\right)-\hat{k}_{t}\right]+(\eta-v) \hat{c}_{t+1, o}
\end{aligned}
$$

The first equation (160) is not needed for the reduction. Use the third and the fourth equation (162), (163) to express $\hat{n}_{t}$ in terms of $\hat{c}_{t, y}$. Use that in the second equation (161) to express $\hat{k}_{t}$ in terms of $\hat{c}_{t, y}$ and $\hat{c}_{t, o}$, in the fifth equation (164) to express $\hat{R}_{t}$ in terms of $\hat{c}_{t, y}$, and in the seventh equation (166) to express $\hat{a}_{t} /(1-\eta)$ in terms of $\hat{c}_{t, y^{\prime}} \hat{c}_{t, o}$ and $\hat{c}_{t+1, o^{\prime}}$

$$
\begin{aligned}
& \hat{n}_{t}=\theta_{n, y} \hat{c}_{t, y} \\
& \hat{k}_{t}=\theta_{k, y} \hat{c}_{t, y}+\theta_{k, o} \hat{c}_{t, o}
\end{aligned}
$$




$$
\begin{aligned}
\hat{R}_{t} & =\theta_{R, y} \hat{c}_{t, y} \\
\frac{\hat{a}_{t}}{1-\eta} & =\theta_{a, y} \hat{c}_{t, y}+\theta_{a, o} \hat{c}_{t, o}+\theta_{a, E v} \hat{c}_{t+1, o}
\end{aligned}
$$

where

$$
\begin{aligned}
& \theta_{n, y}=\frac{1}{\mu_{v}+\ell_{v}-\mu_{f}} \\
& \theta_{k, y}=\frac{\bar{A} f(\bar{Z} \bar{n}) \ell_{f}}{\bar{k} \bar{\pi}} \theta_{n, y}-\frac{\bar{c}_{y}}{\bar{k}} \\
& \theta_{k, o}=-\frac{\overline{c_{o}}}{\bar{k} \bar{\pi}} \\
& \theta_{R, y}=\frac{\bar{A} f(\bar{Z} \bar{n}) \ell_{f}}{\bar{R}} \theta_{n, y} \\
& \theta_{a, y}=\alpha\left(1+\ell_{v} \theta_{n, y}\right)+(1-\alpha) \theta_{k, y} \\
& \theta_{a, o}=(1-\alpha) \theta_{k, o} \\
& \theta_{a, E_{0}}=1-\alpha .
\end{aligned}
$$

Use these results in the remaining sixth, seventh, and ninth equation (165) (166) and (168) to obtain

$$
\begin{aligned}
0= & \theta_{1, y} \hat{c}_{t, y}+\theta_{1,0} \hat{c}_{t, o}+\theta_{1, E y} \hat{\mathcal{c}}_{t+1, y} & +\theta_{1, E 0} \hat{c}_{t+1, o} \\
s_{t-1}= & \theta_{2, y} \hat{c}_{t, y}+\theta_{2,0} \hat{c}_{t, o} & +\theta_{2, E o} \hat{c}_{t+1, o} \\
0=-s_{t}+\theta_{3, y} \hat{c}_{t, y}+\theta_{3,0} \hat{c}_{t, o} & & +\theta_{3, E 0} \hat{c}_{t+1, o}
\end{aligned}
$$

where

$$
\begin{aligned}
\theta_{1, y} & =\eta-(1-\eta) \ell_{v} \theta_{n, y}-\eta \theta_{k, y} \\
\theta_{1, o} & =-\eta \theta_{k, o} \\
\theta_{1, E y} & =\theta_{R, y} \\
\theta_{1, E o} & =-\eta \\
\theta_{2, y} & =(\xi-\eta) \theta_{a, y}+\eta-(1-\eta) \ell_{v} \theta_{n, y} \\
\theta_{2, o} & =(\xi-\eta) \theta_{a, o}-\nu \\
\theta_{2, E o} & =(\xi-\eta) \theta_{a, E o}
\end{aligned}
$$




$$
\begin{aligned}
\theta_{3, y} & =(\xi-\eta)\left(\theta_{a, y}-\theta_{k, y}\right) \\
\theta_{3, o} & =(\xi-\eta)\left(\theta_{a, o}-\theta_{k, o}\right) \\
\theta_{3, E o} & =(\xi-\eta) \theta_{a, E o}+\eta-v .
\end{aligned}
$$

\section{A.5.2 Step 2: A System of Three Coefficient Equations}

Use the deterministic part of the recursive law of motion

$$
\begin{gathered}
\hat{s}_{t}=\varphi_{s, s} \hat{s}_{t-1} \\
\hat{c}_{t, y}=\varphi_{c y, s} \hat{s}_{t-1} \\
\hat{c}_{t, o}=\varphi_{c o s s} \hat{s}_{t-1}
\end{gathered}
$$

to replace all variables except $s_{t-1}$. For variables dated $t+1$, this requires plugging in twice. Comparing coefficients (or, equivalently, dividing by $\left.s_{t-1}\right)$ yields

$$
\begin{array}{lll}
0= & \theta_{1, y} \varphi_{c y, s}+\theta_{1, o} \varphi_{c o, s}+\theta_{1, E y} \varphi_{s, s} \varphi_{c y, s} & +\theta_{1, E o} \varphi_{s, s} \varphi_{c o, s} \\
1= & \theta_{2, y} \varphi_{c y, s}+\theta_{2, o} \varphi_{c o, s} & +\theta_{2, E o} \varphi_{s, s} \varphi_{c o, s} \\
0=-\varphi_{s, s}+\theta_{3, y} \varphi_{c y, s}+\theta_{3, o} \varphi_{c o, s} & +\theta_{3, E o} \varphi_{s, s} \theta_{c o, s}
\end{array}
$$

Multiply the second equation with $\theta_{3, E o}$ and the third with $-\theta_{2, E o}$, add and solve for $\varphi_{c y, s}$,

$$
\varphi_{c y, s}=\theta_{c y}+\theta_{c y, s} \varphi_{s, s}+\theta_{c y, c o} \varphi_{c o, s}
$$

where

$$
\begin{gathered}
\theta_{c y}=\frac{\theta_{3, E o}}{\boldsymbol{\theta}_{3, E o} \boldsymbol{\theta}_{2, y}-\boldsymbol{\theta}_{2, E_{o}} \boldsymbol{\theta}_{3, y}} \\
\boldsymbol{\theta}_{c y, s}=\frac{-\boldsymbol{\theta}_{2, E o}}{\boldsymbol{\theta}_{3, E o} \boldsymbol{\theta}_{2, y}-\boldsymbol{\theta}_{2, E_{0}} \boldsymbol{\theta}_{3, y}} \\
\boldsymbol{\theta}_{c y, c o}=\frac{\boldsymbol{\theta}_{2, E o} \boldsymbol{\theta}_{3, o}-\boldsymbol{\theta}_{3, E_{o}} \boldsymbol{\theta}_{2, o}}{\boldsymbol{\theta}_{3, E o} \boldsymbol{\theta}_{2, y}-\boldsymbol{\theta}_{2, E_{0}} \boldsymbol{\theta}_{3, y}} .
\end{gathered}
$$

Use that in the first two equations to replace $\varphi_{c y, s}$,

$$
\begin{array}{ll}
0=\theta_{4}+\theta_{4, s} \varphi_{s, s}+\theta_{4, o} \varphi_{c o, s}+\theta_{4, s} \varphi_{s, s}^{2} & +\theta_{4, s o} \varphi_{s, s} \varphi_{c o s} \\
0=\theta_{5}+\theta_{5, s} \varphi_{s, s}+\theta_{5, o} \varphi_{c o, s} & +\theta_{5, s o} \varphi_{s, s} \varphi_{c o, s}
\end{array}
$$


where

$\theta_{\mathbf{4}}=\theta_{1, y} \theta_{c y}$

$\theta_{\mathbf{4}, s}=\theta_{1, y} \theta_{c y, s}$

$\theta_{\mathbf{4}, o}=\theta_{1, y} \theta_{c y, c o}+\theta_{1, c o}$

$\theta_{4, s s}=\theta_{1, E y} \theta_{c y, s}$

$\theta_{4, s o}=\theta_{1, E y} \theta_{c y, c o}+\theta_{1, E o}$

$\theta_{5}=\theta_{2, y} \theta_{c y} \quad-1$

$\theta_{5, s}=\theta_{2, y} \theta_{c y, s}$

$\theta_{5, o}=\theta_{2, y} \theta_{c y, c o}+\theta_{2, o}$

$\theta_{5, s o}=\quad \theta_{2, E_{0}}$

Multiply the first of these two equations with $\theta_{5,50}$, the second with $-\theta_{4,50}$, add and solve for $\varphi_{c o, s}$,

$\varphi_{c o, s}=\theta_{c o}+\theta_{c o s} \varphi_{s, s}+\theta_{c o s s} \varphi_{s, s}^{2}$

where

$\theta_{c o}=\frac{\theta_{4, s o} \theta_{5}-\theta_{5, s o} \theta_{4}}{\theta_{5, s o} \theta_{4, o}-\theta_{4, s o} \theta_{5, o}}$

$\theta_{c o, s}=\frac{\theta_{\mathbf{4}, s o} \theta_{5, s}-\theta_{5, s o} \theta_{\mathbf{4}, s}}{\theta_{5, s o} \theta_{4, o}-\theta_{\mathbf{4}, s o} \theta_{5, o}}$

$\theta_{c o, s s}=\frac{-\theta_{5, s o} \theta_{4, s s}}{\theta_{5, s o} \theta_{4, o}-\theta_{4, s o} \theta_{5, o}}$

Use this to replace $\varphi_{c o, s}$ in either of the two equations of (172). We use the second equation and obtain

$0=\theta_{s}+\theta_{s, s} \varphi_{s, s}+\theta_{s, s s} \varphi_{s, s}^{2}+\theta_{s, s s s} \varphi_{s, s}^{3}$

where

$$
\begin{aligned}
& \theta_{s}=\theta_{5}+\theta_{5, o} \theta_{c o} \\
& \theta_{s, s}=\theta_{5, s}+\theta_{5, o} \theta_{c o s}+\theta_{5_{s o}} \theta_{c o} \\
& \theta_{s, s s}=\quad \theta_{5,0} \theta_{c o, s s}+\theta_{5, s o} \theta_{c o, s} \\
& \theta_{s, s s s}=\quad \theta_{5, s o} \theta_{c o, s s} \text {. }
\end{aligned}
$$


Equation (174) is a polynomial of third degree, which generally has three roots, $\varphi_{s, s, i}, i=1,2,3$, and for which closed-form solutions are available. If only one of these roots is stable, that is, less than 1 in absolute value, then this is the root we use. If there are more than one stable root, then an additional-state variable is needed as generally would be necessary to solve the system (172). If there are no stable roots, the system is explosive, and our baseline assumption that there is a stationary solution to the social planner's problem in the detrended variables is unjustified.

Experimentation with reasonable parameter choices has only delivered the case of a single stable root. We shall therefore concentrate on that case from here on. Thus, let $\varphi_{s, s}$ be that solution.

The other two key coefficients, $\varphi_{c o, s}$ and $\varphi_{c y, s}$, can now be found from equations (173) and (171).

For the remaining variables we have

$$
\begin{aligned}
& \varphi_{n, s}=\theta_{n, y} \varphi_{c y, s} \\
& \varphi_{y, s}=\ell_{f} \theta_{n, y} \varphi_{c y, s} \\
& \varphi_{k, s}=\theta_{k, y} \varphi_{c y, s}+\theta_{k, o} \varphi_{c o s} \\
& \varphi_{c w, s}=-\mu_{f} \theta_{n, y} \varphi_{c y, s} \\
& \varphi_{R, s}=\theta_{R, y} \varphi_{c y, s} \\
& \varphi_{a, s}=\theta_{a, y} \varphi_{c y, s}+\theta_{a, o} \varphi_{c o, s}+\theta_{a, E_{0}} \varphi_{s, s} \varphi_{c o, s},
\end{aligned}
$$

where $\varphi_{a, s}$ is the feedback coefficient for $\hat{a}_{t} /(1-\eta)$.

We summarize these feedback coefficients per

$$
\varphi_{\cdot s}=\left(\begin{array}{c}
\varphi_{s, s} \\
\varphi_{c y, s} \\
\varphi_{c o, s} \\
\varphi_{n, s} \\
\varphi_{y, s} \\
\varphi_{k, s} \\
\varphi_{w v, s} \\
\varphi_{R, s} \\
\varphi_{a, s}
\end{array} \mid .\right.
$$




\section{A.5.3 Step 3: Solving for the Coefficients on Exogenous Variables}

Solving for the exogenous variables is now a matter of solving a linear system of equations. In the equations (140) to (148), replace each endogenous variable dated $t$ with the feedback rule given in (159). The variable $\hat{s}_{t-1}$ stays as is. The variables dated $t+1$ show up in expectations, and are replaced with the feedback rules as, for example, in

$$
\begin{aligned}
E_{t}\left(c_{t+1,0}\right) & =E_{t}\left(\varphi_{c o, s} s_{t}+\varphi_{c o, \zeta} \zeta_{t+1}\right) \\
& =\boldsymbol{\varphi}_{c o, s} \varphi_{s, s} s_{t-1}+\left(\varphi_{c o, s} \varphi_{c o, b}+\varphi_{c o, b} N\right) \zeta_{t},
\end{aligned}
$$

where $\varphi_{s, s}$ and $\varphi_{c o, s}$ are now known, while we still seek to solve for $\varphi_{c o, r^{*}}$

The resulting system contains coefficients on the variable $s_{t-1}$, which we already know to hold from the previous calculations. Let $\varphi_{., \zeta}$ be the matrix of the to-be-solved-for feedback coefficients on the exogenous variables $\zeta$, given by

$$
\varphi_{\cdot, \zeta}=\left(\begin{array}{c}
\varphi_{s, b} \\
\varphi_{c y, \zeta} \\
\varphi_{c c, \zeta} \\
\varphi_{n, \zeta} \\
\varphi_{y, \zeta} \\
\varphi_{k, \zeta} \\
\varphi_{w, \zeta} \\
\varphi_{R, \zeta s} \\
\varphi_{a, \zeta}
\end{array}\right) .
$$

where $\varphi_{a, b}$ is the feedback coefficient for $\hat{a}_{t} /(1-\eta)$. Take the feedback coefficients $\varphi_{\text {., }}$ on $\hat{s}_{t-1}$ as given via the calculations above, and compare coefficients on the entries in $\zeta_{t}$. By carefully examining the system or, alternatively, exploiting the matrix algebra provided in Uhlig (1999), the remaining system can be written in the form

$V \operatorname{vec}(\varphi, r)=W$

for some matrices $V$ and $W$ and the columnwise vectorization $\operatorname{vec}(\varphi . f)$ of the matrix of coefficients $\varphi_{., 5}$. If $N=0$-that is, if the exogenous variables are i.i.d., then this can be written more conveniently as 
$(C \varphi \cdot, 6)=D$

In either case, one obtains a linear system in the entries of $\varphi_{., 6}$, which can be solved under the usual conditions for invertibility. We shall skip the tedious details on explicitely stating $V$ and $W$ or $C$ and $D$.

\section{A.5.4 Impulse responses}

Define the vector of endogenous variables

$$
\left.\psi_{t}=\mid \begin{array}{c}
\hat{s}_{t} \\
\hat{c}_{y, t} \\
\hat{c}_{o, t} \\
\hat{n}_{t} \\
\hat{y}_{t} \\
\hat{k}_{t} \\
\hat{w}_{t} \\
\hat{R}_{t} \\
\hat{a}_{t} /(1-\eta)
\end{array}\right) .
$$

With the above solution, one can now determine the effect of a shock $\varepsilon_{0}$ recursively per

$$
\begin{aligned}
& \zeta_{0}=\varepsilon_{0} \quad, \psi_{0}=\quad \varphi_{\cdot, \zeta} \zeta_{0} \\
& \zeta_{1}=N \zeta_{0}, \psi_{1}=\varphi_{\cdot s}\left(\psi_{0}\right)_{s}+\varphi_{\cdot, \zeta} \zeta_{1} \\
& \zeta_{2}=N \zeta_{1}, \psi_{2}=\varphi_{\cdot s}\left(\psi_{1}\right)_{s}+\varphi_{\cdot, \zeta} \zeta_{2}
\end{aligned}
$$

and so forth, where, for example, $\left(\psi_{0}\right)_{s}$ is the first entry (corresponding to $s_{0}$ ) of $\psi_{0}$. 


\section{Comment}

Henning Bohn, Department of Economics, University of California Santa Barbara, and CESifo

Bovenberg and Uhlig examine risk sharing in a stochastic overlappinggenerations model. Their approach is in many ways similar to my work on the same topic (Bohn 1998, 1999, 2001, 2002, 2003, 2005), and they reproduce several of my results. Like my papers, Bovenberg and Uhlig examine a stochastic two-period OG model, they log-linearize the constraints and first-order conditions, and they maximize an ex ante measure of welfare. There are three main differences: assumption about technology, assumption about intertemporal substitution, and modeling style. While Bovenberg and Uhlig assume AK production, I assume constant returns to scale. They assume an elasticity of intertemporal substitution greater than one $(1 / \eta>1)$, whereas I examine the entire range and focus on values below 1 . They start with a general model and then consider special cases, whereas I tend to focus on basic benchmark specifications and then generalize.

Bovenberg and Uhlig's main new finding concerns the optimal response to an unexpected permanent increase in longevity (finding number 3 in the abstract). In Bovenberg and Uhlig's model, the optimal response is to shift resources toward future consumption at the expense of current workers and current retirees. Capital investment increases, worker consumption declines, and retiree consumption per time unit declines so much that total retiree consumption declines. That is, retirees consume less over an unexpectedly long retirement period than they would have consumed otherwise. Bohn $(2001,2005)$ examines the same longevity shock in a similar two-period OG model. While worker consumption and retiree consumption per time unit also decline in my model, total retiree consumption increases and there is no significant change in capital investment. As I will explain, Bovenberg-Uhlig's result is due to endogenous labor combined with a high elasticity of intertemporal substitution. While I agree that endogenous labor has important 
ramifications, the high elasticity of intertemporal substitution seems counterfactual.

This comment has three parts. Part 1 provides an economic intuition and interpretation of the new result on longevity. Part 2 comments on other findings and how they relate to prior work. Part 3 raises some broader risk-sharing issues.

\section{A.1 The New Result on Longevity Shocks}

Bovenberg-Uhlig's most striking result is the decline in total retirement consumption in response to a longer retirement period. In their benchmark calibration, the elasticities of young-age $(=$ worker) and of total old-age ( $=$ retiree) consumption with respect to the longevity shock $\boldsymbol{\varpi}_{t}$ are:

$\varphi_{c y, \text { w }}=-0.93$ and $\varphi_{c o, \text { w }}=-0.39$,

respectively (see table 5.4, col.1). Because total old-age consumption is proportional to $\varpi_{t}$, the implied elasticity of old-age consumption per time unit is even more negative:

$\frac{d \ln \left(c_{o} / \varpi\right)}{d \ln (\varpi)}=\varphi_{c o . \varpi}-1=-1.39 \ll 0$.

The reduced consumption yields an increased capital stock, as confirmed by the positive elasticities with respect to capital growth; for example, $\varphi_{\text {growth, }}=1.25$ in col. 1 . $^{1}$

What explains these results? How general are they?

Bovenberg-Uhlig's explanation centers on increased marginal utility in old age that motivates higher returns on savings and makes it more attractive for the social planner to shift resources in the future (see section 4.6.7). This argument is difficult to follow because in the AK model, savings triggered by higher marginal utility in old age do not change the return on capital. In a model with decreasing returns to capital, the return would even decline.

A better intuition is to start with the observation that when the old live longer, a social planner will want to redistribute resources from young to old. For the young, the negative income effect increases labor supply. Because the shock is permanent, labor supply is also expected to increase in the future. In turn, more labor input increases the expected return to capital-not only in the AK model, but in any model where capital and labor are complements in production. As a result, the return to 
capital rises above the social planner's discount rate. The planner will want to implement an upward sloping optimal consumption path. An upward-sloping path must start at lower initial consumption to remain feasible. This line of argument matches the shape of the impulseresponse functions in figure 4.5 .

The key step in this argument is the link between higher labor supply and a permanently higher growth rate. In growth theory, this link is known as the scale effect (see Barro and Sala-i-Martin, 2004, ch. 4, for a textbook exposition.) The scale effect is a general feature of AK models, but unfortunately is inconsistent with empirical evidence (Jones 1995). It's regrettable that the scale effect is built into Bovenberg-Uhlig's anal$y$ sis and plays a role in their main result. ${ }^{2}$

Can the AK assumption be relaxed to avoid scale effects? And does the argument generalize to less persistent longevity shocks?

To examine these questions, consider a more general production function, though with somewhat simplified preferences. For production, suppose

$F\left(k_{t}, n_{t}\right)=y_{t}+(1-\bar{\delta}) k_{t}=k_{t}^{\theta_{k}} \cdot n_{t}^{1-\theta}+(1-\bar{\delta}) \cdot k_{t}$

with $0<\theta<1$ and $\theta_{k} \in(0,1)$. This includes Bovenberg-Uhlig's AKspecification for $\theta_{k}=1$ (their $f\left(n_{t}\right)=n_{t}^{1-\theta}$ case). For $\theta_{k} \in(\theta, 1)$, returns to scale are increasing, but returns to capital are decreasing. For $\theta_{k}=\theta$, one obtains constant returns to scale-Cobb-Douglas production.

For preferences, assume power utility with risk aversion parameter $\eta$ $>0$ and assume $v(n)=\bar{v} \cdot(1-n)^{1-\chi} /(1-\chi)$. These preferences are less flexible than Epstein-Zin utility; but unlike Bovenberg-Uhlig, I allow risk aversion to be above one. ${ }^{3}$ Let the longevity shock $\varpi_{\text {f }}$ follow a Markov process with AR-parameter $0 \leq \varphi_{\text {एक }} \leq 1$, which nests permanent and temporary shocks. For brevity in this comment, abstract from all other shocks, set $\bar{\delta}=1$, and assume zero population growth.

The optimal fluctuations of capital, consumption, and labor around a steady state follow a vector Markov process with state vector $\left(\breve{k}_{t}, \breve{\varpi}_{t}\right)$. (See the Appendix for all derivations and proofs.) The dynamics are fairly straightforward: if one substitutes out labor and young-age consumption, the dynamics of capital and old-age consumption are similar to a representative agent model with preference shocks. Longevity acts like a shock to marginal utility. Following Bohn (2003) and Campbell (1994), one can log-linearize the resource constraint and the first-order conditions analytically to obtain closed-form solutions for impulse- 
response coefficients (the $\varphi$-values). For AK-technology $\left(\theta_{k}=1\right)$, the process for capital has a unit root: $\varphi_{k, k}^{*}=1$. For $\theta_{k}<1$, the process for capital has a stable root $0<\varphi_{k, k}^{*}<1$ under modest parametric restrictions.

Most importantly, one can obtain closed-form solutions for the responses to longevity shocks. For capital and old-age consumption, the responses have the form:

$\varphi_{k, \mathrm{w}}=\frac{\left(\overline{c_{0}} / \bar{F}\right)\left[b_{1} \cdot \varphi_{\mathrm{w}, \mathrm{w}}-1\right]}{b_{0}-(\bar{k} / \bar{F})\left[b_{1} \cdot \varphi_{\mathrm{w}, \mathrm{t}}-1\right]}$ and $\varphi_{c o, \mathrm{\omega}}=1-\bar{\varphi}_{0}-\bar{\varphi}_{1} \cdot \varphi_{k, \mathrm{\omega}}$

where $b_{1}>1, b_{0}>(\bar{k} / \bar{F})\left(b_{1}-1\right)>0,0<\bar{\varphi}_{0}<1$, and $\bar{\varphi}_{1}>0$ are known functions of model parameters.

Bovenberg-Uhlig report that for all their numerical calibrations, total retiree consumption declines in response to longevity (that $\varphi_{c, \text {, }}<0$ ). The analytical solutions provide general conditions under which these results apply. For AK-technology and unit-root shocks, one can show:

Observation 1: If $\theta_{k}=1$ and $\varphi_{\mathrm{w}, \mathrm{\sigma}}=1, \varphi_{c, \mathrm{w}}<0$ holds if and only if $(1 / \eta)(\bar{k} /$ $\bar{F})>1+\left(\overline{c_{y}} / \bar{F}\right)\{\theta+[\bar{n} /(1-\bar{n})] /(1-\theta)\}$.

Because the expression on the right exceeds $1, \eta<(\bar{k} / \bar{F})<1$ is a necessary condition for $\varphi_{c o, \text { w }}<0$; and $\eta>(\bar{k} / \bar{F})$ is sufficient for $\varphi_{c o, \text {, }}>0$. A negative response of old-age consumption to longevity thus requires a sufficiently low risk-aversion parameter, or equivalently, an elasticity of intertemporal substitution exceeding $1 /(\bar{k} / \bar{F})>1$.

More generally, for all $\theta_{k} \leq 1$ and $\varphi_{w, \omega} \leq 1$, (1) implies:

Observation 2: The sign of $\varphi_{k, \mathrm{w}}$ is determined by $b_{1} \cdot \varphi_{w, w}-1$.

Because $b_{1}>1$, Bovenberg-Uhlig's assumption of $\varphi_{\varpi, \sigma}=1$ always ensures a positive capital response-regardless of other parameter choices. In general, a positive response of capital to longevity requires $\varphi_{\text {w, }}>\left(1 / b_{1}\right)$, that is, relatively persistent longevity shocks.

Observation 3: $\varphi_{c o, \omega}<0$ holds if and only if $\varphi_{k, \sigma}>\left(1-\bar{\varphi}_{0}\right) / \bar{\varphi}_{1}>0$; and $\varphi_{k, \text { w }}$ $>0$ implies $\varphi_{c o, \omega}<1$.

A positive response of capital to longevity $\left(\varphi_{k, w}>0\right)$ is thus sufficient for a decline in old-age consumption per time unit, but not sufficient for a decline in total old-age consumption. 
One can also show that a model with inelastic labor supply (no disutility) has solutions of the same form as (1), but with $b_{1}=1$. This implies:

Observation 4: With inelastic labor supply, permanent longevity shocks $\left(\varphi_{\omega, \omega}=1\right)$ imply $\varphi_{k, \omega}=0$ and $0<\varphi_{c o, \omega}<1$. That is, capital remains unchanged, total old-age consumption increases, and consumption per time unit declines.

Observation 4 reconciles the analysis here with the fixed-labor calibrations in Bohn $(2001,2005)$. With fixed labor, reduced worker consumption (one can show $\varphi_{c y, \sigma}<0$ ) does not trigger higher future labor supply and therefore no increase in the return to capital. This eliminates the planner's incentives to increase the capital stock and to reduce current consumption. Hence there is no investment response. Retiree consumption increases overall but decreases per time unit.

Bovenberg and Uhlig's significant contribution is to demonstrate the importance of endogenous labor supply in this context: Persistent longevity shocks imply a higher capital stock whenever the labor supply increases. For the consumption results, the necessity of a high elasticity of intertemporal substitution (EIS) is troubling, however. Empirical evidence suggests EIS values well below 1 (Ogaki and Reinhart 1998, Hall 1988). Unless one assumes a high EIS in addition to elastic labor supply, the results for consumption are qualitatively the same as in a fixed-labor model. Because the expressions in (1) are continuous in $\theta_{k}$, observations about AK also apply to production functions with $\theta_{k}$-values slightly less than 1.

Bovenberg and Uhlig argue that longevity is not valued unless one assumes $1 / \eta>1$. This argument is unconvincing. First, the value of life is irrelevant with exogenous longevity. Second, one could easily add terms involving " $\varpi_{t} \cdot$ (large weight)" to the utility function, which would leave marginal utility unchanged while making absolute utility increasing in longevity. The optimal allocation of consumption depends only on marginal utility, not on absolute utility.

In summary, the key assumption for Bovenberg and Uhlig's results on longevity is a high elasticity of intertemporal substitution. It makes the social planner willing to shift resources from current to future generations whenever the return on capital differs from the planner's rate of time preference. Though AK technology facilitates intertemporal shifts in resources, it is neither essential for the results nor appears necessary for tractability. 


\subsection{Some Known Results}

While I much appreciate Bovenberg-Uhlig's references to my work, links to the substantive results may be helpful to readers. In their abstract, Bovenberg and Uhlig highlight two findings:

1. The old typically bear a larger burden of the risk of productivity surprises, if old-age risk aversion is smaller than life risk aversion, and vice versa.

2. [The] consumption of the young and the old always move in the same direction, even for population growth shocks.

Finding (1) was first derived in Bohn (1998) in a very similar setting-a two-period stochastic OG model with Epstein-Zin preferences that allow for age-dependent risk aversion. The implications of age-dependent risk aversion are also discussed in Bohn (2003) in a habit model, and reviewed in Bohn (2005). The section on efficient allocations in Bohn (1998) concludes with:

Thus, an allocation that imposes more productivity risk on the young than on the old can be rationalized by preferences with age-increasing risk aversion. The old and the young should bear consumption risk in proportion to their risk tolerances. (Bohn 1998, p.18)

With regard to finding (2), Bohn (2005) states that

Efficient risk sharing has ... implications that are robust to differential risk aversion. Most importantly, everyone should be exposed to aggregate risk in the same direction. (Bohn 2005, p.10)

The context is a discussion of aggregate risks that include population growth shocks, longevity shocks, productivity shocks, and depreciation shocks.

Bohn (2001) examines a broader range of demographic shocks and derives conditions under which young and old should respond equally or unequally to demographic shocks. I distinguish not only permanent from temporary shocks, but also shocks revealed contemporaneously from anticipated demographic shocks. The timing matters because children are born about a generation before they start to work. Hence shocks to the labor force are known a generational period in advance. Contemporaneous shocks like Bovenberg-Uhlig's population growth shocks are better interpreted as shocks to the survival rate of children into adulthood. 
Regarding policy questions and decentralization, Bohn (2003) presents decentralization in terms of Generational Accounting, as adopted in Bovenberg-Uhlig's section 5. Regarding the "future work" proposed on p. 41, note that effects of pension reform on the equity premium are examined in Bohn (1997) and welfare implications of pension equity investments are examined in Bohn (1999). I find that the impact of plausible pension reforms on the equity premium would be non-zero, but very small, and that pension-equity investments can be Pareto improving if the gains are appropriately allocated.

Bovenberg and Uhlig do not log-linearize the model around its deterministic steady state - the common approach in macro-but around the mean values of a stochastic steady state. This is sketched out in sections 5.4.2 and Appendix 5.A.3. My interpretation of these sections is based on Bohn (1999), which used the same linearization method to examine the equity premium and the ramifications of social security investment policies. As explained therein, the issue is not only the equity premium, but also precautionary savings. The point of linearization is inessential for most other purposes; that is, a simpler linearization around the deterministic steady state would suffice (i.e., in terms of $\kappa_{3}, \kappa_{4}$, defined on p.27, the limiting case of zero variances).

The point of this section is not to question Bovenberg-Uhlig's originality, but to suggest that their contributions are different. They provide a flexible, all purpose specification that simultaneously includes many elements that in previous work were modeled one at a time. BovenbergUhlig's framework and programming algorithm are therefore ideal for finding interactions and linkages that were previously missed. Their analysis of longevity shocks exemplifies this strength: the possibility of declining total retiree consumption arises only in a generously parameterized model that allows the responses of several variables to interact and to reinforce each other.

In the same spirit, I suspect that Bovenberg-Uhlig's framework has rich and yet unexplored implications for the propagation of shocks. As explained in Bohn (2003), optimal risk sharing in an OG production economy has two parts. One part is static and involves optimal risk sharing between currently living cohorts. The other part is dynamic and involves the optimal propagation of shocks, which is the sharing of risk between current and future generations. Bovenberg-Uhlig's main technical innovation - the use of AK-technology - relates to capital accumulation, that is, to the model's propagation mechanism. The model should therefore deliver new insights about how risk is allocated over 
time. Static issues-ones dealing with current generations-deserve less attention, because analysis in this area is more likely to rediscover old results than to find new ones.

\subsection{Problems of Intergenerational Risk Sharing}

Bovenberg and Uhlig's paper has a clear normative focus: what is the optimal policy? Normative answers naturally trigger positive questions: how do empirically observed fiscal policies compare? Can we think of actual government policy as facilitating intergenerational risk sharing? The answers appear to be disappointing, unfortunately, perhaps even puzzling.

The main puzzle regards productivity risk, a major source of long-run uncertainty. Retirees tend to be less exposed to productivity risk than workers in market allocations (Bohn 2003, 2005). If old and young have equal relative risk aversion, they should be about equally exposed. Empirically plausible policies tend to protect retirees against risk, for example, by issuing safe debt and promising low-risk public pensions. Such policies magnify the underlying imbalances in risk exposures between retirees and workers. They are efficiency reducing unless retirees are substantially more risk averse than workers.

Can one argue that retirees are in fact more risk averse than workers? Regrettably, the OG model does not provide testable evidence. The risk aversion of the youngest cohort (or "life-risk aversion") is unobservable by construction. Economists usually infer preferences parameters from market actions-for example, the risk aversion of savers from portfolio choices, the elasticity of intertemporal substitution from savings-toreturn responses, and the labor supply elasticity from leisure-to-wage responses. This approach cannot be used for the risk aversion of the young, because they cannot trade before they are born. Otherwise, their risk aversion would presumably be reflected in their demand for insurance. Moreover, if future workers could trade-say, as children, or parents on their behalf-the policy problem would evaporate; policy would become irrelevant/neutral. In summary, lifetime risk aversion is a free parameter that makes the model difficult to test as a positive theory of policy.

Because of the unobserved-parameter problem, I have moved away from age-dependent Epstein-Zin preferences in Bohn (1998) to a habit formation in Bohn (2003). Habit formation implies higher risk aversion for the old than for the young, and it imposes more easily testable re- 
strictions. I suspect habit formation would be straightforward to include in Bovenberg-Uhlig's framework. I also believe the AK assumption could be relaxed without much loss of tractability. Both extensions would strengthen their paper's main contribution, which is to provide a well-integrated and flexible framework for doing welfare analysis in two-period stochastic OG models.

\section{References}

Barro, Robert, and Xavier Sala-i-Martin, 2004. Economic growth 2nd ed. Cambridge, MA: MIT Press.

Bohn, Henning, 1997. Social Security reform and financial markets. In Social Security reform, ed. Steven Sass and Robert Triest, 193-227. Boston: Federal Reserve Bank of Boston.

1998. Risk sharing in a stochastic overlapping generations model. Mimeo. Santa Barbara: University of California at Santa Barbara.

1999. Should the Social Security trust fund hold equities? An intergenerational welfare analysis. Review of Economic Dynamics 2: 666-97.

2001. Social Security and demographic uncertainty: The risk-sharing properties of alternative policies. In Risk aspects of investment based Social Security reform, ed. John Campbell and Martin Feldstein, 203-41. Chicago: University of Chicago Press.

2002. Retirement savings in an aging society: A case for innovative government debt management. In Ageing, Financial Markets and Monetary Policy, ed. Alan Auerbach and Heinz Herrman, 139-81. Berlin: Springer.

2003. Intergenerational risk sharing and fiscal policy. Mimeo. Santa Barbara: University of California at Santa Barbara.

2005. Forthcoming. Who bears what risk? An intergenerational perspective. In Restructuring retirement risks, ed. David Blitzstein, Olivia S. Mitchell, and Stephen P. Utkus. Oxford, UK: Oxford University Press.

Campbell, John. 1994. Inspecting the mechanism: An analytical approach to the stochastic growth model. Journal of Monetary Economics 33:463-506.

Hall, Robert. 1988. Intertemporal substitution in consumption. Journal of Political Economy 96:339-57.

Ogaki, Masao, and Carmen Reinhart. 1998. Measuring inter-temporal substitution: The role of durable goods. Journal of Political Economy 106 (5): 1078-98.

\section{Appendix: Derivations}

Under the assumptions stated in the text, the planning problem is to maximize

$\sum_{t=0}^{\infty} \omega^{t} E_{0}\left\{\omega\left[c_{t, y} \cdot \nu\left(n_{t}\right)\right]^{1-\eta}+\beta \cdot \varpi_{t}^{\eta} \cdot c_{t, o}^{1-\eta}\right\} /(1-\eta)$ subject to 
$c_{t, y}+c_{t, o}+k_{t+1}=F\left(k_{t}, n_{t}\right)=k_{t}^{\theta_{k}} \cdot n_{t}^{1-\theta}$

where $v(n)=\vec{v}(1-n)^{1-\chi} /(1-\chi), 0 \leq \chi<1$.

The first order conditions are

$$
\begin{aligned}
\omega \cdot c_{t, y}^{-\eta} \cdot v\left(n_{t}\right)^{1-\eta} & =\beta \cdot \varpi_{t}^{\eta} \cdot c_{t, o}^{-\eta} \\
F_{n}\left(k_{t} n_{t}\right) & =c_{t, y} \frac{-v^{\prime}\left(n_{t}\right)}{\nu\left(n_{t}\right)}=(1-\chi) \frac{c_{t, y}}{1-n_{t}} \\
c_{t, y}^{-\eta} \cdot v\left(n_{t}\right)^{1-\eta} & =\beta \cdot E_{t}\left[\varpi_{t+1}^{\eta} \cdot c_{t+1, o}^{-\eta} \cdot F_{k}\left(k_{t+1}, n_{t+1}\right)\right]
\end{aligned}
$$

Adopting Bovenberg-Uhlig's notation of inverted hats for logdeviations, log-linearizing (Ala-d) yields the following system of four equations in the four main variables (young consumption, old consumption, labor, and capital):

$$
\begin{aligned}
& \frac{\bar{c}_{y}}{\bar{F}} \check{c}_{t, y}+\frac{\bar{c}_{0}}{\bar{F}} \breve{c}_{t, o}+\frac{\bar{k}}{\bar{F}} \check{k}_{t+1}=\theta_{k} \cdot \check{k}_{t}+(1-\theta) \cdot \check{n}_{t} \\
& \check{c}_{t, y}-\kappa_{v n} \cdot \check{n}_{t}=\check{c}_{t, o}-\check{\varpi}_{t} \\
& \left(\theta+\frac{\bar{n}}{1-\bar{n}}\right) \check{n}_{t}=\theta_{k} \cdot \check{k}_{t}-\check{c}_{t, y}=\theta_{k} \cdot \check{k}_{t}-\left(\check{c}_{t, o}-\check{\varpi}_{t}\right)-\kappa_{v m} \cdot \check{n}_{t} \\
& \check{c}_{t, y}-\kappa_{\nu n} \cdot \check{n}_{t} \\
& =E_{t}\left[\left(\check{c}_{t+1, o}-\check{\varpi}_{t+1}\right)+\frac{1}{\eta}\left(1-\theta_{k}\right) \check{k}_{t+1}-\frac{1}{\eta}(1-\theta) \check{n}_{t+1}\right],
\end{aligned}
$$

where $\kappa_{v n}=[1-(1 / \eta)](1-\chi)[\bar{n} /(1-\bar{n})]$. If one substitutes out labor and young-age consumption, the system reduces to two equations for capital and for old-age consumption:

$$
\begin{aligned}
& \left(\kappa_{c}+\kappa_{n}\right) \check{c}_{t, 0}+\left(1-\kappa_{c}\right) \check{k}_{t+1}=\left(1+\kappa_{n}\right) \theta_{k} \cdot \check{k}_{t}+\left(\kappa_{c y}+\kappa_{n}\right) \check{\varpi}_{t} \\
& \check{c}_{t, 0}-\check{\varpi}_{t}=E_{t}\left[\left(1+\frac{1}{\eta} \kappa_{\theta}\right)\left(\check{c}_{t+1, o}-\check{\varpi}_{t+1}\right)+\frac{1}{\eta}\left(1-\theta_{k}-\theta_{k} \cdot \kappa_{\theta}\right) \check{k}_{t+1}\right],
\end{aligned}
$$

where $\boldsymbol{\kappa}_{c y}=\bar{c}_{y} / \bar{F}>0, \boldsymbol{\kappa}_{c}=\left(\bar{c}_{y}+\bar{c}_{o}\right) / \bar{F}>\boldsymbol{\kappa}_{c y}>0, \boldsymbol{\kappa}_{\theta}=(1-\theta) /\left\{\theta+\boldsymbol{\kappa}_{\nu n}+\right.$ $[\bar{n} /(1-\bar{n})]\}$, and $\kappa_{n}=1-\theta-\kappa_{c y} \kappa_{v n} /\left\{\theta+\kappa_{v n}+[\bar{n} /(1-\bar{n})]\right\}$. Also define $\left(\kappa_{\mathrm{co}}\right.$ $=\bar{c}_{o} / \bar{F}=\kappa_{c}-\kappa_{c y}>0$.

Note that the same dynamics apply in a model with fixed labor supply (no disutility: $v=\bar{v}$ ), provided one replaces (A2c) by $\breve{n}_{t} \equiv 0$ and imposes $\kappa_{n}=\kappa_{\theta}=0$ in (A3).

The rational-expectations solution for $\left(\breve{k}_{\mathrm{t}+1}, \breve{c}_{t, 0}\right)$ follows a Markov pro- 
cess with state vector $\left(\breve{k}_{t}, \breve{\varpi}_{t}\right)$. Let $\left(\varphi_{k, k}, \varphi_{c o, k}\right)$ and $\left(\varphi_{k, \mathrm{w}}, \varphi_{c 0, \mathrm{w}}\right)$ denote the elasticities of the endogenous variables with respect to capital and longevity, respectively. Matching coefficients in (A3), one obtains a pair of equations for each pair of elasticities:

$\left(\kappa_{c}+\kappa_{n}\right) \varphi_{c o, k}+\left(1-\kappa_{c}\right) \varphi_{k, k}=\left(1+\kappa_{n}\right) \theta_{k}$

$\varphi_{c o, k}=\left[\left(1+\frac{1}{\eta} \kappa_{\theta}\right) \varphi_{c o, k}+\frac{1}{\eta}\left(1-\theta_{k}-\theta_{k} \cdot \kappa_{\theta}\right)\right] \varphi_{k, k}$

that characterize the propagation of shocks to capital; and

$$
\begin{aligned}
\left(\kappa_{c}+\kappa_{n}\right) \varphi_{c o, \mathrm{w}}+\left(1-\kappa_{c}\right) \varphi_{k, \mathrm{\sigma}}=\left(\kappa_{c y}+\kappa_{n}\right) & \\
\varphi_{c o, \mathrm{\sigma}}-1= & {\left[\left(1+\frac{1}{\eta} \kappa_{\theta}\right) \varphi_{c o, k}+\frac{1}{\eta}\left(1-\theta_{k}-\theta_{k} \cdot \kappa_{\theta}\right)\right] \varphi_{k, \mathrm{\sigma}} } \\
& +\left(1+\frac{1}{\eta} \kappa_{\theta}\right) \varphi_{\varpi, \mathrm{\sigma}} \cdot\left(\varphi_{c o, \mathrm{\sigma}}-1\right)
\end{aligned}
$$

characterize the responses to longevity shocks.

To simplify the algebra, define a more compressed notation for (A4):

$$
\begin{aligned}
a_{1} \varphi_{c o, k}+a_{2} \varphi_{k, k} & =a_{3} \\
\varphi_{c o, k} & =\left(b_{1} \varphi_{c o, k}+b_{2}\right) \varphi_{k, k}
\end{aligned}
$$

where $a_{1}=\kappa_{c}+\kappa_{n^{\prime}} a_{2}=1-\kappa_{c^{\prime}} a_{3}=\left(1+\kappa_{n}\right) \theta_{k^{\prime}} b_{1}=\left[1+(1 / \eta) \kappa_{\theta}\right], b_{2}=$ $(1 / \eta)\left(1-\theta_{k}-\theta_{k} \cdot \kappa_{\theta}\right)$.

Equations (A6) yield a quadratic equation for $\varphi_{k, k}$ :

$P\left(\varphi_{k, k}\right) \equiv b_{1} a_{2} \varphi_{k, k}^{2}-\left(a_{2}+b_{1} a_{3}+b_{2} a_{1}\right) \varphi_{k, k}+a_{3}=0$.

It is straightforward to verify that for $0<\theta_{k}<1$, this equation has a unique stable (and positive) root, denoted $\varphi_{k, k}^{*} \in(0,1)$, provided $\theta+\bar{n} /(1-\bar{n})+$ $\kappa_{v n}>0 .\left(P[0]=a_{3}>0\right.$ and $P[\infty]=\infty$ are obvious. The main step is to verify $P[1]<0$ for $0<\theta_{k}<1$, which implies one root in $[0,1]$, the other in [1, $\infty]$.)

For $\theta_{k}=1$, (A6) also applies and has solutions $\varphi_{k, k}^{*}=\varphi_{c o, k}^{*}=1$. (One can verify $P[1]=0$. Bovenberg and Uhlig instead transform the model into stationary "capital-ratio" variables; this yields the same results as shown below.)

Given $\varphi_{k, k}^{*}$, one obtains $\varphi_{c o, k}^{*}=\left(a_{3}-a_{2} \varphi_{k, k}^{*}\right) / a_{1}$ for consumption. Note that $\left(\varphi_{k, k}^{*}, \varphi_{c o, k}^{*}\right)$ are known functions of the model parameters. 
Continuing with general $\theta_{k} \leq 1$, (A5) can be written more compactly as

$$
\begin{aligned}
a_{1}\left(\varphi_{c o, \sigma}-1\right)+a_{2} \varphi_{k, \sigma} & =\kappa_{c y}-\kappa_{c}=\kappa_{c o}<0 \\
\varphi_{c o, \sigma}-1 & =\left(b_{1} \varphi_{c o, k}^{*}+b_{2}\right) \varphi_{k, \sigma}+b_{1} \varphi_{\varpi, \sigma}\left(\varphi_{c o, \omega}-1\right),
\end{aligned}
$$

which implies

$$
\begin{aligned}
& \varphi_{k, \omega}=\frac{\kappa_{c o}\left(b_{1} \cdot \varphi_{\omega, \omega}-1\right)}{b_{0}-a_{2}\left(b_{1} \cdot \varphi_{\omega, \omega}-1\right)}, \text { and } \\
& \varphi_{c o, \omega}=1-\frac{\kappa_{c o}}{a_{1}}-\frac{a_{2}}{a_{1}} \varphi_{k, \omega}=1-\bar{\varphi}_{0}-\bar{\varphi}_{1} \cdot \varphi_{k, \omega},
\end{aligned}
$$

where $b_{0}=a_{1}\left(b_{1} \varphi_{c 0, k}^{*}+b_{2}\right)=b_{1}\left(a_{3}-a_{2} \varphi_{k, k}^{*}\right)+a_{1} b_{2}, \bar{\varphi}_{0}=\kappa_{c o} / a_{1}$, and $\bar{\varphi}_{1}=a_{2} /$ $a_{1}$. Equations (A8) and (A9) match equation (1) in the text.

Note that $a_{1}=\kappa_{c}+\left(1-\theta-\kappa_{c y} \kappa_{v n}\right) /\left\{\theta+\kappa_{v n}+[\bar{n} /(1-\bar{n})]\right\}=\kappa_{\theta}+\kappa_{c o}+$ $\kappa_{c y}\{\theta+[\bar{n} /(1-\bar{n})]\} /\left\{\theta+\kappa_{v n}+[\bar{n} /(1-\bar{n})]\right\}>\kappa_{\theta}+\kappa_{c o}>0$ and $\kappa_{\theta}>0$, provided $\theta+\bar{n} /(1-\bar{n})+\kappa_{v n}>0$. Under this condition, $\bar{\varphi}_{0}, \bar{\varphi}_{1}>0$ and $\bar{\varphi}_{0}<1$, which implies Observation 2 . The denominator in (A8) is declining in $\varphi_{w, \sigma}$. Hence, a positive sign of $d_{1} \equiv b_{0}-a_{2}\left(b_{1}-1\right)$ ensures a positive denominator for all $\varphi_{\omega, \omega}$. Writing

$d_{1}=a_{2}\left(1-b_{1} \varphi_{k, k}^{*}\right)+b_{1}\left(a_{3}-a_{2}\right)+b_{2} a_{1}=a_{3} / \varphi_{k, k}^{*}-a_{2} b_{1}$

one finds $d_{1}>0 \Leftrightarrow a_{3} / \varphi_{k, k}^{*}>a_{2} b_{1} \Leftrightarrow \varphi_{k, k}^{*}<a_{3} /\left(a_{2} b_{1}\right)$. The latter is true because the two roots of $P\left(\varphi_{k, k}\right)=0$ multiply to $a_{3} /\left(a_{2} b_{1}\right)$ and the greater root exceeds 1. Thus, (A8) has a positive denominator. Observation 3 follows because $\kappa_{c o}>0$. For Observation 4 , note that in a fixed-labor model, $\kappa_{\theta}=$ 0 implies $b_{1}=1$ and hence $\varphi_{k, \mathrm{w}}=0$ for $\varphi_{\mathrm{w}, \mathrm{w}}=1$.

For Observation 1, one requires a more detailed analysis of the condition

$\varphi_{k, \mathrm{w}}>\left(1-\bar{\varphi}_{0}\right) / \bar{\varphi}_{1}=\left(\kappa_{n}+\kappa_{c y}\right) /\left(1-\kappa_{c}\right)$

that ensures $\varphi_{c, \sigma}<0$. For simplicity, I focus on the AK-production case:

Lemma A1: Assume $\theta_{k}=1$ and $\varphi_{\omega, \omega}=1$. Then $\varphi_{c o, \omega}<0$ holds if and only if $(1 / \eta)(\bar{k} / \bar{F})>1+\kappa_{c y}\{\theta+[\bar{n} /(1-\bar{n})]\} /(1-\theta)>1$.

Proof: $\theta_{k}=1$ and $\varphi_{\mathrm{w}, \mathrm{\omega}}=1$ imply $b_{2}=-(1 / \eta) \kappa_{\theta}=1-b_{1}$ and $d_{1}=a_{1}\left(b_{1}+b_{2}\right)$ $-a_{2}\left(b_{1}-1\right)=a_{1}-a_{2}\left(b_{1}-1\right)$. 
From (A9), $\varphi_{c o, \text { w }}<0$ is equivalent to $a_{1}-\kappa_{c o}-a_{2} \cdot \varphi_{k, \mathrm{w}}<0$, where $\varphi_{k, \mathrm{w}}=\kappa_{c o}\left(b_{1}\right.$ $-1) / d_{1}$. Hence

$$
\begin{aligned}
& \varphi_{c o, \mathrm{w}}<0 \Leftrightarrow a_{1} d_{1}<\kappa_{c o}\left[d_{1}+a_{2}\left(b_{1}-1\right)\right]=b_{0} \kappa_{c o}=a_{1} \kappa_{c o} \Leftrightarrow d_{1}<\kappa_{c o} \\
& \Leftrightarrow d_{1}=a_{1}-a_{2}\left(b_{1}-1\right)=\left(\kappa_{c}+\kappa_{n}\right)-\left(1-\kappa_{c}\right) \frac{1}{\eta} \kappa_{\theta}<\kappa_{c o} \\
& \Leftrightarrow \kappa_{c y}+\kappa_{n}<\left(1-\kappa_{c}\right) \frac{1}{\eta} \kappa_{\theta} \\
& \Leftrightarrow \kappa_{c y}+\frac{1-\theta-\kappa_{c y} \kappa_{v n}}{\theta+\kappa_{v n}+\frac{\bar{n}}{1-\bar{n}}}<\frac{1}{\eta}\left(1-\kappa_{c}\right) \frac{1-\theta}{\theta+\kappa_{v n}+\frac{\bar{n}}{1-\bar{n}}} \\
& \Leftrightarrow \kappa_{c y} \frac{\theta+\frac{\bar{n}}{1-\bar{n}}}{\theta+\kappa_{v n}+\frac{\bar{n}}{1-\bar{n}}}<\left[\frac{1}{\eta}\left(1-\kappa_{c}\right)-1\right] \frac{1-\theta}{\theta+\kappa_{v n}+\frac{\bar{n}}{1-\bar{n}}} \\
& \Leftrightarrow \kappa_{c y} \frac{\theta+\frac{\bar{n}}{1-\bar{n}}}{1-\theta}<\frac{1}{\eta}\left(1-\kappa_{c}\right)-1 \Leftrightarrow \frac{1}{\eta}\left(1-\kappa_{c}\right)>\kappa_{c y} \frac{\theta+\frac{\bar{n}}{1-\bar{n}}}{1-\theta} \\
& +1>1 \text {, where } 1-\kappa_{c}=\bar{k} / \bar{F} \text {. } \\
& \text { QED. }
\end{aligned}
$$

Corollary: A necessary condition for $\varphi_{c o, \sigma}<0$ is a $\eta<1-\kappa_{c}<1$. That is, the risk-aversion parameter is much less than the gross investment share in output, which is less than 1. Equivalently, the elasticity of intertemporal substitution must exceed $1 /\left(1-\kappa_{c}\right)>1 .^{5}$

Finally, one may verify that Bovenberg-Uhlig's transformed variables $\left(\breve{c}_{t, y}-\breve{k}_{t}, \breve{k}_{t+1}-\breve{k}_{t}\right)$ yield the same results as the above derivations. Equations (A3) with $\theta_{k}=1$ imply the transformed system

$$
\begin{aligned}
& \left(\kappa_{c}+\kappa_{n}\right)\left(\breve{c}_{t, o}-\breve{k}_{t}\right)+\left(1-\kappa_{c}\right)\left(\breve{k}_{t+1}-\breve{k}_{t}\right)=\left(\kappa_{c y}+\kappa_{n}\right) \breve{\varpi}_{t} \\
& \breve{c}_{t, o}-\breve{k}_{t}-\breve{\varpi}_{t}=E_{t}\left[\left(1+\frac{1}{\eta} \kappa_{\theta}\right)\left(\breve{c}_{t+1, o}-\breve{k}_{t+1}-\breve{\varpi}_{t+1}\right)\right]+\left(\breve{k}_{t+1}-\breve{k}_{t}\right)
\end{aligned}
$$

Because the state vector is the single variable $\breve{\varpi}_{t^{\prime}}$ the solutions for $\left(\breve{c}_{t, 0}-\right.$ $\breve{k}_{t}, \breve{k}_{t+1}-\breve{k}_{t}$ ) are linear functions of $\breve{\varpi}_{t}$. Denote the slope coefficients by $\left(\varphi_{c 0-k, w^{\prime}} \varphi_{\text {growth, },}\right)$. From (A10): 
$\left(\kappa_{c}+\kappa_{n}\right) \varphi_{c o-k, \omega}+\left(1-\kappa_{c}\right) \varphi_{\text {growth, } w}=\left(\kappa_{c y}+\kappa_{n}\right)$

$\varphi_{c o-k, \omega}-1=\left(1+\frac{1}{\eta} \kappa_{\theta}\right)\left(\varphi_{c o-k, \omega}-1\right) \varphi_{\varpi \omega}+\varphi_{\text {growth, } \omega}$

which yields $\varphi_{\text {growth,w }}=\kappa_{c o}\left\{\varphi_{\text {एक् }}\left[1+(1 / \eta) \kappa_{\theta}\right]-1\right\} /\left(\kappa_{c}+\kappa_{n}-\left(1-\kappa_{c}\right)\left\{\varphi_{\text {ww }}[1\right.\right.$ $\left.\left.\left.+(1 / \eta) \kappa_{\theta}\right]-1\right\}\right), \varphi_{c 0-k, w}=\left(1-\kappa_{c 0}\right) /\left(\kappa_{c}+\kappa_{n}\right)-\left(1-\kappa_{c}\right) /\left(\kappa_{c}+\kappa_{n}\right) \varphi_{\text {growth,w }}$. For $\theta_{k}=1$, the denominator in (A8) reduces to the same expression as the denominator in $\varphi_{\text {growth, }}$ (see proof of Lemma A1). Comparing to (A8-9), one finds $\varphi_{\text {growth, } \mathrm{w}}=\varphi_{k, \mathrm{w}}$ and $\varphi_{c o-k, \mathrm{\omega}}=\varphi_{c o, \mathrm{~T}}$.

\section{Notes}

1. For visual reinforcement, the impulse responses in figure 5.1 show that growth rises persistently and that the capital stock grows exponentially relative to the baseline (linearly in logs). According to figure 5.1, future consumption/capital ratios also decline, but because capital grows faster, future generations-beyond a transition period--will enjoy higher and higher consumption levels in old age and in young age. Other columns in table 5.4 show even greater negative consumption elasticities.

2. Bovenberg-Uhlig select the "benchmark parametrization" so there is zero growth in the initial steady state (see first paragraph of sec. 5.6.1), perhaps to avoid scale effects. This does not solve the problem, however, because longevity shocks trigger permanent deviations of growth form the steady state; that is, exponentially growing deviations in levels that introduce non-zero growth.

3. As explained below, I am not persuaded by their argument for $\eta<1$.

4. The term rationalized actually hints at a problem: the risk aversion of the young-which Bovenberg and Uhlig call life risk aversion-is unobservable because individuals entering the economy are, by construction, unable to express their risk preferences through prior market actions. Because of this problem, and to avoid non-expected utility, my more recent work presents age-dependent risk aversion in terms of a habit model (Bohn 2003).

5. Bovenberg-Uhlig report $\varphi_{c, t, s}<0$ in table 5.4 even though $\eta=0.5$ exceeds $\bar{k} / \bar{F} \approx 0.4$. This suggests that more complicated conditions apply for Epstein-Zin preferences. 


\section{Comment}

Philippe Weil, ECARES, Free University of Brussels, and NBER

\section{Introduction}

\subsection{General Comments}

The paper by Bovenberg and Uhlig represents an ambitious effort. Its objective is to provide a quantitative characterization of optimal intergenerational risk sharing in a world in which almost everything is random (productivity, demography, longevity), and in which investment is the engine of long-run growth (AK model). This analysis is carried out in a log-linear framework, so that it is possible to precisely understand the role of preferences and technology on the shape of the optimal policy mix.

\subsection{General Caveat}

One might wonder, at the outset, whether it makes sense to characterize in such detail the optimal intergenerational redistribution while neglecting the very real possibility that public schemes at least partly crowd out, or substitute for, risk sharing within the family. The point is not that one must jettison the overlapping-generations (OLG) model in favor of a Ricardian alternative, but instead that one should at least acknowledge that the risks affecting a cohort are, to some extent, shared without public intervention inside altruistic families (e.g., the risk of being born disabled, or during a recession). So it should be clearly stated that the view taken by this paper, and others like it, is no less extreme than that of 100-percent Ricardian models. The latter assume all intergenerational risk sharing is useless, the former that all intergenerational risk sharing matters. The truth is obviously somewhere in between. Un- 
fortunately, we have little empirical evidence as to where we stand, quantitatively, between these two extremes. This turns what should be an empirical debate into an ideological argument, with the unfortunate result that papers in the intergenerational risk-sharing literature usually preach to those who are already converted.

\section{Contributions of the paper}

\subsection{Related Literature}

What does this paper achieve relative to previous literature?

- Relative to Bohn (2004): Bovenberg and Uhlig use Epstein-Zin-Weil preferences, but Bohn (2004) already separated risk aversion from intertemporal substitution using preferences exhibiting habit formation. Bohn also examined the effect of age-dependent risk aversion, and of labor-leisure choices.

- Relative to Krueger and Kubler: Bovenberg and Uhlig go beyond simple pay-as-you-go systems, and compute optimal mix between defined benefits and defined contributions, and funded/unfunded systems.

- Relative to Barbie, Hagedorn, and Kaul (2005): Bovenberg and Uhlig deal with ex ante efficiency. This is similar to Bohn (2003).

- Relative to all: Bovenberg and Uhlig AK model. This enables them to introduce a feedback from the work effort of young onto capital accumulation and long-run growth.

\subsection{Main Results}

The paper shows that in an ex ante optimal allocation:

1. The consumptions of young and old move together.

2. The old bear a larger share of macro risk than the young if they are relatively more risk averse.

3. Permanent aging of the population requires lower total consumption of the old (and more work by the young, in order to increase savings, and thus growth, in this AK model).

Results 1 and 2 are not surprising, as they follow from standard considerations in the theory of efficient risk sharing. Result 3 is less intuitive 
(but see below for comments on the formalization of longevity adopted in the model).

\section{Intuition}

Competitive equilibria in OLG models are generically not ex ante Pareto-efficient, even in economies that are dynamically efficient. The one notable exception, studied extensively in Blanchard and Weil (2001) and Bohn (2003) is a simple Diamond model with logarithmic utility, Cobb-Douglas production function, and full capital depreciation. I will heavily draw on Blanchard and Weil (2001) to outline this model, as it neatly illustrates the issues at hand.

\subsection{Consumption}

Suppose a typical member of generation $t$ faces the problem of allocating an initial labor income $W_{t}$ (which is random, but realized at birth) between young-and old-age consumption $C_{1, t}$ and $C_{2, t+1}$. She supplies one unit of labor when young at the going wage rate $W_{t}$, and retires when old. She maximizes

$(1-\beta) \ln C_{1, t}+\beta_{t} \ln C_{2, t+1}$

subject to the budget constraints

$\mathrm{C}_{1, t}+S_{t}=\mathrm{W}_{t}$

$$
C_{2, t+1}=R_{t+1} S_{t}
$$

where $S_{t}$ is savings and $R_{t+1}$ denotes the gross interest rate between dates $t$ and $t+1$. It is straightforward to show that the optimal consumption of the young and old who are alive at time $t$ is

$C_{1, \varepsilon}=\beta W_{t}$

$C_{2, t}=R_{t} \beta W_{t-1}$

so that

$$
\frac{C_{2, t}}{C_{1, t}}=\frac{R_{t} W_{t-1}}{\mathrm{~W}_{t}} \text {. }
$$




\subsection{Production}

Suppose output at $t$ is

$Y_{t}=A_{t} K_{t}^{\alpha} N_{t}^{1-\alpha}$,

where $A_{t}$ is a productivity shock realized one instant before the birth of generation $t$, and $K$ and $N$ denote the capital and labor inputs. Capital fully depreciates in production, and firms maximize profits under perfect competition.

\subsection{Competitive Equilibrium}

Suppose population is constant and normalized to 1 , so that $N_{t}=1$ for all $t$. In this economy, with full capital depreciation, clearing of the goods market requires that the capital stock at the beginning of period $t+1$ equal the savings of the young at $t$ :

$K_{t+1}=S_{t}$.

Using the first-order conditions for utility and profit maximization, this implies that

$K_{t+1}=\beta(1-\alpha) A_{t} K_{t}^{\alpha}$,

which is the standard (stochastic) difference equation characterizing the dynamics of the capital stock in the Diamond model.

Since $R_{t}=\alpha A_{t} K_{t}^{\alpha-1}$, this can be shown to imply that

$\frac{R_{t} W_{t-1}}{W_{t}}=\frac{\alpha}{(1-\alpha) \beta} \equiv \theta$.

Therefore:

$\frac{C_{2, t}}{C_{1, t}}=\theta$

so that the consumption of the young and of the old are perfectly correlated in the competitive equilibrium.

\subsection{Optimality}

One can show that, in this economy, the unconditional expectation of the logarithm of the gross marginal product of capital is $\ln \theta$. Thanks to Zilcha 
(1990), we know that in this example without population growth, the condition $\ln \theta>0$, that is, $\theta>1$, entails dynamic efficiency.

It turns out that the condition $\theta>1$ also entails ex ante Pareto optimality, since the competitive allocation then maximizes the social welfare function

$\left.t \sum_{s=0}^{\infty}(1+\theta)^{-s}\left[(1-\beta) \ln C_{1, t+s}+\beta \ln C_{2, t+s+1}\right)\right]$.

\subsection{Generalization}

The perfect correlation of the consumption of old and young is lost when one departs from this simple/Cobb-Douglas model. But it is what is required, as Bovenberg and Uhlig show, to guarantee ex ante optimality-whence the role of Pareto-improving intergenerational risksharing public policies (result 1). Were we to allow for different degrees of risk aversion when young and old, we would obviously recover result 2, namely that optimal intergenerational risk-bearing requires that the less risk averse bear more risk.

\section{Longevity}

\subsection{Optimal Suicide}

A fascinating issue arises in the paper as to the preference for longevity. The easiest way to illustrate it is to consider the following simple example.

Suppose that I have one unit of good to spend, and that I can choose either to eat it all now and die right afterward, or to eat $1 / 2$ of it now and $1 / 2$ of it tomorrow.

Ignoring time discounting, and with a utility function $q(\cdot)$ over goods, the former strategy yields utility $q(1)$, the latter $2 q(1 / 2)$. As a result, it is optimal to live fast and furious rather than slow and easy (i.e., to commit suicide young) if and only if

$q(1)>2 q(1 / 2)$,

or, more generally, if the derivative of the function $\omega q(1 / \omega)$ with respect to $\omega$ is negative.

To make sure this does not occur, the authors impose the restriction that $q(z)-z q^{\prime}(z)>0$ for all $z$. This is a bizarre restriction, as it rules out, 
for instance, all isoelastic functions $q(\cdot)$ at least as concave as logarithmic utility.

\subsection{The Benefits of Living}

The reason for this absurd parametric restriction is that the formalization of preferences in the paper is incomplete in a crucial dimension that is not orthogonal to the issue of longevity: the authors assume that the old do not value leisure. As a result, it is neither pathological nor surprising that agents may sometimes want to die young.

To avoid optimal suicide without imposing an ad hoc assumption on the utility function, it suffices to assume that living longer is in itself pleasurable (as we implicitly do when we assume that leisure is pleasurable per se). Call $\lambda>0$ the utility value of an extra period of time (net of the extra hours spent in the gym to increase longevity). Then, living fast and furious provides utility $q(1)+\lambda$, while slow and easy yields utility $2 q(1 / 2)+2 \lambda$. As a result, it is optimal to die old provided that $2 q(1 / 2)+$ $2 \lambda>q(1)+\lambda$, that is, when

$\lambda>q(1)-2 q(1 / 2)$.

To ensure that this condition is satisfied, there is no need to impose anymore that the function $\omega q(1 / \omega)$ be decreasing in $\omega$. All that is required is that $\lambda$ be big enough.

I conclude from this simple calculation that the paper is missing a more careful consideration of the costs and benefits of longevity. This is, of course, the message of Hamermesh and Soss (1974), who quote Schopenhauer's On Suicide:

... as soon as the terrors of life reach the point at which they outweigh the terrors of death, a man will put an end to his life.

Until the authors present a more complete theory of endogenous longevity, their results on the appropriate policy response to longevity shocks should thus be taken with a grain of salt.

\section{Conclusion}

Before I wrap up this discussion, I would like to reflect briefly on the ex ante efficiency criterion used in the paper. The debate between advocates of ex ante and interim efficiency is not an academic argument but an important policy debate. It rests on two main considerations: 
- The intergenerational transfers required by the more demanding ex ante efficiency concept are in most cases not implementable in a democracy. Hence there is no guarantee (in contrast with policies that implement interim efficiency) that future generations will respect the social intergenerational insurance contract signed today to enforce ex ante efficiency.

- By contrast, the less ambitious interim optimal allocations can be implemented by sophisticated, state-contingent, Ponzi schemes in which agents take part voluntarily (Blanchard and Weil, 2001; Barbie et al., 2003).

To sum up, this paper is a technical tour de force. Despite all its ingredients, it remains tractable, which is a real feat. It suffers from an overly terse formalization of the preference for longevity and lacks a discussion of the appropriateness of the ex ante efficiency criterion. But it is fascinating.

\section{References}

Blanchard, Olivier, and Philippe Weil. 2001. Dynamic efficlency, the risk less rate, and debt Ponzi games under uncertainty. Advances in Macroeconomics 1(2): 1031-31. Berkeley, CA: Berkeley Electronic Press.

Bohn, Henning. 2004. Intergenerational risk sharing and fiscal policy. 2004 Meeting Papers no. 22. New York: Society for Economic Dynamics.

Hamermesh, Daniel S., and Neal M. Soss. 1974. An economic theory of suicide. Journal of Political Economy 82(1): 83-98.

Zilcha, Itzhak. 1990. Dynamic efficiency in overlapping generations models with stochastic production. Journal of Economic Theory 52(2): 364-79. 